DOI 10.4171/JEMS/343

Ngaiming Mok

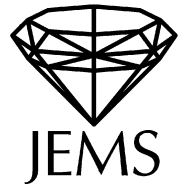

\title{
Extension of germs of holomorphic isometries up to normalizing constants with respect to the Bergman metric
}

Received August 24, 2010 and in revised form September 7, 2011

\begin{abstract}
We study the extension problem for germs of holomorphic isometries $f:\left(D ; x_{0}\right) \rightarrow$ $\left(\Omega ; f\left(x_{0}\right)\right)$ up to normalizing constants between bounded domains in Euclidean spaces equipped with the Bergman metrics $d s_{D}^{2}$ on $D$ and $d s_{\Omega}^{2}$ on $\Omega$. Our main focus is on boundary extension for pairs of bounded domains $(D, \Omega)$ such that the Bergman kernel $K_{D}(z, w)$ extends meromorphically in $(z, \bar{w})$ to a neighborhood of $\bar{D} \times D$, and such that the analogous statement holds true for the Bergman kernel $K_{\Omega}(\zeta, \xi)$ on $\Omega$. Assuming that $\left(D ; d s_{D}^{2}\right)$ and $\left(\Omega ; d s_{\Omega}^{2}\right)$ are complete Kähler manifolds, we prove that the germ of $f$ extends to a proper holomorphic isometric embedding such that $\operatorname{Graph}(f)$ extends to a complex-analytic subvariety on some neigborhood of $\bar{D} \times \bar{\Omega}$. In the event that the Bergman kernel $K_{D}(z, w)$ extends to a rational function of $(z, \bar{w})$ and the analogue holds true for the Bergman kernel $K_{\Omega}(\zeta, \xi)$, we show that $\operatorname{Graph}(f)$ extends to an affine-algebraic variety. Our results apply in particular to pairs $(D, \Omega)$ of bounded symmetric domains in their Harish-Chandra realizations. When $D$ is the complex unit ball $B^{n}$ of dimension $n \geq 2$, we obtain a new rigidity result which guarantees the total geodesy of the map under certain conditions. On the other hand, we construct examples of holomorphic isometries of the unit disk into polydisks which are not totally geodesic, answering in the negative a conjecture of Clozel-Ullmo's.
\end{abstract}

Let $X$ be a simply connected complex manifold equipped with a real-analytic Kähler metric $g$. By the seminal work of Calabi's ([Ca], 1953), every germ of holomorphic isometry of $(X, g)$ into the projective space $\left(\mathbb{P}^{N}, d s_{\mathrm{FS}}^{2}\right), 1 \leq N \leq \infty$, equipped with the FubiniStudy metric extends to a holomorphic isometry on $(X, g)$.

In the current article we study the extension problem for germs of holomorphic isometries $f:\left(D ; x_{0}\right) \rightarrow\left(\Omega ; f\left(x_{0}\right)\right)$ up to normalizing constants between bounded domains in Euclidean spaces equipped with the Bergman metrics $d s_{D}^{2}$ on $D$ and $d s_{\Omega}^{2}$ on $\Omega$. Our basic extension results are of two types: extension results of the germ $\operatorname{Graph}(f) \subset D \times \Omega$ at $\left(x_{0}, f\left(x_{0}\right)\right)$ to a complex-analytic subvariety $S$ of $D \times \Omega$, and extension results on $S$ beyond the boundary of $D \times \Omega$ under certain assumptions. We call the former type interior extension results and the latter type boundary extension results. Interior extension follows from the work of Calabi [Ca] (cf. Remarks after the proof of Theorem 2.1.1). Our main focus will be on boundary extension for pairs of bounded domains $(D, \Omega)$ such that the Bergman kernel $K_{D}(z, w)$ extends meromorphically in $(z, \bar{w})$ to a neighborhood of $\bar{D} \times D$, and such that the analogous statement holds true for the Bergman kernel

N. Mok: The University of Hong Kong, Pokfulam Road, Hong Kong; e-mail: nmok@hku.hk

Mathematics Subject Classification (2010): 32D15, 32M15, 32Q15 
$K_{\Omega}(\zeta, \xi)$ on $\Omega$. Examples include pairs $(D, \Omega)$ of bounded symmetric domains in their Harish-Chandra realizations. The special case where $D$ is the unit disk $\Delta, \Omega$ is a polydisk $\Delta^{p}$, and $f:\left(\Delta, \lambda d s_{\Delta}^{2} ; 0\right) \rightarrow\left(\Omega, d s_{\Omega}^{2} ; 0\right)$ is a germ of holomorphic isometry in which the normalizing constant $\lambda$ is a positive integer $q$, was studied by Clozel-Ullmo ([CU], 2003 ) in connection to a problem in arithmetic dynamics. For such a germ of map they established a real-analytic functional identity arising from equating potential functions of Kähler metrics, and deduced as a consequence that the germ of subvariety $\operatorname{Graph}(f)$ at $(0,0)$ in $\Delta \times \Delta^{p}$ extends algebraically to $\mathbb{C} \times \mathbb{C}^{p}$. In their case the germ of holomorphic map $f$ arises from an algebraic correspondence on some finite-volume quotient of the unit disk, and, exploiting the action of the underlying lattice $\Gamma$ on an extension of $\operatorname{Graph}(f)$ to $\Delta \times \Delta^{p}$, they proved that $f$ must be totally geodesic, but conjectured ([CU, Conjecture 2.2 , p. 52]) that in fact any $f:\left(\Delta, q d s_{\Delta}^{2} ; 0\right) \rightarrow\left(\Delta^{p}, d s_{\Delta^{p}}^{2} ; 0\right)$ is totally geodesic.

To start with, we consider the case of $f:\left(D, \lambda d s_{D} ; 0\right) \rightarrow\left(\Omega, d s_{\Omega}^{2} ; 0\right)$ between bounded complete circular domains with base points at 0 . Generalizing the real-analytic functional identity expressed in terms of Bergman kernels, by polarization we obtain an infinite number of holomorphic identities, and the first question is to determine whether these identities are sufficiently non-degenerate to force analytic continuation. While examples show that in general this is not the case, we resolve the difficulty by studying deformations of simultaneous solutions of the holomorphic functional equations, and force analytic continuation by showing that, in the event that there are non-trivial deformations of simultaneous solutions to these equations, the germ of holomorphic isometry must take values in linear sections of the canonical image of the domain in the infinite-dimensional projective space $\mathbb{P}^{\infty}$, where the linear sections correspond to zeros of certain squareintegrable holomorphic functions which are in some sense extremal with respect to the Bergman metric. For a bounded complete circular domain $G \Subset \mathbb{C}^{m}$ with Bergman kernel $K_{G}(z, w)$, the domains of definition of $K_{D, w}:=K_{G}(z, w)$ grow to $\mathbb{C}^{n}$ as $w$ shrinks to 0 . Using this we prove the analytic continuation of $\operatorname{Graph}(f) \subset D \times \Omega$ to a complexanalytic subvariety $S^{\sharp}$ in the Euclidean space. In the special case of bounded symmetric domains in their Harish-Chandra realizations, we prove the following stronger result.

Theorem 1.3.1. Let $D \Subset \mathbb{C}^{n}$ and $\Omega \Subset \mathbb{C}^{N}$ be bounded symmetric domains in their Harish-Chandra realizations. Let $\lambda$ be any positive real number and $f:\left(D, \lambda d s_{D}^{2} ; 0\right) \rightarrow$ $\left(\Omega, d s_{\Omega}^{2} ; 0\right)$ be a germ of holomorphic isometry at $0 \in D$. Then the germ $\operatorname{Graph}(f)$ at $(0,0)$ extends to an affine-algebraic subvariety $S^{\sharp} \subset \mathbb{C}^{n} \times \mathbb{C}^{N}$ such that $S:=S^{\sharp} \cap(D \times \Omega)$ is the graph of a proper holomorphic isometric embedding $F: D \rightarrow \Omega$ extending the germ of holomorphic map $f$.

Bounded symmetric domains provide a first source of holomorphic isometries up to normalizing constants. A holomorphic totally geodesic embedding $F: D \rightarrow \Omega$ between bounded symmetric domains is a holomorphic isometry with respect to the Bergman metric up to a rational normalizing constant whenever $D$ is irreducible. In terms of Borel embeddings, $F$ extends algebraically to a holomorphic map between the dual Hermitian symmetric manifolds of the compact type, thus to rational maps on Euclidean spaces when $D \Subset \mathbb{C}^{n}$ and $\Omega \Subset \mathbb{C}^{N}$ are bounded symmetric domains in their Harish-Chandra realizations. At the same time, holomorphic totally geodesic embeddings of bounded symmetric 
domains into homogeneous disk bundles over them give examples of holomorphic isometries with any prescribed normalizing isometric real constant $\lambda>1$. On the other hand we produce examples of holomorphic isometric embeddings of the Poincaré disk into certain bounded symmetric domains $\Omega$ which are not totally geodesic. More precisely, we prove (cf. (3.2) for the meaning of 'congruence')

Theorem 3.2.1. For every positive integer $p>1$ there exists a holomorphic isometric embedding $F:\left(\Delta, d s_{\Delta}^{2}\right) \rightarrow\left(\Delta^{p}, d s_{\Delta^{p}}^{2}\right), F=\left(F_{1}, \ldots, F_{p}\right)$, where each component $F_{k}$, $1 \leq k \leq p$, is non-constant, such that $F$ is not totally geodesic. In particular, Conjecture 2.2 of Clozel-Ullmo [CU] is false. Furthermore, for $p \geq 3$ there exists a realanalytic 1-parameter family of mutually incongruent holomorphic isometric embeddings $F_{t}:\left(\Delta, d s_{\Delta}^{2}\right) \rightarrow\left(\Delta^{p}, d s_{\Delta^{p}}^{2}\right), t \in \mathbb{R}$.

It is in general an interesting problem to construct non-standard holomorphic isometric embeddings of the Poincaré disk $\Delta$ into bounded domains $\Omega$, including the case where $\Omega$ is a bounded symmetric domain. For the special case where $\Omega$ is the polydisk $\Delta^{p}, p \geq 2$, the classification problem has been posed, but only very partial results are known $(\mathrm{Ng}$ $[\mathrm{Ng}]$ ), including a complete classification for $p=2,3$. As a further example we also give an explicit construction of a non-trivial (proper) holomorphic isometric embedding $F: \Delta \rightarrow \mathcal{H}_{3}$ of the Poincaré disk into the Siegel upper half-plane $\mathcal{H}_{3}$ of genus 3. We will show that the latter is distinguishable from a holomorphic isometry into a polydisk by checking that the branch points of $F$ do not lie on the Shilov boundary $\operatorname{Sh}\left(\mathcal{H}_{3}\right)$ and invoking results of $\mathrm{Ng}[\mathrm{Ng}]$. It is also interesting to find domains $D$ other than the Poincaré disk admitting non-standard holomorphic isometric embeddings into some bounded domain $\Omega$. Restricted to the case where both $D$ and $\Omega$ are assumed to be bounded symmetric domains, the main interest lies in $D=B^{n}, n \geq 2$. For a discussion of this and related problems cf. the survey article Mok [Mk5, §5].

Our study of extensions of germs of holomorphic isometries generalizes to those between arbitrary bounded domains. Interior extension holds true unconditionally, while boundary extension holds true under certain conditions on Bergman kernels, as given by

Theorem 2.1.2 (main part). Let $D \Subset \mathbb{C}^{n}$ and $\Omega \Subset \mathbb{C}^{N}$ be bounded domains. Let $x_{0} \in D$, $y_{0} \in \Omega, \lambda$ be a positive real number and $f:\left(D, \lambda d s_{D}^{2} ; x_{0}\right) \rightarrow\left(\Omega, d s_{\Omega}^{2} ; y_{0}\right)$ be a germ of holomorphic isometry. Suppose furthermore that the Bergman kernel $K_{D}(z, w)$ extends as a meromorphic function of $(z, \bar{w})$ to a neighborhood of $\bar{D} \times D$ and $K_{\Omega}(\zeta, \xi)$ extends as a meromorphic function of $(\zeta, \bar{\xi})$ to a neighborhood of $\bar{\Omega} \times \Omega$. Then there exists a neighborhood $D^{\sharp}$ of $\bar{D}$ and a neighborhood $\Omega^{\sharp}$ of $\bar{\Omega}$ such that the germ of $\operatorname{Graph}(f) \subset$ $D \times \Omega$ at $\left(x_{0}, y_{0}\right)$ extends to an irreducible complex-analytic subvariety $S^{\sharp}$ of $D^{\sharp} \times \Omega^{\sharp}$.

Theorem 2.1.2 further generalizes to relatively compact domains on complex manifolds provided that the domains admit Bergman metrics and the canonical maps on them are embeddings (cf. 2.2).

Holomorphic isometries between bounded domains are meaningful for the study of holomorphic functions on such domains. As an illustration a bona fide holomorphic isometric embedding $F:\left(D, d s_{D}^{2}\right) \rightarrow\left(\Omega, d s_{\Omega}^{2}\right)$ between bounded circular domains starshaped with respect to 0 , with $F(0)=0$, is induced by a Hilbert space isomorphism 
$\mu: H^{2}(D) \rightarrow H^{2}(\Omega)$ onto the orthogonal complement of the Hilbert subspace $E \subset$ $H^{2}(\Omega)$ consisting of the functions vanishing on $Z:=F(D)$, yielding for holomorphic functions square-integrable on $Z$ (with respect to the measure induced from $D$ ) normpreserving holomorphic extensions to $\Omega$ square-integrable with respect to the Lebesgue measure.

\section{Extension of germs of holomorphic isometries with respect to the Bergman metric on bounded complete circular domains}

\subsection{Extension of germs of holomorphic isometries via holomorphic functional equations}

In connection to a problem in arithmetic dynamics, Clozel-Ullmo [CU] considered a germ of holomorphic isometry $f:\left(\Delta, q d s_{\Delta}^{2} ; 0\right) \rightarrow\left(\Delta, d s_{\Delta}^{2} ; 0\right)^{p}$ from the unit disk $\Delta$ into a polydisk, where $q$ is a positive integer. (Here and in what follows, for a bounded domain $D, d s_{D}^{2}$ stands for the Bergman metric.) They obtained a real-analytic functional identity arising from Kähler potentials, and proceeded from there to prove that Graph $(f)$ extends to an affine-algebraic subvariety. In higher dimensions the method of [CU] is difficult to generalize directly. In Mok [Mk3] we considered the analogous problem for the complex unit ball $B^{n}$. There, by polarization we obtained instead a continuous family of holomorphic functional identities, and we solved the problem for $B^{n}$, $n \geq 2$, by forcing analytic continuation by means of these identities. Here we formulate the starting point of our argument more generally for germs of holomorphic isometries between bounded complete circular domains, allowing at the same time the normalizing constant $\lambda$ to be any positive real number. Recall that a circular domain $D \subset \mathbb{C}^{n}$ is a domain invariant under the action of the circle group $S^{1}$ given by $\Phi: S^{1} \times D \rightarrow D$, $\Phi\left(e^{i \theta}, z\right)=e^{i \theta} z, \theta \in \mathbb{R}$. The domain $D$ is complete if and only if $0 \in D$. For a bounded complete circular domain $D \Subset \mathbb{C}^{n}$ and for $\theta \in \mathbb{R}$, the Bergman kernel $K_{D}(\cdot, \cdot)$ satisfies $K_{D}\left(e^{i \theta} z, e^{i \theta} w\right)=K_{D}(z, w)$, so that $K_{D}(z, 0)=K_{D}\left(e^{i \theta} z, 0\right)$, implying that $K_{D}(z, 0)$ is a (positive) constant.

Proposition 1.1.1. Let $D \Subset \mathbb{C}^{n}$ and $\Omega \Subset \mathbb{C}^{N}$ be bounded complete circular domains. Denote by $d s_{D}^{2}$, resp. $d s_{\Omega}^{2}$, the Bergman metric on $D$, resp. $\Omega$, and by $K_{D}$, resp. $K_{\Omega}$, the Bergman kernel on $D$, resp. $\Omega$. Let $\lambda$ be any positive real number and $f:\left(D, \lambda d s_{D}^{2} ; 0\right) \rightarrow$ $\left(\Omega, d s_{\Omega}^{2} ; 0\right)$ be a germ of holomorphic isometry at $0 \in D$. Then there exists some real number $A>0$ such that for $z, w \in D$ sufficiently close to 0 we have

$$
\begin{aligned}
K_{\Omega}(f(z), f(z)) & =A \cdot K_{D}(z, z)^{\lambda}, \quad \text { and hence } \\
K_{\Omega}(f(z), f(w)) & =A \cdot K_{D}(z, w)^{\lambda}, \quad \text { where } \quad K_{D}(z, w)^{\lambda}=e^{\lambda \log K_{D}(z, w)},
\end{aligned}
$$

in which log denotes the principal branch of logarithm.

Proof. Following the argument of Clozel-Ullmo [CU], we have from the hypothesis

$$
\begin{aligned}
\sqrt{-1} \partial \bar{\partial} \log K_{\Omega}(f(z), f(z)) & =\lambda \sqrt{-1} \partial \bar{\partial} \log K_{D}(z, z), \\
\log K_{\Omega}(f(z), f(z)) & =\lambda \log K_{D}(z, z)+\operatorname{Re}(\psi)
\end{aligned}
$$


for some holomorphic function $\psi$. Consider the Taylor expansion of $\log K_{D}(z, z)$ in $z_{1}, \ldots, z_{n}$ and $\overline{z_{1}}, \ldots, \overline{z_{n}}$. For a multi-index $I=\left(i_{1}, \ldots, i_{n}\right)$ with $i_{1}, \ldots, i_{n} \geq 0$, we write $z^{I}=z_{1}^{i_{1}} \cdots z_{n}$ and $|I|=i_{1}+\cdots+i_{n}$. By the invariance of the Bergman kernel under the circle group action $\left(e^{i \theta}, z\right) \mapsto e^{i \theta} z, \theta \in \mathbb{R}$, the coefficient of $z^{I} \overline{z^{J}}$ is zero whenever $|I| \neq|J|$. The analogue is also true for the complete circular domain $\Omega$. Since $f(0)=0$, it follows by substitution that in the Taylor expansion of $\log K_{\Omega}(f(z), f(z))$ at 0 , the coefficients of terms of pure type $z^{I}$ and $\overline{z^{I}}$ must vanish for any $I=\left(i_{1}, \ldots, i_{n}\right)$, $i_{1}, \ldots, i_{n} \geq 0$, such that at least one of the indices $i_{k}, 1 \leq k \leq n$, is non-zero. On the other hand, the Taylor expansion of $2 \operatorname{Re}(\psi)=\psi+\bar{\psi}$ at 0 consists precisely of terms of pure type, and it follows by comparing the two sides of (1) that $\operatorname{Re}(\psi)$ must be a (real) constant.

We now introduce holomorphic functional identities by polarization, viz.,

$$
\log K_{\Omega}(f(z), f(w))=\lambda \log K_{D}(z, w)+a+H(z, w),
$$

where $a$ is a real constant and

$$
H(z, w)=\sum_{(I, J) \neq(0,0)} H_{I \bar{J}} z^{I} \overline{w^{J}}
$$

is holomorphic in $z$ and anti-holomorphic in $w$. Recall that $K_{D}(0,0)$ and $K_{\Omega}(0,0)$ are real and 'log' stands for the principal branch of the logarithm. Restricting to the diagonal $\{z=w\}$ we have $H(z, z)=0$, i.e., $\sum_{(I, J) \neq(0,0)} H_{I \bar{J}} z^{I} \overline{z^{J}}=0$, so that $H_{I \bar{J}}=0$ for all $(I, J) \neq(0,0)$, hence $H(z, w)=0$ where defined, yielding

$$
\begin{aligned}
\log K_{\Omega}(f(z), f(w)) & =\lambda \log K_{D}(z, w)+a, \text { hence } \\
K_{\Omega}(f(z), f(w)) & =A \cdot K_{D}(z, w)^{\lambda},
\end{aligned}
$$

where $A:=e^{a}$ and $K_{D}(z, w)^{\lambda}=e^{\lambda \log K_{D}(z, w)}$, as desired.

For the application of Proposition 1.1.1 to extension problems, we recall first of all the following well-known fact about the Bergman kernel on a complete circular domain.

Lemma 1.1.1. Let $D \Subset \mathbb{C}^{n}$ be a complete circular domain and denote by $K_{D}(z, w)$ the Bergman kernel on D. Suppose $r$ is a real number, $0<r<1$, so that $r D \subset D$. Then for $z \in D$ and $w \in r D$ we have $K_{D}(z, w)=K_{D}(r z, w / r)$. In particular, for every $w \in r D$ the holomorphic function $K_{D, w}(z):=K_{D}(z, w)=K_{D}(r z, w / r):=K_{D, w / r}(r z)$ of $z \in D$ extends holomorphically to $(1 / r) D$ when we define $K_{D, w}(z):=K_{D, w / r}(r z)$ for $z \in(1 / r) D$.

Proof. From the invariance of $D$ under the circle group action $\left(e^{i \theta}, z\right) \mapsto e^{i \theta} z$ we have

$$
K_{D}(z, w)=\sum_{|I|=|J|} a_{I \bar{J}} z^{I} \overline{w^{J}}
$$

Observing that $(r z)^{I} \overline{(w / r)^{J}}=z^{I} \overline{w^{J}}$ whenever $|I|=|J|$, we have $K_{D}(z, w)=$ $K_{D}(r z, w / r)$ for $z \in D$ and $w \in r D$. Fixing $w_{0} \in r D, K_{D}\left(r z, w_{0} / r\right)$ is defined for 
$z \in(1 / r) D$. Hence, $K_{D, w_{0}}(z)=K_{D}\left(r z, w_{0} / r\right)$ extends holomorphically from $D$ to $(1 / r) D$, as desired.

For $r>0$ we write $D_{r}:=B^{n}(0 ; r)$. Choose $e>0$ such that $f:\left(D, \lambda d s_{D}^{2} ; 0\right) \rightarrow$ $\left(\Omega, d s_{\Omega}^{2} ; 0\right)$ is represented by a holomorphic embedding defined on $D_{e}$, and such that moreover $K_{D}(z, w)$ and $K_{\Omega}(f(z), f(w))$ are non-zero whenever $z, w \in D_{e}$. For notational convenience later on, we will also require that $e<1$. Similarly for $\rho>0$ we write $\Omega_{\rho}:=B^{N}(0 ; \rho)$. Choose $\delta_{0}$ such that $0<\delta_{0}<1$ and $\Omega_{\delta_{0}} \Subset \Omega$.

In Mok [Mk3] we studied germs of holomorphic isometries $f$ from the unit ball $B^{n}, n \geq 2$, to its Cartesian products in the case where the normalizing constant is a positive integer $q$. There, making use of the explicit form of the holomorphic functional identities arising from equating potential functions, we extended $\operatorname{Graph}(f)$ to an affinealgebraic subvariety. To prove an analogue for the general case we encounter first of all the problem that the associated functional identities are in general not sufficiently 'nondegenerate' to force analytic continuation. We overcome difficulties arising from such degenerate situations by imposing additional constraints to cut down the set of simultaneous solutions to the functional equations. Recall that $K_{D}(z, 0)=C>0$. Let $D^{\sharp} \Subset \mathbb{C}^{n}$ be a neighborhood of $\bar{D}$. By Lemma 1.1.1, there exists $\epsilon_{0}$ satisfying $0<\epsilon_{0}<e$ such that for any $w \in D_{\epsilon_{0}}=B^{n}\left(0 ; \epsilon_{0}\right), K_{D}(z, w)$ is defined for $z \in D^{\sharp}$ (by analytic extension of $\left.K_{D, w}(\cdot)=K_{D}(\cdot, w)\right)$, and $\operatorname{Re}\left(K_{D}(z, w)\right)>0$ for any $(z, w) \in D^{\sharp} \times D_{\epsilon_{0}}$. Then $K_{D}(z, w)^{\lambda}=e^{\lambda \log K_{D}(z, w)}$ is defined for $(z, w) \in D^{\sharp} \times D_{\epsilon_{0}}$. We will further assume that $f\left(D_{\epsilon_{0}}\right) \Subset \Omega_{\delta_{0}}$. Suppose now $0<\epsilon \leq \epsilon_{0}$. Instead of a germ of map, the symbol $f$ will sometimes stand for the map $f:\left(D_{\epsilon},\left.\lambda d s_{D}^{2}\right|_{D_{\epsilon}}\right) \rightarrow\left(\Omega, d s_{\Omega}^{2}\right)$. Thus, $K_{D}(z, w)^{\lambda}$ is defined on $D^{\sharp} \times D_{\epsilon}$ as a function holomorphic in $(z, \bar{w})$. Writing $K_{\Omega}(0,0)=: C^{\prime}$ and $A:=C^{\prime} C^{-\lambda}$, we have

Proposition 1.1.2. For each $w \in D_{\epsilon}$, let $V_{w} \subset D \times \Omega$ be the set of all $(z, \zeta) \in D \times \Omega$ such that

$$
\left(\mathbf{I}_{w}\right) \quad K_{\Omega}(\zeta, f(w))=A \cdot K_{D}(z, w)^{\lambda} .
$$

Define $V_{\epsilon}=\bigcap_{w \in D_{\epsilon}} V_{w}$. Suppose $\operatorname{dim}_{(z, f(z))}\left(V_{\epsilon} \cap(\{z\} \times \Omega)\right) \geq 1$ for a general point $z \in D_{\epsilon}$. Then there exists a family of holomorphic functions $h_{\alpha} \in H^{2}(\Omega), \alpha \in \mathbf{A}$, such that

$$
\operatorname{Graph}(f) \subset D_{\epsilon} \times E, \quad \text { where } E:=\bigcap_{\alpha \in \mathbf{A}} \operatorname{Zero}\left(h_{\alpha}\right),
$$

and $\operatorname{dim}_{(z, f(z))}\left(V_{\epsilon} \cap(\{z\} \times E)\right)=0$ for a general point $z \in D_{\epsilon}$.

By a general point on a complex manifold we mean the complement of a nowhere dense complex-analytic subvariety. By the Identity Theorem for holomorphic functions, $V_{\epsilon} \subset$ $D \times \Omega$ is independent of $\epsilon>0$, and we will write $V$ in place of $V_{\epsilon}$. We say that the system of functional equations $\left(\mathbf{I}_{w}\right), w \in D_{\epsilon}$, is sufficiently non-degenerate whenever any irreducible branch of $V$ containing $\operatorname{Graph}(f)$ must be of dimension $n=\operatorname{dim}(\operatorname{Graph}(f))$.

Proof of Proposition 1.1.2. Graph $(f) \subset D_{\epsilon} \times \Omega$ is by definition contained in $V$. By hypothesis, $\operatorname{dim}_{(z, f(z))}(V \cap(\{z\} \times \Omega))=: q \geq 1$ at a general point $z \in D_{\epsilon}$ (hence actually at any point $z \in D_{\epsilon}$ by upper semicontinuity of the fiber dimension). Fix a 
Stein neighborhood $\Omega_{0}$ of 0 in $\Omega$ such that $f\left(D_{\epsilon_{0}}\right) \subset \Omega_{0}$. (We may take for instance $\Omega_{0}=B^{N}\left(0 ; \delta_{0}\right)$.) Let $Z_{\epsilon} \subset V \cap\left(D_{\epsilon} \times \Omega_{0}\right)$ be an irreducible complex-analytic subvariety of $D_{\epsilon} \times \Omega_{0}$ containing $\operatorname{Graph}(f)$ such that $\operatorname{dim}_{(z, f(z))}\left(Z_{\epsilon} \cap\left(\{z\} \times \Omega_{0}\right)\right)=1$ for a general point $z \in D_{\epsilon}$. The subvariety $Z_{\epsilon} \subset V$ may be obtained by an inductive procedure, as follows. If $q=1$, it suffices to take $Z_{\epsilon}$ to be an irreducible component of $V \cap\left(D_{\epsilon} \times \Omega_{0}\right)$ containing $\operatorname{Graph}(f)$. If $q>1$, choose any $x_{1} \in V \cap\left(D_{\epsilon} \times \Omega_{0}\right)$ lying outside the subvariety $\operatorname{Graph}(f) \subset V \cap\left(D_{\epsilon} \times \Omega_{0}\right)$. Since $V \cap\left(D_{\epsilon} \times \Omega_{0}\right)$ is Stein, there exists a holomorphic function $g_{1}$ on $V \cap\left(D_{\epsilon} \times \Omega_{0}\right)$ such that $\left.g_{1}\right|_{\operatorname{Graph}(f)} \equiv 0$ and $g_{1}\left(x_{1}\right) \neq 0$. We now define $Z_{\epsilon}^{(n+q-1)} \subset V \cap\left(D_{\epsilon} \times \Omega_{0}\right)$ to be an irreducible component of the zero set $\operatorname{Zero}\left(g_{1}\right) \subset V \cap\left(D_{\epsilon} \times \Omega_{0}\right)$ of $g_{1}$ containing $\operatorname{Graph}(f)$. If $q=2$ we take $Z_{\epsilon}:=Z_{\epsilon}^{(n+1)}$. If $q>2$ we proceed further with $V \cap\left(D_{\epsilon} \times \Omega_{0}\right)$ replaced by $Z_{\epsilon}^{(n+q-1)}, x_{1}$ replaced by $x_{2} \epsilon$ $Z_{\epsilon}^{(n+q-1)}-\operatorname{Graph}(f)$ to find $g_{2}$ holomorphic on $Z_{\epsilon}^{(n+q-1)}$ such that $\left.g_{2}\right|_{\operatorname{Graph}(f)} \equiv 0$ and $g_{2}\left(x_{2}\right) \neq 0$. Proceeding inductively we reach $Z_{\epsilon}:=Z_{\epsilon}^{(n+1)}$ such that $Z_{\epsilon} \subset D_{\epsilon} \times \Omega$ is an irreducible subvariety containing $\operatorname{Graph}(f)$ and such that $\operatorname{dim}_{(z, f(z))}\left(Z_{\epsilon} \cap(\{z\} \times \Omega)\right)=1$ at a general point $z \in D_{\epsilon}$.

Write $v: \widetilde{Z}_{\epsilon} \rightarrow Z_{\epsilon}$ for the normalization of $Z_{\epsilon}$. Since the singular set of $\widetilde{Z}_{\epsilon}$ is of codimension $\geq 2$, and $v^{-1}(\operatorname{Graph}(f)) \subset \widetilde{Z}_{\epsilon}$ is of pure codimension 1 , a general point $\tilde{p}$ of any irreducible branch $B$ of $v^{-1}(\operatorname{Graph}(f))$ is a smooth point of $\widetilde{Z}_{\epsilon}$. We may choose $\widetilde{p}$ to be also a smooth point of $B$ such that $\left.\nu\right|_{B}: B \rightarrow \operatorname{Graph}(f)$ is a local biholomorphism at $\tilde{p}$. Write $p:=v(\tilde{p}), p=\left(z_{0}, f\left(z_{0}\right)\right) \in D_{\epsilon} \times \Omega$, and denote by $\pi_{D}: D \times \Omega \rightarrow D$ the canonical projection. Choose a neighborhood $W$ of $\widetilde{p}$ in $\widetilde{Z}_{\epsilon}$ and a neighborhood $U$ of $z_{0}$ in $D_{\epsilon}$ such that $\left.\pi_{D} \circ \nu\right|_{W \cap B}: W \cap B \rightarrow U$ is a biholomorphism which extends to a biholomorphism $\sigma: W \rightarrow U \times \Delta$ when $U$ is identified with $U \times\{0\}$. (A neighborhood is always understood to be connected.) Write $v\left(\sigma^{-1}(z, t)\right)=(h(z, t), g(z, t))$. Since $h$ is a holomorphic submersion at $\left(z_{0}, 0\right)$, it remains a holomorphic submersion at $(z, t)$ sufficiently close to $\left(z_{0}, 0\right)$, and without loss of generality we may choose $W, U$ and $v$ such that $h(z, t)=z$. For $t \in \Delta$, write $f_{t}(z)=g(z, t)$. We have

$$
K_{\Omega}\left(f_{t}(z), f(w)\right)=A \cdot K_{D}(z, w)^{\lambda}
$$

such that $f_{0}(z)=f(z)$. Assume that $\left.\frac{\partial^{k}}{\partial t^{k}} f_{t}(z)\right|_{t=0} \equiv 0$ for $k<\ell$ and $\eta(f(z)):=$ $\left.\frac{\partial^{\ell}}{\partial t^{\ell}} f_{t}(z)\right|_{t=0} \not \equiv 0$. Let $\left(h_{j}\right)_{j=0}^{\infty}$ be an orthonormal basis of $H^{2}(\Omega)$. We have

$$
K_{\Omega}\left(f_{t}(z), f(w)\right)=\sum_{j} h_{j}\left(f_{t}(z)\right) \overline{h_{j}(f(w))}=A \cdot K_{D}(z, w)^{\lambda}
$$

for every $t$. Hence, differentiating both sides of (1) $\ell$ times against $t$ and noting that the right-hand side is independent of $t$, we have

$$
\begin{gathered}
\left.\frac{\partial^{\ell}}{\partial t^{\ell}} K_{\Omega}\left(f_{t}(z), f(w)\right)\right|_{t=0} \equiv 0, \quad \text { i.e., } \\
\sum_{i, j} \frac{\partial h_{j}}{\partial \zeta_{i}} \frac{\partial^{\ell} f_{t}^{i}}{\partial t^{\ell}}(z) \overline{h_{j}(f(w))} \equiv 0, \quad \text { i.e., } \quad \sum_{j} d h_{j}(\eta(f(z))) \overline{h_{j}(f(w))} \equiv 0 .
\end{gathered}
$$


Denote by $\mathbb{H}$ the separable Hilbert space of square-integrable sequences of complex numbers. Let $\Phi: \Omega \rightarrow \mathbb{H}$ be defined by

$$
\Phi(\zeta)=\left(h_{0}(\zeta), h_{1}(\zeta), \ldots\right)
$$

By the choice of $\epsilon, f$ is injective on $D_{\epsilon}$, hence $\left.f\right|_{U}: U \rightarrow \Omega$ is a holomorphic embedding onto a locally closed complex submanifold $\Sigma$. In terms of the Hermitian inner product $\left\langle\cdot,{ }^{-}\right\rangle$on the Hilbert space $\mathbb{H}$, the identity (2) is given by

$$
\langle d \Phi(\eta(f(z))), \overline{\Phi(f(w))}\rangle=0,
$$

where $\eta(f(z))$ is interpreted as a vector field along $\Sigma$ and $d \Phi(\eta)$ as a vector field along $\Xi:=\Phi(\Sigma) \subset \mathbb{H}$. In other words, we have a non-trivial holomorphic vector field along $\Xi$ which is orthogonal to the linear span of $\Phi(f(w))$ as $w$ ranges over $D_{\epsilon}$. We may assume $\eta\left(f\left(z_{0}\right)\right) \neq 0$. Let $h_{0}$ be chosen so that $\left|h\left(f\left(z_{0}\right)\right)\right|$ attains its maximum value at $h=h_{0}$ among all $h \in H^{2}(\Omega)$ of unit norm. Choose $h_{1} \perp h_{0}$ such that $\left|d h\left(\eta\left(f\left(z_{0}\right)\right)\right)\right|$ attains its maximum value at $h=h_{1}$ among all $h \in H^{2}(\Omega)$ of unit norm and orthogonal to $h_{0}$. Then, for any $h$ such that $h \perp h_{0}$ and $h \perp h_{1}$ we have $h\left(f\left(z_{0}\right)\right)=0$ and $d h\left(\eta\left(f\left(z_{0}\right)\right)\right)=0$. Thus, completing $\left(h_{0}, h_{1}\right)$ to any orthonormal basis $\left(h_{0}, h_{1}, \ldots\right)$ of $H^{2}(\Omega)$, for all $w$ in $D_{\epsilon}$ we derive from (2) that

$$
d h_{0}\left(\eta\left(f\left(z_{0}\right)\right)\right) \overline{h_{0}(f(w))}+d h_{1}\left(\eta\left(f\left(z_{0}\right)\right)\right) \overline{h_{1}(f(w))}=0 .
$$

Substituting $w=z_{0}, h_{1}\left(f\left(z_{0}\right)\right)=0$ and $h_{0}\left(f\left(z_{0}\right)\right) \neq 0$ imply that $d h_{0}\left(\eta\left(f\left(z_{0}\right)\right)\right)=0$. Since $\mid d h\left(\left(\eta\left(f\left(z_{0}\right)\right)\right) \mid\right.$ attains its maximum among $h \perp h_{0}$ of unit norm at $h=h_{1}$, we must have $d h_{1}\left(\eta\left(f\left(z_{0}\right)\right)\right) \neq 0$, and it follows from (3) that $h_{1}(f(w))=0$. Writing $\left(x_{0}, x_{1}, \ldots\right)$ for a point in $\mathbb{H}$ we conclude that $\Phi(f(U))$ lies in a hyperplane section which is the zero set of a continuous linear functional on $\mathbb{H}$, given by

$$
\Phi(f(U)) \subset\left\{x_{1}=0\right\} \subset \mathbb{H} .
$$

Note that the function $h_{\alpha}=h_{1, z_{0}}$ is defined on all of $\Omega$. Consider all deformations $f_{t}(z)=$ $g(t, z)$ on some domain $U \subset D_{\epsilon}$ defined as above, and denote by $\mathbf{A}$ the set of indices $\alpha$ for all functions $h_{\alpha}$ thus obtained. Define $\mathcal{E}:=\left\{h_{\alpha} \in H^{2}(\Omega): \alpha \in \mathbf{A}\right\}$ and denote by $E \subset \Omega$ the common zero set of all $h_{\alpha} \in \mathcal{E}$. Thus $\Phi(E) \subset \mathbb{H}$ is a (closed) linear section of $\Phi(\Omega)$ containing $\Xi$. Now consider the functional equations $\left(\mathbf{I}_{w}\right), w \in D_{\epsilon}$, together with a restriction on the indeterminate $\zeta$, given by

$$
K_{\Omega}(\zeta, f(w))=A \cdot K_{D}(z, w)^{\lambda}, \quad \zeta \in E .
$$

For the proof of Proposition 1.1.2 it remains to prove that

$$
\left(\dagger \operatorname{dim}_{(z, f(z))}(V \cap(\{z\} \times E))=0\right.
$$

for a general point $z \in D_{\epsilon}$. Suppose otherwise. Repeating the same argument as above, we obtain a holomorphic 1-parameter family $\left\{f_{t}\right\}_{t \in \Delta}, f_{0}=f$, defined on some domain $U \subset D_{\epsilon}$ such that $f_{t}$ takes values in $E$, thereby deriving the existence of a holomorphic vector field $\eta$ along $\Sigma=f(U)$ and $h_{1} \in \mathcal{E}$ such that $d h_{1}\left(\eta\left(f\left(z_{0}\right)\right)\right) \neq 0$ for a general 
point $z_{0} \in U$. By definition $h_{1}$ must vanish identically on $E$, hence $h_{1}\left(f_{t}(z)\right)=0$ for $z \in U$ and for $t \in \Delta$. Now, differentiating the latter identity $\ell$ times against $t$ we conclude that $d h_{1}\left(\eta\left(f\left(z_{0}\right)\right)\right)=0$, contradicting the choice of $h_{1}$. Thus, we have established ( $\dagger$ ) by contradiction, proving Proposition 1.1.2.

Example (functional equations are not sufficiently non-degenerate). The following example shows that the situation where the system of holomorphic functional equations are not sufficiently 'non-degenerate' does occur. In other words, the example is one for which $\operatorname{dim}_{(z, f(z))}(V \cap(\{z\} \times \Omega)) \geq 1$. Let $N>n \geq 1$ be integers and consider the totally geodesic holomorphic isometric embedding $f:\left(B^{n}, \frac{N+1}{n+1} d s_{B^{n}}^{2}\right) \rightarrow\left(B^{N}, d s_{B^{N}}^{2}\right)$ given by $f(z)=(z, 0)$ for $z=\left(z_{1}, \ldots, z_{n}\right)$. In this case the holomorphic functional equations relating Bergman kernels are given by

$$
K_{B^{N}}(\zeta, f(w))=A \cdot K_{B^{n}}(z, w)^{\frac{N+1}{n+1}}
$$

for some $A>0$. Denoting by $\langle\cdot, \bar{\cdot}\rangle$ the Euclidean Hermitian inner product, we have $K_{B^{m}}(z, w)=c_{m}(1-\langle z, \bar{w}\rangle)^{-(m+1)}$ for some constant $c_{m}>0$. We thus have

$$
\frac{c_{N}}{(1-\langle\zeta, \overline{(w, 0)}\rangle)^{N+1}}=A\left(\frac{c_{n}}{(1-\langle z, \bar{w}\rangle)^{n+1}}\right)^{\frac{N+1}{n+1}}
$$

Substituting $(z, w)=(0,0)$ and $\zeta=f(0)=0$ we have $c_{N}=A c_{n}^{(N+1) /(n+1)}$. For $w$ sufficiently small and $z \in B^{n}$, the functional equation (1) on $\zeta$ is equivalent to

$$
1-\langle\zeta, \overline{(w, 0)}\rangle=1-\langle z, \bar{w}\rangle
$$

for $\zeta$ sufficiently close to $(z, 0)$. Clearly $\zeta=f(z)=(z, 0)$, which describes the image of the holomorphic isometry, satisfies the functional equations (2). However, when $(z, w)$ is fixed and we put $\zeta=\left(z, z^{\prime}\right)$, where $z^{\prime} \in \mathbb{C}^{N-n}$ is arbitrary, (2) remains satisfied. In fact, these $\zeta$ give all possible simultaneous solutions to (2), and we have

$$
V=\left\{(z, \zeta) \in B^{n} \times B^{N}: \zeta=\left(z, z^{\prime}\right), z^{\prime} \in \mathbb{C}^{N-n}\right\},
$$

hence $\operatorname{dim}_{(z, f(z))}\left(V \cap\left(\{z\} \times B^{N}\right)\right)=N-n \geq 1$. Infinitesimal variations $\eta$ of simultaneous solutions $f_{t}(z)=\left(z, g_{t}(z)\right), g_{0}(z) \equiv 0$, to (3) are of the form

$$
\eta(f(z))=\eta(z, 0)=\sum_{\ell=n+1}^{N} a_{\ell}(z) \frac{\partial}{\partial \zeta_{\ell}},
$$

where $a_{\ell}(z)$ are holomorphic functions in $z$ defined on some non-empty open subset $U \subset B^{n}$. Here the fiber of the canonical projection $\pi: V \rightarrow B^{n}$ over a general point $z \in B^{n}$ can be cut down to an isolated point when we impose the conditions $\zeta_{n+1}=\cdots=\zeta_{N}=0$, which in fact corresponds to cutting $B^{N}$ by zero sets of extremal functions maximizing the derivatives in the direction $\partial / \partial \zeta_{\ell}, n+1 \leq \ell \leq N$, at $(z, 0) \in B^{N}$. 
In the proof of Proposition 1.1.2, in the case where $\left(\mathbf{I}_{w}\right), w \in D_{\epsilon}$, are not sufficiently non-degenerate, we have to consider extremal functions $h \in \mathcal{E}$. Since these functions will play a crucial role in extension problems in the rest of the article, we will now prove a number of their basic properties. Recall the initial choice of $\epsilon_{0}>0$ as specified in the paragraph preceding Proposition 1.1.2. The set $\mathcal{E} \subset H^{2}(\Omega)$ of extremal functions depends on the choice of $\epsilon>0,0<\epsilon \leq \epsilon_{0}$. We will write $\mathcal{E}(\epsilon), \mathbf{A}(\epsilon), E(\epsilon)$ to indicate this dependence, and from now on write $\mathcal{E}=\mathcal{E}\left(\epsilon_{0}\right), E=E\left(\epsilon_{0}\right)$ and regard $f$ as being defined on $D_{\epsilon_{0}}$.

Note that each $h \in \mathcal{E}(\epsilon)$ is of the form $h_{1}$ in the notation of the proof of Proposition 1.1.2. More precisely, given a holomorphic 1-parameter family $\left\{f_{t}\right\}_{t \in \Delta}$ defined on a domain $U \subset D_{\epsilon}$ obtained as a deformation of $f_{0}=\left.f\right|_{U}$ of simultaneous solutions of the holomorphic functional equations $\left(\mathbf{I}_{w}\right)$, by differentiation we obtain a holomorphic vector field $\eta$ defined along $\Sigma=f(U) \subset \Omega$, and, for each $z_{0} \in U$ we have an $h_{1}$ which is determined by $\eta$ and by the choice of $z_{0}$. We write $h_{1}=h_{\eta, z_{0}}$. We are going to relate $h_{1}$ to the Bergman kernel $K_{\Omega}$ on $\Omega$, thereby extending its domain of definition by means of properties of Bergman kernels on complete circular domains as given in Lemma 1.1.1.

Recall that $h_{0} \in H^{2}(\Omega)$ has been chosen such that, among all $h \in H^{2}(\Omega)$ of unit norm, the maximum of $\left|h\left(f\left(z_{0}\right)\right)\right|$ is attained at $h=h_{0}$. Moreover, $h_{1} \in H^{2}(\Omega)$ has been chosen such that, among all $h \in H^{2}(\Omega)$ of unit norm and orthogonal to $h_{0}$, the maximum of $\left|d h\left(\eta\left(f\left(z_{0}\right)\right)\right)\right|$ is attained at $h=h_{1}$. Both $h_{0}$ and $h_{1}=h_{\eta, z_{0}}$ are uniquely determined only up to a scalar constant of modulus 1 . We have

Lemma 1.1.2. The extremal function $h_{1}=h_{\eta, z_{0}} \in \mathcal{E}$ can be expressed in terms of the Bergman kernel $K_{\Omega}$ as

$$
h_{1}(\zeta)=\frac{\overline{\partial_{\eta\left(f\left(z_{0}\right)\right)} K_{\Omega}\left(f\left(z_{0}\right), \zeta\right)}-\left(\overline{\partial_{\eta\left(f\left(z_{0}\right)\right)} h_{0}}\right) h_{0}(\zeta)}{\overline{\partial_{\eta\left(f\left(z_{0}\right)\right)} h_{1}}} .
$$

Furthermore, if we choose the unique $h_{1}=h_{\eta, z_{0}}$ such that $d h_{1}(\eta) \neq 0$ is (real and) positive, then, with the vector field $\eta$ along $\Sigma \subset \Omega$ being fixed and $h_{1}=h_{\eta, z}$ depending on the base point $z \in U \subset D_{\epsilon_{0}}, h_{\eta, z}(\zeta)$ varies real-analytically in $(z, \zeta)$.

Here in $\partial_{\eta\left(f\left(z_{0}\right)\right)} K_{\Omega}\left(f\left(z_{0}\right), \xi\right)$, the notation $\eta\left(f\left(z_{0}\right)\right)$ signifies the (1,0)-tangent vector $\left(\eta\left(f\left(z_{0}\right)\right), 0\right)$ at $\left(f\left(z_{0}\right), \xi\right) \in \Omega \times \Omega$, and $\partial_{\eta\left(f\left(z_{0}\right)\right)} h_{0}$ means $\partial_{\eta\left(f\left(z_{0}\right)\right)} h_{0}\left(f\left(z_{0}\right)\right)$, etc. We will call $h_{1}=h_{\eta, z_{0}}$ a normalized extremal function to mean that $d h_{1}(\eta)$ is positive.

Proof of Lemma 1.1.2. Complete $\left(h_{0}, h_{1}\right)$ to an orthonormal basis $\left(h_{0}, h_{1}, \ldots\right)$ of $H^{2}(\Omega)$. From the expansion of $K_{\Omega}$ in terms of the chosen orthonormal basis, for $\zeta, \xi \in \Omega$,

$$
K_{\Omega}(\zeta, \xi)=h_{0}(\zeta) \overline{h_{0}(\xi)}+h_{1}(\zeta) \overline{h_{1}(\xi)}+\cdots
$$

Note that $K_{\Omega}(\xi, \zeta)=\overline{K_{\Omega}(\zeta, \xi)}$. Substituting $\zeta=f\left(z_{0}\right)$ in (1) and using the fact that $h_{j}\left(f\left(z_{0}\right)\right)=0$ whenever $j \geq 1$, we deduce

$$
K_{\Omega}\left(f\left(z_{0}\right), \xi\right)=h_{0}\left(f\left(z_{0}\right)\right) \overline{h_{0}(\xi)},
$$


so that

$$
h_{0}(\xi)=\frac{K_{\Omega}\left(\xi, f\left(z_{0}\right)\right)}{\overline{h_{0}\left(f\left(z_{0}\right)\right)}},
$$

expressing $h_{0}$ in terms of $f$ and $K_{\Omega}$. Furthermore, differentiating both sides of (1) against $\eta\left(f\left(z_{0}\right)\right)$ and using the fact that $d h_{j}\left(\left(\eta\left(f\left(z_{0}\right)\right)\right)=0\right.$ whenever $j \geq 2$ we have

$$
\partial_{\eta\left(f\left(z_{0}\right)\right)} K_{\Omega}\left(f\left(z_{0}\right), \xi\right)=\left(\partial_{\eta\left(f\left(z_{0}\right)\right)} h_{0}\right) \overline{h_{0}(\xi)}+\left(\partial_{\eta\left(f\left(z_{0}\right)\right)} h_{1}\right) \overline{h_{1}(\xi)}
$$

so that

$$
h_{1}(\zeta)=\frac{\overline{\partial_{\eta\left(f\left(z_{0}\right)\right) K_{\Omega}\left(f\left(z_{0}\right), \zeta\right)}}-\left(\overline{\partial_{\eta\left(f\left(z_{0}\right)\right)} h_{0}}\right) h_{0}(\zeta)}{\overline{\partial_{\eta\left(f\left(z_{0}\right)\right)} h_{1}}},
$$

where we replace $\xi$ in (3) by $\zeta$ in (4), proving the first half of Lemma 1.1.2.

For the proof of the last statement of Lemma 1.1.2, we may also fix the choice of $h_{0}$ by requiring $h_{0}(z)$ to be (real and) positive. By the formulas (2) and (4) it suffices to check that $h_{0}(f(z))$ (with a hidden dependence of $h_{0}$ on $z$ ) and $d h_{1}(\eta)=\partial_{\eta(f(z))} h_{1}$ both depend real-analytically on $z$. Now from $K_{\Omega}(f(z), f(z))=\left|h_{0}(f(z))\right|^{2}$ (by (2)) and the normalization that $h_{0}(f(z))$ is positive it follows that $h_{0}(f(z))=\sqrt{K_{\Omega}(f(z), f(z))}$ depends real-analytically on $z$. On the other hand from (1) by differentiation against $\eta$ in the $\zeta$ variable and then against $\bar{\eta}$ in the $\xi$ variable and evaluating at $(f(z), f(z))$ it follows that $\left|\partial_{\eta(f(z))} h_{1}\right|^{2}$ can be expressed as a real-analytic function of $z$, noting that $h_{j}(f(z))=d h_{j}(f(z))=0$ whenever $j \geq 2$ so that $h_{2}, h_{3}, \ldots$ do not enter into the formula for $\left|\partial_{\eta(f(z))} h_{1}\right|^{2}$, and $\partial_{\eta(f(z))} h_{1}$ varies real-analytically in $z$ by our normalization that $\partial_{\eta(f(z))} h_{1}$ is real and positive, proving Lemma 1.1.2.

For the tangent bundle $\pi: T_{\Omega} \rightarrow \Omega$ we denote by $T_{\Omega}^{\prime} \subset T_{\Omega}$ the subset of non-zero tangent vectors. In general, for $\tau \in T_{\Omega}^{\prime}$ we have the notion of an extremal function adapted to $\tau$, meaning an element $h_{\tau} \in H^{2}(\Omega)$ of unit norm such that $d h(\tau)$ attains maximal modulus at $h=h_{\tau}$ among all $h \in H^{2}(\Omega)$ of unit norm satisfying $h(\pi(\tau))=0$. Such an $h_{\tau}$ is unique up to multiplication by a scalar of unit modulus. As above, we can fix $h_{\tau}$ by requiring that $d h_{\tau}(\tau)$ is real and positive, and we call $h_{\tau} \in H^{2}(\Omega)$ the normalized extremal function adapted to $\tau \in T_{\Omega}^{\prime}$. For a real-analytic manifold $X$, we will say that a mapping $\mathfrak{B}: X \rightarrow H^{2}(\Omega)$ is separately real-analytic to mean that $\mathfrak{B}(x)\left(\zeta_{0}\right)$ is realanalytic in $x \in X$ for any $\zeta_{0} \in \Omega$. Obviously the Identity Theorem holds true for $\mathfrak{B}$ in the sense that $\mathfrak{B} \equiv 0$ whenever $\mathfrak{B}$ vanishes on a non-empty open subset $U \subset X$. Denote by $\mathfrak{H}: T_{\Omega}^{\prime} \rightarrow H^{2}(\Omega)$ the mapping defined by $\mathfrak{H}(\tau)=h_{\tau}$ and denote its image by $\mathfrak{X}(\Omega) \subset H^{2}(\Omega)$. From the formula for $h_{\tau}$ implicit in Lemma 1.1.2, the mapping $\mathfrak{h}: \Omega \times T_{\Omega}^{\prime} \rightarrow \mathbb{C}$ defined by $\mathfrak{h}(\zeta, \tau)=h_{\tau}(\zeta)$ is holomorphic in $\zeta$ and real-analytic in $(\zeta, \tau)$, thus $\mathfrak{H}: T_{\Omega}^{\prime} \rightarrow H^{2}(\Omega)$ is separately real-analytic.

For the further study of extremal functions $h_{\alpha}, \alpha \in \mathbf{A}(\epsilon)$, and extension problems on their common zero sets, it is convenient to give a variation of the description of $E(\epsilon) \subset \Omega$, $0<\epsilon \leq \epsilon_{0}$. Recall that $\Omega_{0} \subset \Omega$ is a Stein neighborhood of 0 , and $Z_{\epsilon} \subset V \cap\left(D_{\epsilon} \times \Omega_{0}\right)$ denotes an irreducible subvariety containing $\operatorname{Graph}(f)$ and consisting of solutions $(z, \zeta)$ of functional equations $\left(\mathbf{I}_{w}\right), w \in D_{\epsilon}$, such that $\operatorname{dim}_{(z, f(z))}\left(Z_{\epsilon} \cap(\{z\} \times \Omega)\right)=1$ for a general point $z \in D_{\epsilon}$. For the normalization $v: \widetilde{Z}_{\epsilon} \rightarrow Z_{\epsilon}$, the extremal functions $h_{\alpha}$, 
$\alpha \in \mathbf{A}(\epsilon)$, were constructed using $\pi_{D} \circ v: \widetilde{Z}_{\epsilon} \rightarrow D_{\epsilon}$, where $\pi_{D}: D \times \Omega \rightarrow D$ and (later on) $\pi_{\Omega}: D \times \Omega \rightarrow \Omega$ denote the canonical projections. Write $\gamma=\pi_{D} \circ \nu$. Denote by $\mathcal{E}\left(Z_{\epsilon}\right) \subset \mathcal{E}(\epsilon)$ the subset of extremal functions thus obtained through $Z_{\epsilon}$ and by $E\left(Z_{\epsilon}\right) \subset$ $\Omega$ their common zero set. Write $\Gamma(\epsilon)$ for the set of all such $\gamma: \widetilde{Z}_{\epsilon} \rightarrow D_{\epsilon}$ and denote by $\left[Z_{\epsilon}\right]$ the member in $\Gamma(\epsilon)$ corresponding to the latter map. Then $\mathcal{E}(\epsilon)=\bigcup\left\{\mathcal{E}\left(Z_{\epsilon}\right)\right.$ : $\left.\left[Z_{\epsilon}\right] \in \Gamma(\epsilon)\right\}$ and $E(\epsilon)=\bigcap\left\{E\left(Z_{\epsilon}\right):\left[Z_{\epsilon}\right] \in \Gamma(\epsilon)\right\}$. The choice of the extremal functions $h_{\alpha} \in \mathcal{E}\left(Z_{\epsilon}\right)$ depends on the choice of one of the finitely many irreducible components $B_{j}$ of $v^{-1}\left(\operatorname{Graph}\left(\left.f\right|_{D_{\epsilon}}\right)\right)$. We denote by $\mathcal{E}\left(Z_{\epsilon}, B_{j}\right) \subset \mathcal{E}\left(Z_{\epsilon}\right)$ those arising from $B_{j}$, and by $E\left(Z_{\epsilon}, B_{j}\right) \subset \Omega$ the set of common zeros of $\mathcal{E}\left(Z_{\epsilon}, B_{j}\right)$. Clearly $E\left(Z_{\epsilon}\right)=\bigcap_{j} E\left(Z_{\epsilon}, B_{j}\right)$. We are ready to prove

Lemma 1.1.3. For $0<\epsilon_{2} \leq \epsilon_{1} \leq \epsilon_{0}$ we have $E\left(\epsilon_{2}\right) \subset E\left(\epsilon_{1}\right)$. Moreover, supposing that $\Omega^{\prime} \supset \Omega$ is a domain such that every $h \in \mathcal{E}\left(\epsilon_{1}\right) \cup \mathcal{E}\left(\epsilon_{2}\right)$ extends holomorphically to $\Omega^{\prime}$ and denoting by $E^{\prime}\left(\epsilon_{i}\right) \subset \Omega^{\prime}, i=1,2$, the common zero set of the extended functions $h^{\prime}$ on $\Omega^{\prime}$ of $h \in \mathcal{E}\left(\epsilon_{i}\right)$, we have $E^{\prime}\left(\epsilon_{2}\right) \subset E^{\prime}\left(\epsilon_{1}\right)$.

Proof. We continue with some generalities on $E\left(Z_{\epsilon}, B\right)$, where $0<\epsilon \leq \epsilon_{0}$, and $B=B_{j}$ is one of the irreducible branches of $v^{-1}\left(\operatorname{Graph}\left(\left.f\right|_{D_{\epsilon}}\right)\right)$. Since $B$ is a hypersurface in the normal complex space $\widetilde{Z}_{\epsilon}$, for some hypersurface $H \subset \widetilde{Z}_{\epsilon}$ such that $\operatorname{Sing}\left(\widetilde{Z}_{\epsilon}\right) \subset H$ and $B \not \subset H$, any $p \in B-H$ is a non-singular point of $B$ and $\gamma=\pi_{D} \circ v$ is a submersion at $p$. Denote by $T_{\widetilde{Z}_{\epsilon}}$ the tangent sheaf of $\widetilde{Z}_{\epsilon}$ and by $\mathcal{F} \subset T_{\widetilde{Z}_{\epsilon}}$ the relative tangent sheaf of $\gamma: \widetilde{Z}_{\epsilon} \rightarrow D_{\epsilon}$. Since $B$ is Stein, there is $\mu \in \Gamma(B, \mathcal{F}), \mu \not \equiv 0$. Write $\varphi=\pi_{\Omega} \circ \nu$. In particular, for $p \in B-H$, the fiber $\mathfrak{F}_{\gamma(p)}:=\gamma^{-1}(\gamma(p))$ of $\gamma: \widetilde{Z}_{\epsilon} \rightarrow D_{\epsilon}$ is smooth at $p \in \mathfrak{F}_{\gamma(p)}$. Suppose the restriction of $\varphi-f(\gamma(p))$ to $\mathfrak{F}_{\gamma(p)}$ vanishes exactly to the order $\ell-1$ at a general point of $B-H$. Let $X$ be a holomorphic vector field defined on some non-empty open set $V \subset \widetilde{Z}_{\epsilon}-H$ tangent to fibers $\mathfrak{F}_{\gamma(p)}$ such that $\left.\left.X\right|_{B \cap V} \equiv \mu\right|_{B \cap V}$. Since $\varphi-f(\gamma(p))$ vanishes on $\mathfrak{F}_{\gamma(p)}$ to the order $\ell-1$ at $p, X^{\ell} \varphi(p)$ is independent of the choice of $X \in \Gamma(V, \mathcal{F})$ extending $\left.\mu\right|_{B \cap V}$. Thus, there exists $\sigma \in \Gamma\left(B-H, \mathcal{O}^{N}\right)$ such that $\left.\sigma\right|_{B \cap V}=\left.X^{\ell} \varphi\right|_{B \cap V}$ for any such choices of $V$ and $X \in \Gamma(V, \mathcal{F})$. Since $\mathcal{F}$ is of rank 1 , for $\sigma^{\prime} \in \Gamma\left(B-H, \mathcal{O}^{N}\right)$ arising from any non-trivial section $\mu^{\prime} \in \Gamma(B, \mathcal{F})$, we must have $\mu^{\prime}=\lambda \mu$ for some non-trivial meromorphic function $\lambda$ on $B$, hence $\sigma^{\prime}=\lambda^{\ell} \sigma$ on $B-H$.

For $p \in B-H$, and $\sigma \in \Gamma\left(B-H, \mathcal{O}^{N}\right)$ as above, $\sigma(p)$ can be interpreted as an element $\tau(p) \in T_{\varphi(p)} \Omega \cong \mathbb{C}^{N}$. We thus have a holomorphic map $\tau: B-H-\operatorname{Zero}(\sigma) \rightarrow$ $T_{\Omega}^{\prime}$, and hence a separately real-analytic map $\mathfrak{A}: B-H-\operatorname{Zero}(\sigma) \rightarrow \mathfrak{X}(\Omega) \subset H^{2}(\Omega)$ given by $\mathfrak{A}(p)=h_{\tau(p)}=\mathfrak{h}(\cdot, \tau(p))$. Let $E^{\sigma} \subset \Omega$ be the common zero set of the extremal functions $\{\mathfrak{A}(p): p \in B-H-\operatorname{Zero}(\sigma)\}$. For $\sigma^{\prime}=\lambda^{\ell} \sigma$ as in the last paragraph, denoting by $\mathfrak{A}^{\prime}: B-H-\operatorname{Zero}\left(\sigma^{\prime}\right) \rightarrow \mathfrak{X}(\Omega)$ the analogue of $\mathfrak{A}$, the two extremal functions $\mathfrak{A}^{\prime}(p)$ and $\mathfrak{A}(p)$ are non-zero multiples of each other for $p$ belonging to the dense open subset $B-H-\operatorname{Zero}(\sigma)-\operatorname{Zero}\left(\sigma^{\prime}\right) \subset B$, hence $a$ priori the two closed subsets $E^{\sigma}, E^{\sigma^{\prime}} \subset \Omega$ are the same. In other words, $E^{\sigma}$ depends only on the rank-1 coherent subsheaf $\mathcal{F} \subset T_{\widetilde{Z}_{\epsilon}}$.

Consider any holomorphic deformation $\left\{f_{t}\right\}_{t \in \Delta}$ over $U \subset D_{\epsilon}$ constructed from $\left(Z_{\epsilon}, B\right)$ as in the proof of Proposition 1.1.2. There exists by construction $W \subset B$ such that $\left.\nu\right|_{W}: W \rightarrow U$ is a biholomorphism, so that, writing $\gamma(p)=z$ for $p \in W$, at a general point $z \in U$ we have $\eta(f(z))=\lambda(z) \tau(p)$ for some $\lambda(z) \in \mathbb{C}^{*}$. By Lemma 1.1.2 and 
by the Identity Theorem for real-analytic functions, the common zero set of the extremal functions $h_{\eta, z}, z \in U$, agrees with $E^{\sigma}$. Hence, $E^{\sigma}=E\left(Z_{\epsilon}, B\right)$.

We proceed now to prove $E\left(\epsilon_{2}\right) \subset E\left(\epsilon_{1}\right)$ whenever $0<\epsilon_{2} \leq \epsilon_{1} \leq \epsilon_{0}$. For $\left[Z_{\epsilon_{1}}\right] \in \Gamma\left(\epsilon_{1}\right)$, write $\left[\left.Z_{\epsilon_{1}}\right|_{D_{\epsilon}}\right] \in \Gamma\left(\epsilon_{2}\right)$ for the member obtained by restricting $\gamma$ : $\widetilde{Z}_{\epsilon_{1}} \rightarrow D_{\epsilon_{1}}$ to $D_{\epsilon_{2}}$, i.e., $\left.\gamma\right|_{\gamma^{-1}\left(D_{\epsilon_{2}}\right)}: \gamma^{-1}\left(D_{\epsilon_{2}}\right) \rightarrow D_{\epsilon_{2}}$. Let $B^{\prime}$ be an irreducible branch of $v^{-1}\left(\operatorname{Graph}\left(\left.f\right|_{D_{\epsilon_{2}}}\right)\right)$, and $B$ be that of $v^{-1}\left(\operatorname{Graph}\left(\left.f\right|_{D_{\epsilon_{1}}}\right)\right)$ containing $B^{\prime}$. Taking $\sigma_{1} \in \Gamma\left(B-H, \mathcal{O}^{N}\right)$ as above (replacing $\epsilon$ by $\epsilon_{1}$ and hence $\sigma$ by $\sigma_{1}$ ), we have $E\left(Z_{\epsilon_{1}}, B\right)=E^{\sigma_{1}}$ and $E\left(\left.Z_{\epsilon_{1}}\right|_{D_{\epsilon_{2}}}, B^{\prime}\right)=E^{\sigma_{2}}$, where $\sigma_{2}$ is the restriction of $\sigma_{1}$ to $B^{\prime}-H$. By Lemma 1.1.2 and the Identity Theorem we have $E^{\sigma_{2}}=E^{\sigma_{1}}$, hence $E\left(\left.Z_{\epsilon_{1}}\right|_{D_{\epsilon_{2}}}, B^{\prime}\right)=$ $E\left(Z_{\epsilon_{1}}, B\right)$. Finally, $E\left(\epsilon_{2}\right) \subset E\left(\epsilon_{1}\right)$ follows from $E(\epsilon)=\bigcap\left\{E\left(Z_{\epsilon}\right):\left[Z_{\epsilon}\right] \in \Gamma(\epsilon)\right\}$ and $E\left(Z_{\epsilon}\right)=\bigcap_{j} E\left(Z_{\epsilon}, B_{j}\right)$. Exactly the same argument gives the other statement in Lemma 1.1.3 when any $h \in \mathcal{E}\left(\epsilon_{1}\right) \cup \mathcal{E}\left(\epsilon_{2}\right)$ extends to $\Omega^{\prime} \supset \Omega$, as desired.

We are now ready to prove

Theorem 1.1.1. Let $D \Subset \mathbb{C}^{n}$ and $\Omega \Subset \mathbb{C}^{N}$ be bounded complete circular domains. Denote by $d s_{D}^{2}$, resp. $d s_{\Omega}^{2}$, the Bergman metric on $D$, resp. $\Omega$. Let $\lambda$ be any positive real number and $f:\left(D, \lambda d s_{D}^{2} ; 0\right) \rightarrow\left(\Omega, d s_{\Omega}^{2} ; 0\right)$ be a germ of holomorphic isometry. Then there exists an irreducible complex-analytic subvariety $S^{\sharp} \subset \mathbb{C}^{n} \times \mathbb{C}^{N}$ of dimension $n$ which contains the germ of $\operatorname{Graph}(f)$ at $(0,0)$.

Proof. Choose $\alpha \gg 1$ such that $D \Subset \alpha D_{\epsilon_{0}}=B^{n}\left(0 ; \alpha \epsilon_{0}\right)$. Let now $\epsilon^{\prime}>0$ be such that $\alpha \epsilon^{\prime}<\epsilon_{0}$. By Lemma 1.1.1, $\left.K_{D}\right|_{D \times D_{\epsilon^{\prime}}}$ extends holomorphically as a function of $(z, \bar{w})$ to $\alpha D_{\epsilon_{0}} \times D_{\epsilon^{\prime}}$ when we define

$$
K_{D}(\alpha z, w):=K_{D}(z, \alpha w)
$$

for $w \in D_{\epsilon^{\prime}}$. In particular, for each $w \in D_{\epsilon^{\prime}}$, the function $K_{D, w}(z)=K_{D}(z, w)$ extends holomorphically from $D$ to $B^{n}\left(0 ; \alpha \epsilon_{0}\right)$. Recall for $w \in D_{\epsilon^{\prime}}$ we have the functional equation

$$
\left(\mathbf{I}_{w}\right) \quad K_{\Omega}(\zeta, f(w))=A \cdot K_{D}(z, w)^{\lambda} .
$$

To proceed we make use of the proof of Proposition 1.1.2 and the notation adopted there.

Case 1: the functional equations are sufficiently non-degenerate. Consider first of all the case where $\left(\mathbf{I}_{w}\right), w \in D_{\epsilon}$, are sufficiently non-degenerate. On $D_{\epsilon_{0}} \times D_{\epsilon_{0}}$ the function $\log K_{D}(z, w)$ is well-defined and on the right-hand side of (2) the expression $K_{D}(z, w)^{\lambda}$ $:=e^{\lambda \log K_{D}(z, w)}$ is holomorphic in $(z, \bar{w})$, hence by (1) the same holds true for $(z, w) \in$ $\alpha D_{\epsilon_{0}} \times D_{\epsilon^{\prime}}$, noting that $D \Subset \alpha D_{\epsilon_{0}}=B^{n}\left(0 ; \alpha \epsilon_{0}\right)$. Recall that $V_{w} \subset D \times \Omega$ is the set of all $(z, \zeta) \in D \times \Omega$ satisfying $\left(\mathbf{I}_{w}\right), w \in D_{\epsilon}$, and that $V=\bigcap\left\{V_{w}: w \in D_{\epsilon^{\prime}}\right\}$ (noting that $\left.0<\epsilon^{\prime}<\epsilon_{0}\right)$. Recall that $0<\delta_{0}<1$ and $f\left(D_{\epsilon_{0}}\right) \Subset \Omega_{\delta_{0}} \Subset \Omega$ (cf. first and second paragraphs after Lemma 1.1.1). Choose now $\beta \gg 1$ such that $\Omega \Subset \beta \Omega_{\delta_{0}}=B^{N}\left(0 ; \beta \delta_{0}\right)$ and let $\delta>0$ be such that $\beta \delta<\delta_{0}$. Then, by Lemma 1.1.1, $K_{\Omega}(\zeta, \xi)$ is defined by extension for $\zeta \in \beta \Omega_{\delta_{0}}$ and $\xi \in \Omega_{\delta}$. Hence, for $w \in D_{\epsilon}$ the functional equation (2) is defined for $(z, \zeta) \in \alpha D_{\epsilon_{0}} \times \beta \Omega_{\delta_{0}}$. The set of all solutions $(z, \zeta) \in \alpha D_{\epsilon_{0}} \times \beta \Omega_{\delta_{0}}$ gives a subvariety $V^{\prime} \subset \alpha D_{\epsilon_{0}} \times \beta \Omega_{\delta_{0}}$ such that $V^{\prime} \cap(D \times \Omega)=V$. 
Let $k \geq 1$ be any positive integer. The function $K_{D, w}(z)$ can be extended holomorphically from $D$ to $B^{n}(0 ; k)=D_{k}$ whenever $|w|<k^{-1} \epsilon_{0}^{2}\left(<\epsilon_{0}\right)$. Likewise, letting $\ell \geq 1$ be any positive integer, the function $K_{\Omega, \xi}(\zeta)$ can be extended holomorphically from $\Omega$ to $B^{N}(0 ; \ell)=\Omega_{\ell}$ whenever $|\xi|<\delta_{\ell}:=\ell^{-1} \delta_{0}^{2}\left(<\delta_{0}\right)$. By the continuity of $f$ at 0 , for each $\ell \geq 1$ there exists $k(\ell)$ such that $f\left(D_{\epsilon_{\ell}}\right) \subset \Omega_{\delta_{\ell}}$ for $\epsilon_{\ell}:=k(\ell)^{-1} \epsilon_{0}^{2}$. We will choose $k(\ell)$ to be strictly increasing as $\ell \rightarrow \infty$. From the argument in the last paragraph we have irreducible subvarieties $V_{\ell}^{\sharp} \subset D_{k(\ell)} \times \Omega_{\ell}$ such that $V_{\ell}^{\sharp} \cap(D \times \Omega)=V$ for $\ell$ sufficiently large and such that for $\ell^{\prime}>\ell \geq 1$ we must have $V_{\ell^{\prime}}^{\sharp} \cap\left(D_{k(\ell)} \times \Omega_{\ell}\right)=V_{\ell}^{\sharp}$, by the Identity Theorem for holomorphic functions. Since $k(\ell) \rightarrow \infty$ as $\ell \rightarrow \infty$, writing $V^{\sharp}:=\bigcup_{\ell>1} V_{\ell}^{\sharp}$, we have obtained a subvariety $V^{\sharp} \subset \mathbb{C}^{n} \times \mathbb{C}^{N}$ such that $V^{\sharp} \cap(D \times \Omega)=V$ and $V^{\sharp} \cap\left(D_{k(\ell)} \times \Omega_{\ell}\right)=V_{\ell}^{\sharp}$ for each positive integer $\ell$. When the system of functional equations $\left(\mathbf{I}_{w}\right), w \in D_{\epsilon}$, is sufficiently non-degenerate, it suffices to take $S^{\sharp}$ to be the irreducible component of $V^{\sharp}$ containing $\operatorname{Graph}(f)$, so that $\operatorname{dim}\left(S^{\sharp}\right)=n=\operatorname{dim}(\operatorname{Graph}(f))$, and $S^{\sharp} \subset \mathbb{C}^{n} \times \mathbb{C}^{N}$ extends $\operatorname{Graph}(f)$ as a subvariety.

Case 2: the functional equations are not sufficiently non-degenerate. For $0<\epsilon \leq \epsilon_{0}$ we define $\widehat{\mathcal{E}}(\epsilon):=\bigcup\{\mathcal{E}(\beta): 0<\beta \leq \epsilon\}$, and write $\widehat{E}(\epsilon) \subset \Omega$ for the common zero set of $\widehat{\mathcal{E}}(\epsilon)$. Thus, $\widehat{E}(\epsilon)=\bigcap\{E(\beta): 0<\beta \leq \epsilon\}$. Obviously, $\widehat{E}(\epsilon) \supset \widehat{E}\left(\epsilon_{0}\right)$ whenever $0<\epsilon \leq \epsilon_{0}$. By Lemma 1.1.3, we have $E\left(\epsilon_{2}\right) \subset E\left(\epsilon_{1}\right)$ whenever $0<\epsilon_{2} \leq \epsilon_{1} \leq \epsilon_{0}$, hence $\widehat{E}(\epsilon) \subset \widehat{E}\left(\epsilon_{0}\right)$. Thus $\widehat{E}(\epsilon)=\widehat{E}\left(\epsilon_{0}\right):=\widehat{E}$ whenever $0<\epsilon \leq \epsilon_{0}$.

From Proposition 1.1.2, for $0<\epsilon \leq \epsilon_{0}$ we have $\operatorname{Graph}(f) \subset V \cap(D \times E(\epsilon))$, hence $\operatorname{Graph}(f) \subset V \cap(D \times \widehat{E})$. Recall that there exists an increasing sequence $k(\ell), 1 \leq$ $\ell<\infty$, of positive integers such that $f\left(D_{\epsilon_{\ell}}\right) \subset \Omega_{\delta_{\ell}}$ for $\epsilon_{\ell}:=k(\ell)^{-1} \epsilon_{0}^{2}$ and $\delta_{\ell}=\ell^{-1} \delta_{0}^{2}$. By Lemma 1.1.2, any $h_{\alpha} \in \widehat{\mathcal{E}}\left(\epsilon_{\ell}\right)$ is definable on $\Omega_{\ell}$, with common zero set on $\Omega_{\ell}$ to be denoted by $\widehat{E}_{\ell}^{\sharp} \subset \Omega_{\ell}$. By Lemma 1.1 .3 (cf. last paragraph), $\bigcup_{\ell} \widehat{E}_{\ell}^{\sharp}:=\widehat{E}^{\sharp} \subset \mathbb{C}^{N}$ is a subvariety such that $\widehat{E}^{\sharp} \cap \Omega=\widehat{E}$. Define now $T^{\sharp}:=V^{\sharp} \cap\left(\mathbb{C}^{n} \times \widehat{E}^{\sharp}\right) \supset \operatorname{Graph}(f)$. Then the unique irreducible component $S^{\sharp}$ of $T^{\sharp}$ containing $\operatorname{Graph}(f)$ extends the latter as a subvariety, as desired.

\subsection{Holomorphic isometric embeddings defined by extensions of germs of graphs}

Let $f:\left(D, \lambda d s_{D}^{2} ; 0\right) \rightarrow\left(\Omega, d s_{\Omega}^{2} ; 0\right)$ be a germ of holomorphic isometry at 0 between bounded complete circular domains, with $f(0)=0$, and $S \subset D \times \Omega$ be the extension of $\operatorname{Graph}(f)$ to $D \times \Omega$ as a complex-analytic subvariety. For the study of properties of $S$ we will need the following well-known lemma resulting from the Cauchy-Schwarz inequality.

Lemma 1.2.1. Let $G$ be a bounded domain and denote by $K_{G}(z, w)$ its Bergman kernel. Then for any $z, w \in G$ we have $\left|K_{G}(z, w)\right|^{2} \leq K_{G}(z, z) K_{G}(w, w)$. Moreover, equality holds if and only if $z=w$.

Proof. Let $\left(g_{j}\right)_{j=0}^{\infty}$ be an orthonormal basis of the Hilbert space $H^{2}(G)$ of square-integrable holomorphic functions on $G$. Then $K_{G}(z, w)=\sum_{j=0}^{\infty} g_{j}(z) \overline{g_{j}(w)}$, and the in- 
equality $\left|K_{G}(z, w)\right|^{2} \leq K_{G}(z, z) K_{G}(w, w)$ results from the Cauchy-Schwarz inequality for the Hilbert space $\mathbb{H}$ of square-integrable sequences of complex numbers. Writing $\Psi(z)=\left(g_{0}(z), g_{2}(z), \ldots\right)$, equality holds if and only if $\Psi(z)=\alpha \Psi(w)$ for some complex number $\alpha$. From the reproducing property of $K_{G}(z, w)$ this is the case if and only if $g(z)=\alpha g(w)$ for any $g \in H^{2}(G)$, which obviously holds true if and only if $z=w$.

Under some mild conditions we have a sharpened result on interior extension.

Theorem 1.2.1. Let $D$ and $\Omega$ be bounded complete circular domains, $\lambda$ be any positive real number, and $f:\left(D, \lambda d s_{D}^{2} ; 0\right) \rightarrow\left(\Omega, d s_{\Omega}^{2} ; 0\right)$ be a germ of holomorphic isometry. Then $\operatorname{Graph}(f) \subset D \times \Omega$ extends to a complex-analytic subvariety $S \subset D \times \Omega$ which is the graph of a holomorphic isometry $F:\left(D^{\prime},\left.\lambda d s_{D}^{2}\right|_{D^{\prime}}\right) \rightarrow\left(\Omega, d s_{\Omega}^{2}\right)$ for some connected open subset $D^{\prime} \subset D$ containing $D_{\epsilon}$. Suppose $\varphi_{\Omega}(\zeta):=K_{\Omega}(\zeta, \zeta)$ is an exhaustion function on $\Omega$. Then $D=D^{\prime}$ and $F:\left(D, \lambda d s_{D}^{2}\right) \rightarrow\left(\Omega, d s_{\Omega}^{2}\right)$ is a holomorphic isometry. If furthermore $\varphi_{D}(z):=K_{D}(z, z)$ is an exhaustion function on $D$, then $F$ is proper.

Proof. By Theorem 1.1.1, Graph $(f)$ extends analytically to an irreducible subvariety $S \subset$ $D \times \Omega$. Let $\rho_{D}: S \rightarrow D$ and $\rho_{\Omega}: S \rightarrow \Omega$ be the canonical projections. By definition the real-analytic identity $(\dagger) \lambda \rho_{D}^{*}\left(d s_{D}^{2}\right)=\rho_{\Omega}^{*}\left(d s_{\Omega}^{2}\right)$ holds true on $\operatorname{Graph}(f)$, hence on $\operatorname{Reg}(S)$ by analytic continuation. We claim that for any $p \in D$, the fiber $\Phi_{p}:=\rho_{D}^{-1}(p)$ is 0 dimensional. Suppose otherwise. Let $\Phi_{p}$ be a positive-dimensional fiber and $(p, q) \in \Phi_{p}$ be a smooth point belonging to an irreducible branch of positive dimension. Let $\eta=$ $\left(\eta^{\prime}, \eta^{\prime \prime}\right)$ be a non-zero real vector tangent to $\Phi_{p}$ at $(p, q)$. Then $\eta^{\prime}=0, \eta^{\prime \prime} \neq 0$. Thus, $\rho_{D}^{*}\left(d s_{D}^{2}\right)(\eta, \eta)=0$ while $\rho_{\Omega}^{*}\left(d s_{\Omega}^{2}\right)(\eta, \eta)=d s_{\Omega}^{2}\left(\eta^{\prime \prime}, \eta^{\prime \prime}\right)>0$. If $(p, q) \in \operatorname{Reg}(S)$, then we have reached a contradiction since $(\dagger)$ holds true on $\operatorname{Reg}(S)$.

In general, let $\mathcal{I} \subset \mathcal{O}_{D \times \Omega}$ be the ideal sheaf of $S \subset D \times \Omega$, and let $\mathcal{F} \subset \mathcal{O}\left(\left.T_{D \times \Omega}\right|_{S}\right)$ be the coherent sheaf on $S$ whose stalk at $s \in S$ consists of all $\xi \in \mathcal{O}_{s}\left(T_{D \times \Omega}\right)$ such that $\xi f=0$ for every $f \in \mathcal{I}_{s}$. Then there exists $\xi \in \mathcal{F}_{(p, q)}$ such that $\operatorname{Re} \xi(p, q)=\eta$. Thus, writing $\xi=\left(\xi^{\prime}, \xi^{\prime \prime}\right)$, by analytic continuation the germ of function $\lambda \rho_{D}^{*} d s_{D}^{2}\left(\operatorname{Re} \xi^{\prime}, \operatorname{Re} \xi^{\prime}\right)-\rho_{\Omega}^{*} d s_{\Omega}^{2}\left(\operatorname{Re} \xi^{\prime \prime}, \operatorname{Re} \xi^{\prime \prime}\right)$ at $(p, q)$ vanishes, which is a contradiction since $\operatorname{Re} \xi^{\prime}(p, q)=\eta^{\prime}=0$ and $\operatorname{Re} \xi^{\prime \prime}(p, q)=\eta^{\prime \prime} \neq 0$.

Denote by $B \subsetneq S$ the subvariety over which $\rho_{D}$ fails to be a local biholomorphism. Then $S-B$ is locally the graph of a holomorphic isometry between open subsets of $D$ and $\Omega$ with respect to restrictions of the Kähler metrics $\lambda d s_{D}^{2}$ and $d s_{\Omega}^{2}$. Since $\rho_{D}: S \rightarrow D$ is a local biholomorphism at a general point and its fibers are 0-dimensional, it is an open map. We claim that $\rho_{D}: S \rightarrow D$ is injective. Suppose otherwise. By the openness of $\rho_{D}$, there exists $x \in D$ and two distinct points $y_{1}, y_{2} \in \Omega$ such that $\left(x, y_{1}\right),\left(x, y_{2}\right) \in S-B$. Thus, there exist some simply connected neighborhoods $U$ of $x$ and $W_{1}$, resp. $W_{2}$, of $\left(x, y_{1}\right)$, resp. $\left(x, y_{2}\right)$, such that $\left.\rho_{D}\right|_{W_{1}}: W_{1} \cong U$ and $\left.\rho_{D}\right|_{W_{2}}: W_{2} \cong U$ are biholomorphisms. For $z \in U$ and $i=1,2$ we describe $W_{i}$ as the graph of $f_{i}: U \rightarrow \Omega$, which is a holomorphic isometry with respect to $\left.\lambda d s_{D}^{2}\right|_{U}$ and $d s_{\Omega}^{2}$. Recall that $K_{D}(z, 0)$ is a positive constant $C$. By Lemma 1.1.1, shrinking $\epsilon_{0}>0$ if necessary we may assume that $\operatorname{Re} K_{D}(z, w)>0$ for any $(z, w) \in D \times D_{\epsilon_{0}}$, so that $K_{D}(z, w)^{\lambda}$ is defined as a function holomorphic in $(z, \bar{w})$ for $(z, w) \in D \times D_{\epsilon_{0}}$. By Proposition 1.1.2 $K_{\Omega}(f(z), f(w))-A \cdot K_{D}(z, w)^{\lambda}=0$ for $z, w \in D_{\epsilon_{0}}$. Thus, by analytic continuation 
$K_{\Omega}(y, f(w))-A \cdot K_{D}(x, w)^{\lambda}=0$ holds true for $w \in D_{\epsilon_{0}}$ and for any $(x, y) \in S$. In particular, we have

$$
K_{\Omega}\left(y_{1}, f(w)\right)=A \cdot K_{D}(x, w)^{\lambda}=K_{\Omega}\left(y_{2}, f(w)\right) .
$$

Since $x \in U$ is arbitrary, we conclude that

$$
K_{\Omega}\left(f_{1}(z), f(w)\right)=K_{\Omega}\left(f_{2}(z), f(w)\right)
$$

for any $(z, w) \in U \times D_{\epsilon_{0}}$. Fix an arbitrary point $z \in U$. Consider $\psi: \Omega \rightarrow \mathbb{C}$ defined by $\psi(\xi)=K_{\Omega}\left(\xi, f_{1}(z)\right)-K_{\Omega}\left(\xi, f_{2}(z)\right)$. Define furthermore $s: S \rightarrow \mathbb{C}$ by $s(x, y)=$ $\psi(y)$ for $(x, y) \in S$. By (1) we have $s(w, f(w))=0$ whenever $w \in D_{\epsilon_{0}}$. From the irreducibility of $S$, we deduce by analytic continuation that $s \equiv 0$ on $S$. In particular, substituting $(x, y)=\left(z, f_{i}(z)\right) \in S-B, i=1,2$, we conclude from $s\left(z, f_{1}(z)\right)=$ $s\left(z, f_{2}(z)\right)=0$ that

$$
K_{\Omega}\left(f_{1}(z), f_{1}(z)\right)=K_{\Omega}\left(f_{1}(z), f_{2}(z)\right), \quad K_{\Omega}\left(f_{2}(z), f_{1}(z)\right)=K_{\Omega}\left(f_{2}(z), f_{2}(z)\right)
$$

for any $z \in U$. Thus, $K\left(f_{1}(z), f_{2}(z)\right)$ is real and we have

$$
K_{\Omega}\left(f_{1}(z), f_{2}(z)\right)=K_{\Omega}\left(f_{1}(z), f_{1}(z)\right)=K_{\Omega}\left(f_{2}(z), f_{2}(z)\right) .
$$

From Lemma 1.1.2 we have

$$
\left|K_{\Omega}\left(f_{1}(z), f_{2}(z)\right)\right|^{2} \leq K_{\Omega}\left(f_{1}(z), f_{1}(z)\right) K_{\Omega}\left(f_{2}(z), f_{2}(z)\right)
$$

and equality holds if and only if $f_{1}(z)=f_{2}(z)$. Thus, (2) implies that $f_{1}(z)=f_{2}(z)$ for $z \in U$, proving that each fiber of $\rho_{D}: S \rightarrow D$ consists of at most one point. Hence, $S$ is the graph of some holomorphic map $F: D^{\prime} \rightarrow \Omega$ defined on some neighborhood $D^{\prime} \subset D$ of 0 containing $D_{\epsilon_{0}}$. To prove that $F$ is injective let $z_{1}, z_{2} \in D^{\prime}$ be such that $F\left(z_{1}\right)=F\left(z_{2}\right)$. For $w \in D_{\epsilon_{0}}$,

$$
K_{D}\left(z_{1}, w\right)^{\lambda}=A^{-1} K_{\Omega}\left(F\left(z_{1}\right), f(w)\right)=A^{-1} K_{\Omega}\left(F\left(z_{2}\right), f(w)\right)=K_{D}\left(z_{2}, w\right)^{\lambda} .
$$

Since $K_{D}(z, 0)=A$ is positive, (3) implies that for some $\epsilon$ sufficiently small, $0<\epsilon \leq \epsilon_{0}$,

$$
K_{D}\left(z_{1}, w\right)=K_{D}\left(z_{2}, w\right)
$$

whenever $w \in D_{\epsilon_{0}}$, hence for any $w \in D$ by the Identity Theorem. By the reproducing property of $K_{D}(z, w), h\left(z_{1}\right)=h\left(z_{2}\right)$ for any $h \in H^{2}(D)$, hence $z_{1}=z_{2}$, i.e., $F$ is injective.

Assume now $\varphi_{\Omega}(\zeta):=K_{\Omega}(\zeta, \zeta)$ is an exhaustion function. Suppose $D^{\prime} \subsetneq D$ and let $p \in \partial D^{\prime} \cap D$. From the functional equations $\left(\mathbf{I}_{w}\right), w \in D_{\epsilon_{0}}$, we have $K_{\Omega}(F(z), F(z))=$ $A \cdot K_{D}(z, z)^{\lambda}$. Since $K_{\Omega}(\zeta, \zeta)$ is an exhaustion function of $\zeta$, any limit point $(p, q)$ of points $(z, F(z))$ as $z$ approaches $p$ must lie in $D \times \Omega$, i.e., $q \in \Omega$. Since $S \subset D \times \Omega$ is a subvariety, in particular closed, it follows that $(p, q) \in S$, so that $S$ is the graph of some holomorphic map in a neighborhood of $(p, q) \in S$, so that $p \in D^{\prime}$, a plain contradiction. We conclude that $D^{\prime}=D$, i.e., $F: D \rightarrow \Omega$ is a global holomorphic isometry. 
Finally, assume $\varphi_{D}(z)=K_{D}(z, z)$ is an exhaustion function. Then, for any discrete sequence of points $\left(z_{m}\right)_{m=0}^{\infty}$ on $D, K_{D}\left(z_{m}, z_{m}\right)$ must diverge to $\infty$ as $n \rightarrow \infty$. Hence, $K_{\Omega}\left(F\left(z_{m}\right), F\left(z_{m}\right)\right)=A \cdot K_{D}\left(z_{m}, z_{m}\right)^{\lambda}$ must also diverge to $\infty$, implying that $\left(F\left(z_{m}\right)\right)_{m=0}^{\infty}$ is discrete. As a consequence, $F: D \rightarrow \Omega$ must be proper, as desired.

Remarks. For bounded complete circular domains $D_{1}$ and $D_{2}$, a biholomorphism $\Phi$ : $\left(D_{1} ; 0\right) \rightarrow\left(D_{2}, 0\right)$ must be linear, by a result of H. Cartan's (cf. Mok [Mk2, Chap. 4, §2, Thm. 1]). Thus, the exhaustive property of $\varphi_{D}(z)$ is a property of $(D ; 0)$ independent of its realization as a bounded complete circular domain marked at 0 .

From the proof of Theorem 1.2.1 we deduce

Corollary 1.2.1. In the notation of the proof of Theorem 1.1 .1 , let $S^{\sharp} \subset \mathbb{C}^{n} \times \mathbb{C}^{N}$ be the irreducible component of $V^{\sharp} \cap\left(\mathbb{C}^{n} \times \widehat{E}^{\sharp}\right)$ containing $\operatorname{Graph}(f)$. Suppose the function $\varphi_{\Omega}=K_{\Omega}(\zeta, \zeta)$ is an exhaustion function on $\Omega$. Then $S^{\sharp} \cap(D \times \Omega)$ is irreducible. In other words, denoting by $S$ the irreducible component of $V \cap(D \times \widehat{E})$ containing $\operatorname{Graph}(f)$, we have $S^{\sharp} \cap(D \times \Omega)=S$.

Proof. By Theorem 1.2.1, $S$ is the graph of $F:\left(D, \lambda d s_{D}^{2}\right) \rightarrow\left(\Omega, d s_{\Omega}^{2}\right)$. Suppose over some non-empty open subset $U \subset D \times \Omega$ there are two branches of $S^{\sharp} \cap(D \times \Omega)$ described by $\left(z, f_{1}(z)\right)$ and $\left(z, f_{2}(z)\right)$, where $f_{i}: U \rightarrow \Omega, i=1,2$, are holomorphic maps. The argument of analytic continuation leading to the identities $K_{\Omega}\left(f_{1}(z), f_{2}(z)\right)=$ $K_{\Omega}\left(f_{1}(z), f_{1}(z)\right)=K_{\Omega}\left(f_{2}(z), f_{2}(z)\right)$ remains valid. To conclude it suffices to note that the argument using the Cauchy-Schwarz inequality on $\Phi(\Omega) \subset \mathbb{H}$, which gives $f_{1}(z)=$ $f_{2}(z)$ once the identities are established, remains applicable since both $f_{1}(z)$ and $f_{2}(z)$ lie on $\Omega$.

As will be seen in 3.2, there exist non-standard holomorphic isometric embeddings of the Poincaré disk into polydisks. In such an example Graph $(f)$ extends to an affine-algebraic variety $S^{\sharp}$, but $S^{\sharp}$ is no longer the graph of a 'univalent' map.

\subsection{Holomorphic isometric embeddings between bounded symmetric domains}

In 2003, Clozel-Ullmo proved an extension theorem for germs of holomorphic isometries up to integral normalizing constants from the unit disk into the polydisk equipped with the Bergman metric, showing that any such germ of map extends to a holomorphic isometric immersion on the unit disk and that moreover its graph extends to an affine-algebraic variety. This was a crucial step in the proof of the total geodesy of such germs of holomorphic isometries arising from some special algebraic correspondences in [CU]. (For a discussion on methods of analytic continuation in relation to [CU], cf. Mok [Mk5, (2.2) and §4].) For germs of holomorphic isometries between bounded symmetric domains in general, applications of Theorem 1.1.1 and Theorem 1.2.1 and their proofs yield

Theorem 1.3.1. Let $D \Subset \mathbb{C}^{n}$ and $\Omega \Subset \mathbb{C}^{N}$ be bounded symmetric domains in their Harish-Chandra realizations. Let $\lambda$ be any positive real number and $f:\left(D, \lambda d s_{D}^{2} ; 0\right) \rightarrow$ $\left(\Omega, d s_{\Omega}^{2} ; 0\right)$ be a germ of holomorphic isometry at $0 \in D$. Then the germ $\operatorname{Graph}(f)$ at 
$(0,0)$ extends to an affine-algebraic subvariety $S^{\sharp} \subset \mathbb{C}^{n} \times \mathbb{C}^{N}$ such that $S:=S^{\sharp} \cap(D \times \Omega)$ is the graph of a proper holomorphic isometric embedding $F:\left(D, \lambda d s_{D}^{2}\right) \rightarrow\left(\Omega, d s_{\Omega}^{2}\right)$.

For the proof of Theorem 1.3.1 we will make use of specific forms of Bergman kernels on bounded symmetric domains as given by the following well-known lemma.

Lemma 1.3.1. Let $G \Subset \mathbb{C}^{m}$ be an irreducible bounded symmetric domain in its HarishChandra realization, and denote by $K_{G}(z, w)$ its Bergman kernel. Then $K_{G}(z, w)=$ $1 / Q_{G}(z, w)$, where $Q_{G}$ is a polynomial in $\left(z_{1}, \ldots, z_{m}, \overline{w_{1}}, \ldots, \overline{w_{m}}\right)$ such that $Q_{G}(z, z)$ $>0$ on $G$ and $Q_{G}(z, z)=0$ for $z \in \partial G$.

We have more precisely $Q_{G}(z, w)=h_{G}(z, w)^{p_{G}}$, where $h_{G}(z, w)$ is some polynomial in $\left(z_{1}, \ldots, z_{m}, \overline{w_{1}}, \ldots, \overline{w_{m}}\right)$ and $p_{G}$ is a positive integer depending on $G$. The polynomial $h_{G}(z, w)$ in $(z, \bar{w})$ is characterized by the property $(\dagger)$ to be specified below (cf. Faraut-Korányi [FK, pp. 76-77]). Denote by $r$ the rank of $G$ as a bounded symmetric domain. The isotropy subgroup $K$ of $\operatorname{Aut}_{0}(G)$ acts as a group of $G$-preserving unitary transformations on the Euclidean space $\mathbb{C}^{m}$. Using Harish-Chandra coordinates, for each maximal polydisk $P \cong \Delta^{r}$ on $G$ passing through 0 there exists $\gamma \in K$ such that $\gamma(P)$ is the unit polydisk $\Pi=\Delta^{r} \times\{0\}$. Each $z \in G$ is contained in a maximal polydisk $P \subset G$, hence there exists $\gamma \in K$ such that $\gamma(z)=\left(a_{1}, \ldots, a_{r} ; 0\right) \in \Pi$. For some positive constant $\alpha_{G}$ the polynomial $h_{G}(z, w)$ in $(z, \bar{w})$ is characterized by the property $(\dagger) h_{G}(z, z)=\alpha_{G}\left(1-\left|a_{1}\right|^{2}\right) \times \cdots \times\left(1-\left|a_{r}\right|^{2}\right)$. As examples, in the case of type-I domains $D_{p, q}^{\mathrm{I}}$ in the complex Euclidean space $M(p, q)$ of $p$-by- $q$ matrices with complex entries defined by $D_{p, q}^{\mathrm{I}}:=\left\{Z \in M(p, q): I-\bar{Z}^{t} Z>0\right\}$, the Bergman kernel is given by $K_{D_{p, q}^{\mathrm{I}}}(Z, W)=\alpha_{p, q} \cdot \operatorname{det}\left(I-\bar{W}^{t} Z\right)^{-(p+q)}$ for some positive constant $\alpha_{p, q}$ (cf. Mok [Mk2, Chap. 4, p. $80 \mathrm{ff}$.] for this and other classical domains).

Proof of Theorem 1.3.1. Recall the functional equations $\left(\mathbf{I}_{w}\right), w \in D_{\epsilon}$, in Proposition 1.1.2, arising from a germ of holomorphic isometry $f:\left(D, \lambda d s_{D}^{2} ; 0\right) \rightarrow\left(\Omega, d s_{\Omega}^{2} ; 0\right)$, where $f$ is assumed to be defined on $D_{\epsilon_{0}}=B^{n}\left(0 ; \epsilon_{0}\right) \Subset \mathbb{C}^{n}$. It may happen a priori that the normalizing constant $\lambda$ is irrational (cf. Proposition 3.1.2). The functions $\varphi_{D}(z)=$ $K_{D}(z, z)$ and $\varphi_{\Omega}(\zeta)=K_{\Omega}(\zeta, \zeta)$ are exhaustion functions by Lemma 1.3.1. Thus, by Theorem 1.2.1, $f$ extends to a proper holomorphic map $F:\left(D, \lambda d s_{D}^{2}\right) \rightarrow\left(\Omega, d s_{\Omega}^{2}\right)$ such that $\operatorname{Graph}(F) \subset D \times \Omega$ extends to a complex-analytic subvariety $S^{\sharp} \subset \mathbb{C}^{n} \times \mathbb{C}^{N}$. By the fine structure of the boundary of bounded symmetric domains in their Harish-Chandra realizations (cf. Wolf [Wo]), there is a decomposition of $\partial D$ into a finite union of orbits under $\operatorname{Aut}_{0}(D)$. The set $\operatorname{Reg}(\partial D)$ of regular points of $\partial D$ is a locally closed real-analytic submanifold of $\mathbb{C}^{n}$ which is dense in $\partial D$. The preceding discussion holds analogously for the bounded symmetric domain $\Omega \Subset \mathbb{C}^{N}$ in its Harish-Chandra realization.

We claim that $\lambda$ must be a rational number. Since $\operatorname{Graph}(F)$ extends to a subvariety $S^{\sharp} \subset \mathbb{C}^{n} \times \mathbb{C}^{N}$, for a general point $b \in \operatorname{Reg}(\partial D)$, there is a neighborhood $U_{b}$ of $b$ in $\mathbb{C}^{n}$ and a holomorphic map $F^{b}: U_{b} \rightarrow \mathbb{C}^{N}$ such that $\left.F^{b}\right|_{U_{b} \cap D}$ agrees with $\left.F\right|_{U_{b} \cap D}$. We have

$$
K_{\Omega}\left(F^{\mathrm{b}}(z), F^{\mathrm{b}}(z)\right)=A \cdot K_{D}(z, z)^{\lambda}
$$


for $z, w \in U_{b} \cap D$. By Lemma 1.3.1 we have

$$
A \cdot Q_{\Omega}\left(F^{b}(z), F^{b}(z)\right)=Q_{D}(z, z)^{\lambda}
$$

for $z \in U_{b} \cap D$. Write $\rho_{D}(z)=-h_{D}(z, z)$ on $\mathbb{C}^{n}$ and $\rho_{\Omega}(\zeta)=-h_{\Omega}(\zeta, \zeta)$ on $\mathbb{C}^{N}$. On $U_{b}$ the function $\sigma(z)=\rho_{\Omega}\left(F^{b}(z)\right)$ is real-analytic. We have $\sigma<0$ on $U_{b} \cap D$ and $\sigma=0$ on $U_{b} \cap \partial D$. The function $\rho_{D}$, resp. $\rho_{\Omega}$, vanishes to order 1 along $\operatorname{Reg}(\partial D), \operatorname{resp} . \operatorname{Reg}(\partial \Omega)$. Letting $\ell \geq 1$ be the vanishing order of $\sigma$ along $U_{b} \cap \partial \Omega$, by equating vanishing orders on both sides of (1) we conclude that $\ell p_{\Omega}=\lambda p_{D}$, hence $\lambda=\ell p_{\Omega} / p_{D}$ is a rational number, as claimed.

Write now $\lambda=p / q$, where $p$ and $q$ are positive integers. We adopt the notation in the proof of Theorem 1.1.1. There we have a subvariety $V^{\sharp} \subset \mathbb{C}^{n} \times \mathbb{C}^{N}$, a subvariety $\widehat{E}^{\sharp} \subset \mathbb{C}^{N}$ such that $T^{\sharp}=V^{\sharp} \cap\left(\mathbb{C}^{n} \times \widehat{E}^{\sharp}\right)$ contains Graph $(f), T^{\sharp}$ is irreducible and of dimension $n$ at a general point of $\operatorname{Graph}(f)$, and $S^{\sharp} \subset \mathbb{C}^{n} \times \mathbb{C}^{N}$ is the unique irreducible component of $T^{\sharp}$ containing $\operatorname{Graph}(f)$. In the current situation where $\lambda=p / q$ is rational, let $W^{\sharp}$ be the set of common solutions $(z, \zeta)$ on $\mathbb{C}^{n} \times \mathbb{C}^{N}$ to the equations $K_{\Omega}(\zeta, f(w))^{q}=A \cdot K_{D}(z, w)^{p}$ as $w$ ranges over some $D_{\epsilon_{0}}=B^{n}\left(0 ; \epsilon_{0}\right)$. Then $V^{\sharp} \subset W^{\sharp}$ and the germs of $V^{\sharp}$ and $W^{\sharp}$ at $(0,0)$ agree with each other. By Lemma 1.3.1, the functions $K_{D, w}(z)=K_{D}(z, w)$ and $K_{\Omega, \xi}=K_{\Omega}(\zeta, \xi)$ are rational functions, hence $W^{\sharp} \subset \mathbb{C}^{n} \times \mathbb{C}^{N}$ is affine-algebraic. The subvariety $\widehat{E}=\widehat{E}\left(\epsilon_{0}\right) \subset \Omega$ is defined by extremal functions $\left\{h_{\alpha}\right\}_{\alpha \in \mathbf{A}(\beta)}, 0<\beta \leq \epsilon_{0}$, and $\widehat{E}=\widehat{E}^{\sharp} \cap \Omega$. By the formula in Lemma 1.1.2 expressing $h_{\alpha}=h_{\eta, z_{0}}$ in terms of $K_{\Omega}$, it follows that each $h_{\alpha}$ is a rational function. Thus $\widehat{E}=H \cap \Omega$ for some affine-algebraic variety $H \subset \mathbb{C}^{N}$. Finally, $S^{\sharp}$ is equivalently the irreducible component of $W^{\sharp} \cap\left(\mathbb{C}^{n} \times \widehat{H}\right)$ containing $\operatorname{Graph}(f)$, hence also affinealgebraic, as desired.

When $D$ is the unit disk $\Delta$, and $F:\left(\Delta, \lambda d s_{\Delta}^{2}\right) \rightarrow\left(\Omega, d s_{\Omega}^{2}\right)$ is a holomorphic isometry, by Theorem 1.3.1, $F$ is a proper holomorphic isometric embedding, and $S:=\operatorname{Graph}(F)$ extends as a subvariety to an affine-algebraic subvariety $S^{\sharp} \subset \mathbb{C} \times \mathbb{C}^{N}$. It follows in particular that $F: \Delta \rightarrow \Omega$ extends to a continuous mapping $F^{b}: \bar{\Delta} \rightarrow \bar{\Omega}$. For a general point $b \in \partial \Delta$, there is a neighborhood $U_{b}$ of $b$ on $\mathbb{C}$ such that $\left.F\right|_{U_{b} \cap \Delta}$ extends holomorphically to $U_{b}$. When the latter fails to be the case, $b$ will be called a singular point of $F$, and we will say that $b$ lies over the branch point $F^{b}(b) \in \partial \Omega$.

Germs of holomorphic isometries up to normalizing constants between bounded symmetric domains equipped with the Bergman metric may fail to be totally geodesic (cf. 3.2 and 3.3). In view of such examples we pose the question of finding conditions under which germs of holomorphic isometries are necessarily totally geodesic. In the case where the domain is irreducible and of rank $\geq 2$, as observed by Clozel-Ullmo [CU], total geodesy follows from the proof of Hermitian metric rigidity of Mok [Mk1, Mk2]. Mok [Mk3] proved an analogue on algebraic extension for germs of holomorphic isometries up to integral normalizing constants from an $n$-ball to a product of $n$-balls under a certain non-degeneracy assumption, showing in the case of $n \geq 2$ that any such map must necessarily be totally geodesic by applying Alexander's Theorem [Al]. Using Theorem 1.3.1, the latter result can be improved by removing the non-degeneracy assumption and by allowing the normalizing constant $\lambda$ to be a priori any positive real number. Regarding the characterization of totally geodesic maps among holomorphic isometries we now have 
Theorem 1.3.2. Let $D \Subset \mathbb{C}^{n}$ and $\Omega \Subset \mathbb{C}^{N}$ be bounded symmetric domains, $\lambda>0$, and $f:\left(D, \lambda d s_{D}^{2} ; 0\right) \rightarrow\left(\Omega, d s_{\Omega}^{2} ; 0\right)$ be a germ of holomorphic isometry. Then $f$ extends to a totally geodesic holomorphic embedding $F:\left(D, \lambda d s_{D}^{2}\right) \rightarrow\left(\Omega, d s_{\Omega}^{2}\right)$

(a) whenever each irreducible component of $D$ is of rank $\geq 2$;

(b) whenever $D$ is of rank 1 and dimension $\geq 2$, i.e., $D \cong B^{n}, n \geq 2$, and $\Omega$ is a Cartesian product of copies of $B^{n}$.

Proof. (a) The zeros of holomorphic bisectional curvature are preserved by a holomorphic isometry. Thus, whenever $R_{\alpha \bar{\alpha} \zeta \bar{\zeta}}^{D}=0$, we have $R_{\alpha \bar{\alpha} \zeta \bar{\zeta}}^{\Omega}=0$ and $\left\|\sigma_{\alpha \zeta}\right\|^{2}=R_{\alpha \bar{\alpha} \zeta \bar{\zeta}}^{\Omega}-$ $R_{\alpha \bar{\alpha} \zeta \bar{\zeta}}^{D}=0$. When $D$ is irreducible and of rank $\geq 2$ the partial vanishing $\sigma_{\alpha \zeta}=0$ is enough to imply $\sigma \equiv 0$, by Mok [Mk1, proof of Corollary to Theorem 3', p. $138 \mathrm{ff}$.] (cf. also Clozel-Ullmo [CU, §3]). Assume now that $D$ is reducible, $D=D_{1} \times \cdots \times D_{k}$, $k \geq 2$, and each irreducible component $D_{i}, 1 \leq i \leq k$, is of rank $\geq 2$. Fix $x \in D$. For $\eta_{i}, \eta_{i}^{\prime} \in T_{x}(D)$ tangent to the $i$-th direct factor we have $\sigma_{\eta_{i}} \eta_{i}^{\prime}=0$. On the other hand, if $\eta_{j} \in T_{x}(D)$ is tangent to the $j$-th direct factor and $i \neq j$, then $R_{\eta_{i} \overline{\eta_{i}}}^{D} \eta_{j} \overline{\eta_{j}}=0$, and we deduce by $\left\|\sigma_{\eta_{i} \eta_{j}}\right\|^{2}=R_{\eta_{i} \overline{\eta_{i}} \eta_{j} \overline{\eta_{j}}}-R_{\eta_{i} \overline{\eta_{i}} \eta_{j} \overline{\eta_{j}}}^{D}=0$ that $\sigma_{\eta_{i} \eta_{j}}=0$. From $\sigma_{\eta_{i} \eta_{i}^{\prime}}=\sigma_{\eta_{i} \eta_{j}}=0$ we conclude that $\sigma \equiv 0$ on $D$, proving that $f:\left(D, \lambda d s_{D}^{2} ; 0\right) \rightarrow\left(\Omega, d s_{\Omega}^{2} ; 0\right)$ is totally geodesic.

(b) The statement for the germ of map $f:\left(B^{n}, \lambda d s_{B^{n}}^{2} ; 0\right) \rightarrow\left(\left(B^{n}\right)^{p}, d s_{\left(B^{n}\right)}^{2} ; 0\right)$ was established in Mok [Mk3] under the assumptions that (i) the normalizing constant $\lambda$ is a positive integer, and that (ii) writing $f=\left(f_{1}, \ldots, f_{p}\right), f_{i}: B^{n} \rightarrow B^{n}$, for $1 \leq i \leq p$, each $f_{i}$ is of maximal rank at some point. When the normalizing constant $\lambda>0$ is an arbitrary positive real number, results of the current article apply. In fact, by Theorem 1.3.1, Graph $(f)$ extends as an affine-algebraic variety. The final argument in [Mk3] using Alexander's Theorem remains valid to show that $f$ is totally geodesic, as follows. The functional identities as in Proposition 1.1.1 apply and we have in particular the identity

$$
\prod_{i=1}^{p}\left(1-\left\|f_{i}\right\|^{2}\right)=\left(1-\|z\|^{2}\right)^{\lambda}
$$

analogous to Mok [Mk3, proof of Theorem (3.1)]. Pick $b \in \partial B^{n}$ where $f$ extends holomorphically to a neighborhood $U_{b}$ of $b$ in $\mathbb{C}^{n}$. From (1) one of the factors $1-\left\|f_{i}\right\|^{2}$, $1 \leq i \leq p$, must vanish on $\partial B^{n}$. We may take $i=p$. Since $n \geq 2$ and $f_{p}$ is obviously nonconstant, Alexander's Theorem (stated below) applies to force $f_{p}$ to extend to a biholomorphism $F_{p}: B^{n} \rightarrow B^{n}$. Since $f_{p}(0)=0$ we must have $\left\|f_{p}(z)\right\|=\|z\|$, hence by (1) we have $\prod_{i=1}^{p-1}\left(1-\left\|f_{i}\right\|^{2}\right)=\left(1-\|z\|^{2}\right)^{\lambda-1}$, and (b) follows by induction, as desired.

Theorem (Alexander [Al]). Let $B^{n} \Subset \mathbb{C}^{n}$ be the complex unit ball of dimension $n \geq 2$. Let $b \in \partial B^{n}, U_{b}$ be a connected open neighborhood of $b$ in $\mathbb{C}^{n}$, and $f: U_{b} \rightarrow \mathbb{C}^{n}$ be a non-constant holomorphic map such that $f\left(U_{b} \cap \partial B^{n}\right) \subset \partial B^{n}$. Then there exists an automorphism $F: B^{n} \rightarrow B^{n}$ such that $\left.\left.F\right|_{U_{b} \cap B^{n}} \equiv f\right|_{U_{b} \cap B^{n}}$. 


\section{Generalizations of extension results for bounded domains and for complex manifolds}

\subsection{Extension of germs of holomorphic isometries for bounded domains}

We have considered the extension problem for bounded complete circular domains on germs of holomorphic isometries $f$ at 0 , where $f(0)=0$. Here we generalize the results to holomorphic isometries $f:\left(D ; \lambda d s_{D}^{2} ; x_{0}\right) \rightarrow\left(\Omega, d s_{\Omega}^{2} ; f\left(x_{0}\right)\right)$ between arbitrary bounded domains.

Theorem 2.1.1. Let $D \Subset \mathbb{C}^{n}$ and $\Omega \Subset \mathbb{C}^{N}$ be bounded domains. Let $x_{0} \in D, \lambda$ be a positive real number, and $f:\left(D, \lambda d s_{D}^{2} ; x_{0}\right) \rightarrow\left(\Omega, d s_{\Omega}^{2} ; f\left(x_{0}\right)\right)$ be a germ of holomorphic isometry. Then the germ of complex-analytic subvariety $\operatorname{Graph}(f)$ at $\left(x_{0}, f\left(x_{0}\right)\right)$ extends to an irreducible complex-analytic subvariety $S \subset D \times \Omega$ which is the graph of a holomorphic isometric embedding $F:\left(D^{\prime},\left.\lambda d s_{D}^{2}\right|_{D^{\prime}}\right) \rightarrow\left(\Omega, d s_{\Omega}^{2}\right)$ defined on some neighborhood $D^{\prime}$ of $x_{0}$ in $D$. If $\left(\Omega, d s_{\Omega}^{2}\right)$ is complete as a Kähler manifold, then $D^{\prime}=D$, so that the germ of holomorphic isometric immersion $f$ extends to a holomorphic isometric embedding $F:\left(D, \lambda d s_{D}^{2}\right) \rightarrow\left(\Omega, d s_{\Omega}^{2}\right)$.

In what follows for $\epsilon, \delta>0$ sufficiently small we will write $D_{\epsilon}:=B^{n}\left(x_{0} ; \epsilon\right) \Subset D$ and $\Omega_{\delta}:=B^{N}\left(x_{0} ; \delta\right) \Subset \Omega$. The germ of holomorphic map $f:\left(D ; x_{0}\right) \rightarrow\left(\Omega ; f\left(x_{0}\right)\right)$ will be taken to be defined on some $D_{\epsilon_{0}}, \epsilon_{0}>0$ being sufficiently small and fixed.

Proof of Theorem 2.1.1. With some minor differences Theorem 1.2.1 deals with the special case where $D \Subset \mathbb{C}^{n}$ and $\Omega \Subset \mathbb{C}^{N}$ are complete circular domains, $x_{0}=0$, and $f\left(x_{0}\right)=0$. In the proof there we made use of the circle group action. With reference to the proof given there and in the same notation, we examine what is needed on the coordinates $\left(z_{i}\right)$ and $\left(\zeta_{j}\right)$ for the proof to work. We have

$$
\log K_{\Omega}(f(z), f(z))=\lambda \log K_{D}(z, z)+\operatorname{Re}(\psi),
$$

where $\psi$ is a holomorphic function on $D_{\epsilon_{0}}$. The pluriharmonic function $\operatorname{Re}(\psi)$ is shown to be a constant by the observations that for $|I| \neq|J|$, (a) the coefficient of $z^{I} \overline{z^{J}}$ in $\log K_{D}(z, z)$ is always 0 ; and (b) the coefficient of $\zeta^{I} \zeta^{J}$ in $\log K_{\Omega}(\zeta, \zeta)$ is always 0 . By (b), substituting $\zeta=f(z)$ satisfying $f(0)=0$, we conclude that the coefficient of $z^{I}$ (and hence of $\left.\overline{z^{I}}\right)$ in $\log K_{\Omega}(f(z), f(z))$ is always 0 whenever $I=\left(i_{1}, \ldots, i_{n}\right)$ is non-zero. Using (a) and (b) and comparing the two sides of (1) it follows that $\psi$ must be a constant.

The observations (a) and (b) hold true because of the invariance of the Bergman kernels under the circle group action at 0 . But, in order to conclude that $\psi$ is a constant, it is sufficient that whenever $I=\left(i_{1}, \ldots, i_{n}\right)$ is non-zero, $\left(\mathrm{a}^{\prime}\right)$ the coefficient of $\left(z-x_{0}\right)^{I}$ in $\log K_{D}(z, z)$ is always 0 ; and $\left(\mathrm{b}^{\prime}\right)$ the coefficient of $\left(\zeta-f\left(x_{0}\right)\right)^{I}$ in $\log K_{\Omega}(\zeta, \zeta)$ is always 0 . Such coordinates do not always exist. However, in place of using $\log K_{D}(z, z)$, resp. $\log K_{\Omega}(\zeta, \zeta)$, we can first remove pluriharmonic functions from the potential functions before comparing the two sides in the functional equations. For $\left(\mathrm{a}^{\prime}\right)$ and $\left(\mathrm{b}^{\prime}\right)$ to hold true it suffices that we choose a potential function at $x_{0}$ for the Bergman metric which is a convergent sum of $\pm|\theta|^{2}$ for a countable number of holomorphic functions $\theta$ 
on $D$ vanishing at $x_{0}$, and an analogous potential function at $y_{0}:=f\left(x_{0}\right)$. For this purpose let $\left(s_{0}, s_{1}, \ldots\right)$ be an orthonormal basis of $H^{2}(D)$ adapted to $x_{0}$ so that $s_{i}\left(x_{0}\right)=0$ for $i \geq 1$. Then the Bergman kernel $K_{D}$ is given by $K_{D}(z, z)=\left|s_{0}\right|^{2} K_{D}^{\prime}(z, z)$, where $K_{D}^{\prime}(z, z)=1+\sum_{i>1}\left|s_{i} / s_{0}\right|^{2}$. Expanding in power series on some neighborhood of $x_{0}$, the function $\log K_{D}^{\prime}(z, z)$ is the convergent sum of a countable number of functions of the form $\pm\left|\theta_{k}\right|^{2}$, where each $\theta_{k}$ is a holomorphic function vanishing at $x_{0}$. Choose now analogously an orthonormal basis $\left(r_{0}, r_{1}, \ldots\right)$ of $H^{2}(\Omega)$ adapted to $y_{0}$ so that $r_{j}\left(y_{0}\right)=0$ for every $j \geq 1$, and write in a similar way $K_{\Omega}(\zeta, \zeta)=\left|r_{0}\right|^{2} K_{\Omega}^{\prime}(\zeta, \zeta)$. Again, on some neighborhood of $y_{0}$ the function $\log K_{\Omega}^{\prime}(\zeta, \zeta)$ is the convergent sum of a countable number of functions of the form $\pm\left|\chi_{\ell}\right|^{2}$, where each $\chi_{\ell}$ is a holomorphic function on $\Omega$ vanishing at $y_{0}$. Noting that $\log \left|s_{0}\right|^{2}$, resp. $\log \left|r_{0}\right|^{2}$, is a pluriharmonic function on a neighborhood of $x_{0}$, resp. $y_{0}$, the hypothesis that $f:\left(D, x_{0}\right) \rightarrow\left(\Omega, y_{0}\right)$ is a holomorphic isometry up to a normalizing constant gives rise to

$$
\begin{aligned}
\sqrt{-1} \partial \bar{\partial} \log K_{\Omega}(f(z), f(z)) & =\lambda \sqrt{-1} \partial \bar{\partial} \log K_{D}(z, z), \\
\log K_{\Omega}^{\prime}(f(z), f(z)) & =\lambda \log K_{D}^{\prime}(z, z)+\operatorname{Re}\left(\psi^{\prime}\right),
\end{aligned}
$$

where $\psi^{\prime}$ is a germ of holomorphic function at $x_{0}$. Thus, we have $\log K_{\Omega}^{\prime}(f(z), f(z))=$ $\sum_{\ell} \pm\left|\left(\chi_{\ell} \circ f\right)(z)\right|^{2}$, where $\left(\chi_{\ell} \circ f\right)\left(x_{0}\right)=\chi_{\ell}\left(f\left(x_{0}\right)\right)=\chi_{\ell}\left(y_{0}\right)=0$. Expanding in power series at $x_{0}$ and observing that $2 \operatorname{Re}\left(\psi^{\prime}\right)=\psi^{\prime}+\overline{\psi^{\prime}}$ is a sum of terms of pure type, it follows that in fact the pluriharmonic function $\operatorname{Re}\left(\psi^{\prime}\right)$ vanishes identically, giving

$$
\log K_{\Omega}^{\prime}(f(z), f(z))=\lambda \log K_{D}^{\prime}(z, z) .
$$

From $K_{D}^{\prime}(z, z)$ we define the function $K_{D}^{\prime}(z, w)$ holomorphic in $z$ and anti-holomorphic in $w$ such that one recovers the original definition by restricting to $z=w$. The same applies to $K_{\Omega}^{\prime}(\zeta, \xi)$. Writing the extremal functions $s_{0} \in H^{2}(D)$ and $r_{0} \in H^{2}(\Omega)$ as

$$
s_{0}(z)=\frac{K_{D}\left(z, x_{0}\right)}{\sqrt{K_{D}\left(x_{0}, x_{0}\right)}}, \quad r_{0}(\zeta)=\frac{K_{\Omega}\left(\zeta, y_{0}\right)}{\sqrt{K_{\Omega}\left(y_{0}, y_{0}\right)}},
$$

from $K_{D}^{\prime}(z, w)=\frac{K_{D}(z, w)}{s_{0}(z) \overline{s_{0}(w)}}$ and $K_{\Omega}^{\prime}(\zeta, \xi)=\frac{K_{\Omega}(\zeta, \xi)}{r_{0}(\zeta) \overline{r_{0}(\xi)}}$ we have

$$
K_{D}^{\prime}(z, w)=\frac{K_{D}(z, w) K_{D}\left(x_{0}, x_{0}\right)}{K_{D}\left(z, x_{0}\right) K_{D}\left(x_{0}, w\right)}, \quad K_{\Omega}^{\prime}(\zeta, \xi)=\frac{K_{\Omega}(\zeta, \xi) K_{\Omega}\left(y_{0}, y_{0}\right)}{K_{\Omega}\left(\zeta, y_{0}\right) K_{\Omega}\left(y_{0}, \xi\right)}
$$

Observe from (4) that

$$
K_{D}^{\prime}\left(z, x_{0}\right)=1, \quad K_{\Omega}^{\prime}\left(\zeta, y_{0}\right)=1 .
$$

Let $\left(h_{j}\right)_{j=0}^{\infty}$ be an orthonormal basis of $H^{2}(\Omega)$ and write $h_{j}^{\prime}=h_{j} / r_{0}$. Define $\Phi: \Omega \rightarrow \mathbb{H}$ by

$$
\Phi(\zeta)=\left(h_{0}(\zeta), h_{1}(\zeta), \ldots\right)
$$

We also write

$$
\Phi^{\prime}(\zeta)=\left(h_{0}^{\prime}(\zeta), h_{1}^{\prime}(\zeta), \ldots\right)=\frac{\Phi(\zeta)}{r_{0}(\zeta)}
$$


Each component $h_{j}^{\prime}$ of $\Phi^{\prime}$ is meromorphic on $\Omega$ and may in general have poles. However, since $r_{0}\left(y_{0}\right) \neq 0$, without loss of generality we will assume that $f\left(D_{\epsilon_{0}}\right) \subset \Omega_{\delta_{0}}$ where $r_{0}$ has no zeros on $\Omega_{\delta_{0}}$, so that $\Phi^{\prime} \circ f$ is holomorphic on $D_{\epsilon_{0}}$. We are going to prove the extendibility of $\operatorname{Graph}(f)$ to $S \subset D \times \Omega$ as a complex-analytic subvariety by imposing first of all the following simplifying assumption on the Bergman kernel $K_{D}(z, w)$ :

$(\sharp)$ The holomorphic function $K_{D}\left(z, x_{0}\right)$ of $z$ does not have any zero on $D$.

Assuming $(\sharp)$, the function $K_{D}^{\prime}(z, w)$ is holomorphic in $(z, \bar{w})$ on $D \times D$. Let $G \Subset D$ be an open neighborhood of $\overline{D_{\epsilon_{0}}}$. Since $K_{D}^{\prime}\left(z, x_{0}\right) \equiv 1$ by (5), from the continuity of $K_{D}^{\prime}(z, w)$, for some $\epsilon$ satisfying $0<\epsilon \leq \epsilon_{0}$ we must have $\operatorname{Re}\left(K_{D}^{\prime}(z, w)\right)>0$ whenever $(z, w) \in G \times D_{\epsilon}$. Thus, for $(z, w) \in G \times D_{\epsilon}$, the function $\log K_{D}^{\prime}(z, w)$ is well-defined and holomorphic in $(z, \bar{w})$ for the principal branch log of the natural logarithm, so that $\left(K_{D}^{\prime}(z, w)\right)^{\lambda}=\exp \left(\lambda \log K_{D}^{\prime}(z, w)\right)$ is defined and holomorphic in $(z, \bar{w})$ there. Consider

$$
\left(\mathbf{I}_{w}\right) \quad K_{\Omega}^{\prime}(\zeta, f(w))=\left(K_{D}^{\prime}(z, w)\right)^{\lambda}, \quad w \in D_{\epsilon},
$$

restricted to $(z, \zeta) \in G \times \Omega$, and denote by $V_{G} \subset G \times \Omega$ the set of common solutions to $\left(\mathbf{I}_{w}\right), w \in D_{\epsilon}$. By polarizing (3) and exponentiating, it follows that $\left(\mathbf{I}_{w}\right)$ is satisfied by $\zeta=f(z)$ for $w \in D_{\epsilon}$. Suppose connected open subsets $G$ and $G^{\prime}$ are chosen such that $D_{\epsilon} \Subset G \Subset G^{\prime} \Subset D$ and $\epsilon, \epsilon^{\prime}$ are chosen so that $0<\epsilon^{\prime}<\epsilon \leq \epsilon_{0}$ and $\operatorname{Re}\left(K_{D}^{\prime}(z, w)\right)>0$ whenever $(z, w) \in G \times D_{\epsilon}$ or $(z, w) \in G^{\prime} \times D_{\epsilon^{\prime}}$. Then $V_{G^{\prime}} \cap(G \times \Omega)=V_{G}$ by the Identity Theorem for holomorphic functions. Choose a sequence $\left(G_{k}\right)_{k=1}^{\infty}$ of connected open subsets of $D$ such that $D_{\epsilon} \Subset \cdots \Subset G_{k} \Subset G_{k+1} \Subset \cdots \Subset D$ and $\bigcup_{k \geq 1} G_{k}=D$, and a corresponding strictly decreasing sequence of positive numbers $\left(\epsilon_{k}\right)_{k=1}^{\infty}$ converging to 0 such that $\operatorname{Re}\left(K_{D}^{\prime}(z, w)\right)>0$ whenever $(z, w) \in G_{k} \times D_{\epsilon_{k}}$ for some integer $k \geq 1$. Then the union $V=\bigcup_{k \geq 1} V_{G_{k}}$ is a subvariety of $D \times \Omega$.

Let $U \subset D_{\epsilon_{0}}$, and let $\left\{f_{t}(z)\right\}$ for $t \in \Delta$ and $z \in U$ be a holomorphic 1-parameter family of solutions to the functional equations $\left(\mathbf{I}_{w}\right), w \in D_{\epsilon}$, given by

$$
K_{\Omega}^{\prime}\left(f_{t}(z), f(w)\right)=\left(K_{D}^{\prime}(z, w)\right)^{\lambda}, \quad w \in D_{\epsilon},
$$

as $w$ ranges over $D_{\epsilon}$. Write $\Sigma:=f(U) \subset \Omega$ and $\Xi^{\prime}:=\Phi^{\prime}(\Sigma) \subset \mathbb{H}$. Again, let $\ell$ be the first positive integer such that $\left.\frac{\partial^{\ell}}{\partial t^{\ell}} f_{t}(z)\right|_{t=0}$ is not identically zero on $U$. Then, as in the proof of Proposition 1.1.2, differentiating the identities (6) against $t$ exactly $\ell$ times and evaluating at $t=0$ we obtain a holomorphic vector field $\eta(f(z))$ on $\Sigma$ and a corresponding holomorphic vector field along $d \Phi^{\prime}(\eta)$ along $\Xi^{\prime}$ satisfying

$$
\left\langle d \Phi^{\prime}(\eta(f(z))), \overline{\Phi^{\prime}(f(w))}\right\rangle=0 .
$$

Write

$$
\begin{aligned}
K_{\Omega}^{\prime}(\zeta, \xi) & =\frac{1}{r_{0}(\zeta) \overline{r_{0}(\xi)}} K_{\Omega}(\zeta, \xi)=\frac{1}{r_{0}(\zeta) \overline{r_{0}(\xi)}}\left(h_{0}(\zeta) \overline{h_{0}(\xi)}+h_{1}(\zeta) \overline{h_{1}(\xi)}+\cdots\right) \\
& =h_{0}^{\prime}(\zeta) \overline{h_{0}^{\prime}(\xi)}+h_{1}^{\prime}(\zeta) \overline{h_{1}^{\prime}(\xi)}+\cdots
\end{aligned}
$$


Choose now the orthonormal basis $\left(h_{0}, h_{1}, \ldots\right)$ of $H^{2}(\Omega)$ adapted to a point $z_{0}$ on $U$ and $\eta\left(f\left(z_{0}\right)\right)$ as in the proof of Proposition 1.1.2, so that $h_{j}\left(z_{0}\right)=0$ whenever $j \geq 1$, and $d h_{j}\left(\eta\left(f\left(z_{0}\right)\right)\right)=0$ whenever $j \geq 2$. Clearly, we also have $h_{j}^{\prime}\left(z_{0}\right)=0$ whenever $j \geq 1$, and $d h_{j}^{\prime}\left(\left(\eta\left(f\left(z_{0}\right)\right)\right)=0\right.$ whenever $j \geq 2$. By the analogues of (2)-(4) in the proof of Lemma 1.1.2, applied instead to $\left(h_{j}^{\prime}\right)_{j=0}^{\infty}$, we conclude that $h_{1}^{\prime}(f(w))=0$ and hence $h_{1}(f(w))=0$ for any $w \in D_{\epsilon}$. Defining $\mathcal{E} \subset H^{2}(\Omega)$ to consist of $h_{1}=h_{\eta, z_{0}}$ from infinitesimal variations of solutions to $\left(\mathbf{I}_{w}\right)$, and $E \subset \Omega$ to consist of common zeros of $h_{\alpha} \in \mathcal{E}$ (cf. the proof of Proposition 1.1.2), the irreducible component $S$ of $V \cap(D \times E)$ containing Graph $(f)$ gives an extension of $\operatorname{Graph}(f)$ to a subvariety of $D \times \Omega$.

In the absence of $(\sharp)$ there is the problem of making sense of the identity (3) and its polarization, formally written $\log K_{\Omega}^{\prime}(f(z), f(w))=\lambda \log K_{D}^{\prime}(z, w)$, both sides of which can only be understood as multi-valued functions when the domain of definition of $f: D_{\epsilon_{0}} \rightarrow \Omega$ is enlarged. Recall that for $z, w \in D$ we write $K_{D, w}(z)=K_{D}(z, w)$ and likewise for $(\zeta, \xi) \in \Omega$ we write $K_{\Omega, \xi}(\zeta)=K_{\Omega}(\zeta, \xi)$. For each $w \in D_{\epsilon_{0}}$, denote by $\Theta_{w} \Subset D \times \Omega$ the complex-analytic subvariety given by

$$
\Theta_{w}:=\left(\left(\operatorname{Zero}\left(K_{D, x_{0}}\right) \cup \operatorname{Zero}\left(K_{D, w}\right)\right) \times \Omega\right) \cup\left(D \times\left(\operatorname{Zero}\left(K_{\Omega, f\left(x_{0}\right)}\right) \cup \operatorname{Zero}\left(K_{\Omega, f(w)}\right)\right)\right) .
$$

Given a relatively compact subdomain in $D \times \Omega-\Theta_{x_{0}}$ we will consider functional equations $\left(\mathbf{J}_{w}\right)$ which are well-defined on the subdomain provided that $w$ is sufficiently close to $x_{0}$, where the requirement of proximity of $w$ to $x_{0}$ depends on the subdomain chosen.

Let $G \Subset D-\operatorname{Zero}\left(K_{D, x_{0}}\right)$ and $\mathcal{O} \Subset \Omega-\operatorname{Zero}\left(K_{\Omega, f\left(x_{0}\right)}\right)$ be arbitrary relatively compact subdomains. Observe that for $\epsilon>0$ sufficiently small, $K_{D}^{\prime}(z, w)$ is holomorphic in $(z, \bar{w})$ for $(z, w) \in G \times D_{\epsilon}$, and we have $K_{D}^{\prime}\left(z, x_{0}\right) \equiv 1$ for $z \in D-\operatorname{Zero}\left(K_{D, x_{0}}\right)$. Likewise for $\delta>0$ sufficiently small, $K_{\Omega}^{\prime}(\zeta, \xi)$ is holomorphic in $(\zeta, \bar{\xi})$ for $(\zeta, \xi) \in$ $\mathcal{O} \times \Omega_{\delta}$, and we have $K_{\Omega}^{\prime}\left(\zeta, f\left(x_{0}\right)\right) \equiv 1$ for $\zeta \in \Omega-\operatorname{Zero}\left(K_{\Omega, f\left(x_{0}\right)}\right)$. Hence, for some $\epsilon=\epsilon(G, \mathcal{O})<\epsilon_{0}$ we have $\operatorname{Re}\left(K_{D}^{\prime}(z, w)\right)>0$ and $\operatorname{Re}\left(K_{\Omega}^{\prime}(\zeta, f(w))\right)>0$ whenever $w \in D_{\epsilon}$ and $(z, \zeta) \in G \times \mathcal{O}$. Let $W_{G}$ be the set of common solutions $(z, \zeta) \in G \times \mathcal{O}$ to the functional equations

$$
\left(\mathbf{I}_{w}^{\prime}\right) \quad \log K_{\Omega}^{\prime}(\zeta, f(w))=\lambda \log K_{D}^{\prime}(z, w), \quad w \in D_{\epsilon},
$$

where log stands for the principal branch of logarithm. $W_{G}$ contains $\operatorname{Graph}(f)$ and the germs of $W_{G}$ and $V_{G}$ at a general point of Graph $(f)$ agree with each other. Using (4) we have the following equivalent family of functional equations:

$$
\begin{aligned}
\left(\mathbf{J}_{w}\right) \quad H(z, \zeta ; w):= & \log \left(\frac{K_{\Omega}(\zeta, f(w))}{K_{\Omega}\left(\zeta, f\left(x_{0}\right)\right) K_{\Omega}\left(f\left(x_{0}\right), f(w)\right)}\right) \\
& -\lambda \log \left(\frac{K_{D}(z, w)}{K_{D}\left(z, x_{0}\right) K_{D}\left(x_{0}, w\right)}\right)+a=0, \quad w \in D_{\epsilon},
\end{aligned}
$$

where $a=\log K_{\Omega}\left(f\left(x_{0}\right), f\left(x_{0}\right)\right)-\lambda \log K_{D}\left(x_{0}, x_{0}\right)$. Thus $H_{w}(z, \zeta):=H(z, \zeta ; w)$ is a holomorphic function on $G \times \mathcal{O}$. Note that $H(z, \zeta ; w)$ depends anti-holomorphically on 
$w \in D_{\epsilon}$. For $1 \leq i \leq n$ and $w \in D_{\epsilon}$ consider now the new equations $\left(\mathbf{L}_{w}^{i}\right)$ defined by differentiating the equations $\left(\mathbf{J}_{w}\right)$, given by

$$
\left(\mathbf{L}_{w}^{i}\right) \quad L_{w}^{i}(z, \zeta):=\frac{\partial H_{w}}{\partial \overline{w_{i}}}(z, \zeta)=0
$$

where by definition $\frac{\partial H_{w}}{\partial \bar{w}_{i}}(z, \zeta)=\frac{\partial}{\partial \bar{w}_{i}} H(z, \zeta ; w)$. More explicitly we have

$$
\begin{aligned}
\left(\mathbf{L}_{w}^{i}\right) \quad \frac{\left.\sum_{j=1}^{N} \frac{\partial}{\partial \bar{\xi}_{j}} K_{\Omega}(\zeta, \xi)\right|_{\xi=f(w)} \frac{\partial \overline{f j}}{\partial \overline{w_{i}}}(w)}{K_{\Omega}(\zeta, f(w))} & -\frac{\left.\sum_{j=1}^{N} \frac{\partial}{\partial \bar{\xi}_{j}} K_{\Omega}\left(f\left(x_{0}\right), \xi\right)\right|_{\xi=f(w)} \frac{\partial \overline{f^{j}}}{\partial \overline{w_{i}}}(w)}{K_{\Omega}\left(f\left(x_{0}\right), f(w)\right)} \\
& -\lambda\left(\frac{\frac{\partial}{\partial \bar{w}_{i}} K_{D}(z, w)}{K_{D}(z, w)}-\frac{\frac{\partial}{\partial \bar{w}_{i}} K_{D}\left(x_{0}, w\right)}{K_{D}\left(x_{0}, w\right)}\right)=0
\end{aligned}
$$

which shows that each $L_{w}^{i}(z, \zeta)$, a priori only defined on $G \times \mathcal{O}$, extends meromorphically to $D \times \Omega$, a crucial fact in what follows. To proceed we need the following obvious lemma.

Lemma 2.1.1. Let $U \subset \mathbb{C}^{m}$ be a domain and $E \subset U$ be the common zero set of a realanalytic family $\left\{\varphi_{t}: t=\left(t_{1}, \ldots, t_{s}\right) \in(-1,1)^{s}\right\}$ of holomorphic functions parametrized by an open cube $(-1,1)^{s} \subset \mathbb{R}^{s}$. Write $\psi(z, t):=\varphi_{t}(z)$, and define $\psi_{t, i}(z):=\frac{\partial \psi}{\partial t_{i}}(z, t)$. Then $E$ is the common zero set of $\varphi_{0}$ and of $\left\{\psi_{t, i}: t \in(-1,1)^{s}, 1 \leq i \leq s\right\}$.

Returning to Theorem 2.1.1, for $w \in D_{\epsilon}$ consider the real-analytic family of holomorphic functions $H_{w}(z, \zeta):=H(z, \zeta ; w)$ on $D \times \Omega$ as being parametrized by the real $2 n$ dimensional parameter space $D_{\epsilon}$ in the variables $\left(\operatorname{Re}\left(w_{i}\right), \operatorname{Im}\left(w_{i}\right)\right), 1 \leq i \leq n$. Observe the crucial fact that $H_{x_{0}}(z, \zeta)=0$ when $w=x_{0}$, so that in the application of Lemma 2.1.1 the function $\varphi_{0}$ there is the zero function, leaving us with only the first derivatives of $H_{w}(z, \zeta)$ against $w$. Since $H_{w}$ varies anti-holomorphically in $w$, to apply Lemma 2.1.1 above it suffices to take the first derivatives against $\overline{w_{i}}, 1 \leq i \leq n$, i.e., to consider $L_{w}^{i}(z, \zeta)=\frac{\partial H_{w}}{\partial \bar{w}_{i}}(z, \zeta)$. Recall that for $(z, \zeta) \in G \times \mathcal{O}$ the functional equation $\left(\mathbf{J}_{w}\right)$ for $w \in D_{\epsilon}$ is well-defined. More generally, let $\left(G_{k}\right)_{k=1}^{\infty}$ be a sequence of subdomains of $D-\operatorname{Zero}\left(K_{D, x_{0}}\right)$ such that $G_{1} \Subset G_{2} \Subset \cdots \Subset D$ and $\bigcup_{k>1} G_{k}=D-\operatorname{Zero}\left(K_{D, x_{0}}\right)$, and likewise let $\left(\mathcal{O}_{k}\right)_{k=1}^{\infty}$ be a sequence of subdomains of $\Omega-\operatorname{Zero}\left(K_{\Omega, f\left(x_{0}\right)}\right)$ such that $\mathcal{O}_{1} \Subset$ $\mathcal{O}_{2} \Subset \cdots \Subset \Omega$ and $\bigcup_{k \geq 1} \mathcal{O}_{k}=\Omega-\operatorname{Zero}\left(K_{\Omega, f\left(x_{0}\right)}\right)$. Then there exists a strictly decreasing sequence $\left(\epsilon_{k}\right)_{k=1}^{\infty}$ of positive numbers converging to 0 such that $\operatorname{Re}\left(K_{D}^{\prime}(z, w)\right)>0$ whenever $(z, w) \in G_{k} \times D_{\epsilon_{k}}$ for some $k \geq 1$, and $\operatorname{Re}\left(K_{\Omega}^{\prime}(\zeta, f(w))\right)>0$ whenever $(w, \zeta) \in D_{\epsilon_{k}} \times \mathcal{O}_{k}$ for some $k \geq 1$. Thus, given $z \in G_{k}, H_{w}(z, \zeta)$ is defined whenever $(w, \zeta) \in D_{\epsilon_{k}} \times \mathcal{O}_{k}$. Define now the subvariety $W \subset(D \times \Omega)-\Theta_{x_{0}}$, resp. $V^{\prime} \subset D \times \Omega$, by $\left(\mathbf{J}_{w}\right)$, resp. $\left(\mathbf{L}_{w}^{i}\right)$, as follows:

$$
\begin{aligned}
& W:=\left\{(z, \zeta) \in(D \times \Omega)-\Theta_{x_{0}}: H_{w}(z, \zeta)=0 \text { for all } w \text { sufficiently close to } x_{0}\right\} \\
& V^{\prime}:=\left\{(z, \zeta) \in D \times \Omega: L_{w}^{i}(z, \zeta)=0 \text { for all } w \in D_{\epsilon}, 1 \leq i \leq n\right\} .
\end{aligned}
$$

Thus, $V^{\prime}$ is the common solution set of $\left(\mathbf{L}_{w}^{i}\right), w \in D_{\epsilon}, 1 \leq i \leq n$, i.e., the intersection of the zero sets of the meromorphic functions $L_{w}^{i}(z, \zeta)$ on $D \times \Omega$. By Lemma 2.1.1, 
$W$ agrees with $V^{\prime} \cap\left((D \times \Omega)-\Theta_{x_{0}}\right)$, hence $\operatorname{Graph}(f) \subset V^{\prime}$. In terms of exhaustion sequences as explained above, for $k \geq 1$ and for $(z, \zeta) \in G_{k} \times \mathcal{O}_{k}$ we consider only the functional equations for $H_{w}(z, \zeta)$ for $w \in D_{\epsilon_{k}}$. If we denote by $W_{k} \subset G_{k} \times \mathcal{O}_{k}$ the intersection of the zero sets of $H_{w}(z, \zeta)$ as $w$ ranges over $D_{\epsilon_{k}}$, then $W_{k+1} \cap\left(G_{k} \times\right.$ $\left.\mathcal{O}_{k}\right)=W_{k}$ for $k \geq 1$ by the Identity Theorem for (anti-)holomorphic functions, and we have $W=\bigcup_{k \geq 1} W_{k}$.

Using $V^{\prime}$ in place of $V$ (as in the case with the additional assumption $(\sharp)$ ) and the same extremal functions $h_{\alpha} \in \mathcal{E}$, with common zero set $E \subset \Omega$, the irreducible component $S$ of $T:=V^{\prime} \cap(D \times E)$ containing $\operatorname{Graph}(f)$ gives the desired analytic continuation of Graph $(f)$ to a subvariety of $D \times \Omega$. To prove that $S$ is the graph of some holomorphic isometry $F:\left(D^{\prime},\left.\lambda d s_{D}^{2}\right|_{D^{\prime}}\right) \rightarrow\left(\Omega, d s_{\Omega}^{2}\right)$, by the arguments of Theorem 1.2.1 and using the identities (3) above, for two branches $f_{1}(z), f_{2}(z)$ of the analytic continuation of $f$ over some subdomain of $D$, we have

$$
K_{\Omega}^{\prime}\left(f_{1}(z), f_{1}(z)\right)=K_{\Omega}^{\prime}\left(f_{1}(z), f_{2}(z)\right)=K_{\Omega}^{\prime}\left(f_{2}(z), f_{2}(z)\right)
$$

Since $K_{\Omega}(\zeta, \xi)=\frac{\left.K_{\Omega}\left(\zeta, y_{0}\right) K_{\Omega}\left(y_{0}, \xi\right)\right)}{K_{\Omega}\left(y_{0}, y_{0}\right)} K_{\Omega}^{\prime}(\zeta, \xi)$, we conclude from (7) that

$$
\left|K_{\Omega}\left(f_{1}(z), f_{2}(z)\right)\right|^{2}=K_{\Omega}\left(f_{1}(z), f_{1}(z)\right) K_{\Omega}\left(f_{2}(z), f_{2}(z)\right)
$$

Write $\Phi: \Omega \rightarrow \mathbb{H}$ for the canonical map defined in terms of any orthonormal basis of $H^{2}(\Omega)$. By the Cauchy-Schwarz inequality, it follows from (8) that for some non-zero complex number $c$ we have

$$
\Phi\left(f_{1}(z)\right)=c \Phi\left(f_{2}(z)\right), \quad \text { so that } \quad f_{1}(z)=f_{2}(z)
$$

Consequently, the $\operatorname{argument} S=\operatorname{Graph}(F)$ for some $F:\left(D^{\prime},\left.\lambda d s_{D}^{2}\right|_{D^{\prime}}\right) \rightarrow\left(\Omega, d s_{\Omega}^{2}\right)$ works verbatim as in the proof of Theorem 1.2.1. The proof of injectivity of $F$ is also the same. Finally, supposing that $\left(\Omega, d s_{\Omega}^{2}\right)$ is complete as a Kähler manifold, we have to prove that $D^{\prime}=D$. Suppose otherwise, i.e., $D^{\prime} \subsetneq D$. Let $r \in \partial D^{\prime} \cap D$ and $\gamma:[0,1] \rightarrow D$ be a smooth curve such that $\gamma(0)=x_{0}$ and $\gamma(1)=r$. Among $t \in[0,1]$ let $t_{0}$ be the first element such that $\gamma\left(t_{0}\right) \notin D^{\prime}$ and write $p=\gamma\left(t_{0}\right)$. Since $F:\left(D^{\prime}, \lambda d s_{D}^{2}\right) \rightarrow\left(\Omega, d s_{\Omega}^{2}\right)$ is a holomorphic isometry, restricting to $\gamma\left[0, t_{0}\right)$ we see that $F(\gamma(t))$ converges to some point $q \in \Omega$ as $t$ increases to $t_{0}$. Since $S \subset D \times \Omega$ is closed we must have $(p, q) \in S$, contradicting the statement that $p \notin D^{\prime}$.

Remarks. Theorem 2.1.1 can be deduced from Calabi [Ca]. Using the canonical embedding $\Psi_{G}: G \rightarrow \mathbb{P}\left(H^{2}(G)^{*}\right)$, by the existence and uniqueness theorems of [Ca] one can analytically continue holomorphic isometries along paths. Global extension can be deduced using the diastasis $\delta$ as defined and developed in [Ca], noting that $\delta_{D}\left(z, x_{0}\right)=$ $\log K_{D}^{\prime}(z, z)$. For a proof of interior extension using [Ca] we refer the reader to Mok $[\mathrm{Mk} 5,(2.3)]$. [Ca] does not however apply to boundary extension, since $\partial G$ essentially disappears under $\Psi_{G}$. Here interior extension is presented as a natural intermediate outcome of our direct method which at the same time yields boundary extension. 
For boundary extension results on bounded domains we have

Theorem 2.1.2. Let $D \Subset \mathbb{C}^{n}$ and $\Omega \Subset \mathbb{C}^{N}$ be bounded domains. Let $x_{0} \in D, \lambda$ be a positive real number and $f:\left(D, \lambda d s_{D}^{2} ; x_{0}\right) \rightarrow\left(\Omega, d s_{\Omega}^{2} ; f\left(x_{0}\right)\right)$ be a germ of holomorphic isometry. Suppose furthermore that the Bergman kernel $K_{D}(z, w)$ extends as a meromorphic function of $(z, \bar{w})$ to a neighborhood of $\bar{D} \times D$ and $K_{\Omega}(\zeta, \xi)$ extends as a meromorphic function of $(\zeta, \bar{\xi})$ to a neighborhood of $\bar{\Omega} \times \Omega$. Then there exist a neighborhood $D^{\sharp}$ of $\bar{D}$ and a neighborhood $\Omega^{\sharp}$ of $\bar{\Omega}$ such that the germ of $\operatorname{Graph}(f) \subset D \times \Omega$ at $\left(x_{0}, f\left(x_{0}\right)\right)$ extends to an irreducible complex-analytic subvariety $S^{\sharp}$ of $D^{\sharp} \times \Omega^{\sharp}$. If $\left(\Omega, d s_{\Omega}^{2}\right)$ is complete as a Kähler manifold, then $S:=S^{\sharp} \cap(D \times \Omega)$ is the graph of a holomorphic isometric embedding $F:\left(D, \lambda d s_{D}^{2}\right) \rightarrow\left(\Omega, d s_{\Omega}^{2}\right)$. If furthermore $\left(D, d s_{D}^{2}\right)$ is complete, then $F: D \rightarrow \Omega$ is proper.

Proof. We refer to the proof of Theorem 2.1.1 and use the same notation. Under the present hypothesis the domain of definition of the equations defining $V^{\prime} \subset D \times \Omega$, viz., the functional equations $\left(\mathbf{L}_{w}^{i}\right)$ for $w \in D_{\epsilon}$ and for $1 \leq i \leq n$, can be extended from $D \times \Omega$ to $D^{\sharp} \times \Omega^{\sharp}$. Denote by $V^{\prime \sharp}$ the common solution set of the extension of the functional equations $\left(\mathbf{L}_{w}^{i}\right)$ thus defined. On the other hand, from the formula for $h_{1}=$ $h_{\alpha}=h_{\eta, z_{0}}, \alpha \in \mathbf{A}$, given in (2) and (4) in the proof of Lemma 1.1.2, under the present assumption, each $h_{\alpha}$ can be extended from $\Omega$ to $\Omega^{\sharp}$ as a meromorphic function $h_{\alpha}^{\sharp}$. Recall that $E \subset \Omega$ is the common zero set of $h_{\alpha}, \alpha \in \mathbf{A}$. Defining $E^{\sharp}$ to be the common zero set of the meromorphic functions $h_{\alpha}^{\sharp}, \alpha \in \mathbf{A}$, on $\Omega^{\sharp}$, and writing $S^{\sharp} \subset D^{\sharp} \times \Omega^{\sharp}$ for the irreducible component of $V^{\prime \sharp} \cap\left(D^{\sharp} \times E^{\sharp}\right)$ containing Graph $(f)$, we infer that $S^{\sharp}$ furnishes an extension of Graph $(f)$ from $D \times \Omega$ to $D^{\sharp} \times \Omega^{\sharp}$ and $S^{\sharp} \cap(D \times \Omega)=S=\operatorname{Graph}(f)$, by the proof of Corollary 1.2.1. By Theorem 2.1.1, $S \subset D \times \Omega$ is the graph of a holomorphic isometric embedding $F:\left(D, \lambda d s_{D}^{2}\right) \rightarrow\left(\Omega, d s_{\Omega}^{2}\right)$.

It remains to prove that $F: D \rightarrow \Omega$ is proper whenever $\left(D, d s_{D}^{2}\right)$ is complete. Suppose otherwise; then there exists $b:=(p, y) \in S^{\sharp}-S$ such that $p \in \partial D$ and $y \in \Omega$. Let $W$ be a neighborhood of $(p, y)$ on $S^{\sharp}$ such that $W \subset D^{\sharp} \times \Omega$, and denote by $\rho: \widetilde{W} \rightarrow W$ a desingularization of $W$. Let $\eta=\left(x_{1}, y_{1}\right) \in W \cap \operatorname{Graph}(F)$ and denote by $\widetilde{\eta} \in \widetilde{W}$ the unique point lying over $\left(x_{1}, y_{1}\right)$. Let $\widetilde{b} \in \widetilde{W}$ be any point such that $\rho(\tilde{b})=b$. Let $\gamma:[0,1] \rightarrow \widetilde{W}$ be any smooth curve on $\widetilde{W}$ such that $\gamma(0)=\widetilde{\eta}$ and $\gamma(1)=\widetilde{b}$. Define $\gamma_{1}:[0,1] \rightarrow D^{\sharp}$ and $\gamma_{2}:[0,1] \rightarrow \Omega$ by $\gamma_{i}(t)=\pi_{i}(\rho(\gamma(t)), i=1,2$, where $\pi_{1}: D^{\sharp} \times \Omega \rightarrow D^{\sharp}$ and $\pi_{2}: D^{\sharp} \times \Omega \rightarrow \Omega$ are the canonical projections. Let $0<t^{\mathrm{b}} \leq 1$ be the first point such that $\gamma_{1}\left(t^{b}\right) \in \partial D$ and write $x^{b}:=\gamma_{1}\left(t^{b}\right) \in \partial D$ and $y^{b}:=\gamma_{2}\left(t^{b}\right) \in \Omega$. Then $\left.\gamma_{1}\right|_{\left[0, t^{b}\right]}:\left[0, t^{b}\right] \rightarrow D^{\sharp}$ joins $\gamma_{1}(0)=x_{1}$ to $x^{b}$ with $\gamma_{1}(t) \in D$ for $0 \leq t<t^{b}$. On the other hand, $\left.\gamma_{2}\right|_{\left[0, t^{b}\right]}:\left[0, t^{b}\right] \rightarrow \Omega$ joins $\gamma_{2}(0)=y_{1}$ to $\gamma_{2}\left(t^{b}\right)=y^{b}$. Since $\gamma$ is smooth, $\left.\gamma_{2}\right|_{\left[0, t^{b}\right]}$ is of finite length. Clearly $F\left(\gamma_{1}(t)\right)=\gamma_{2}(t)$ whenever $0 \leq t<t^{b}$. Since $F$ is an isometry, $\left.\gamma_{1}\right|_{\left[0, t^{b}\right)}$ must be of finite length with respect to the Bergman metric $d s_{D}^{2}$. However, $\left.\gamma_{1}\right|_{\left[0, t^{\mathrm{b}}\right]}$ is a smooth curve joining $x_{1} \in D$ to $x^{\mathrm{b}} \in \partial D$, and hence $\left.\gamma_{1}\right|_{\left[0, t^{\mathrm{b}}\right)}$ must be of infinite length on the complete Kähler manifold $\left(D, d s_{D}^{2}\right)$. This contradiction proves that $F: D \rightarrow \Omega$ is proper, and the proof is complete.

Remarks. In Theorem 1.1.1 we deal with boundary extension for germs of holomorphic isometries $f:\left(D, \lambda d s_{D}^{2} ; 0\right) \rightarrow\left(\Omega, d s_{\Omega}^{2} ; 0\right)$ between bounded complete circular domains 
with base points at 0 . For arbitrary base points $x_{0} \in D$ and $y_{0}=f\left(x_{0}\right) \in \Omega$, Theorem 2.1.2 applies provided that $t D \subset D$ and $t \Omega \subset \Omega$ whenever $0<t<1$. To see this, by Lemma 1.1.1, $K_{D, w}(z)=K_{D}(z, w)$ extends holomorphically to some neighborhood $D^{\sharp}$ of $\bar{D}$ whenever $w$ is sufficiently close to $x_{0}$, and the analogue holds true for $K_{\Omega, \xi}(\zeta)=$ $K_{\Omega}(\zeta, \xi)$ whenever $\xi$ is sufficiently close to $y_{0}$, so that Theorem 2.1.2 is applicable.

\subsection{Generalizations to relatively compact subdomains of complex manifolds}

We consider more general extensions of germs of holomorphic isometries on complex manifolds equipped with Bergman metrics. First of all, we introduce some terminology.

Definition 2.2.1. Let $X$ be a complex manifold and denote by $\omega_{X}$ its canonical line bundle. Suppose the Hilbert space $H^{2}\left(X, \omega_{X}\right)$ of square-integrable holomorphic $n$-forms on $X$ has no base points, and denote by $\mathcal{K}_{X}(z, w)$ the Bergman kernel form on $X$. Regarding $\mathcal{K}_{X}(z, z)$ as a Hermitian metric $h$ on the anti-canonical line bundle $\omega_{X}^{*}$, we denote by $\beta_{X} \geq 0$ the curvature form of the dual metric $h^{*}$ on $\omega_{X}$, and write $d s_{X}^{2}$ for the corresponding semi-Kähler metric on $X$. We say that $\left(X, d s_{X}^{2}\right)$ is a Bergman manifold whenever $d s_{X}^{2}$ is positive definite. If furthermore the canonical map $\Psi_{X}: X \rightarrow \mathbb{P}\left(H^{2}\left(X, \omega_{X}\right)^{*}\right)$ is an embedding, we call $\left(X, d s_{X}^{2}\right)$ a canonically embeddable Bergman manifold.

For a bounded domain $D \Subset \mathbb{C}^{n}$, we have $\mathcal{K}_{D}(z, w)=K_{D}(z, w)\left(\frac{i}{2} d z^{1} \wedge \overline{d w^{1}}\right) \wedge \cdots \wedge$ $\left(\frac{i}{2} d z^{n} \wedge \overline{d w^{n}}\right)$. Our extension results generalize to canonically embeddable Bergman manifolds, including bounded domains on Stein manifolds:

Theorem 2.2.1. Let $D$ and $\Omega$ be canonically embeddable Bergman manifolds. Let $D \Subset M$ (resp. $\Omega \Subset Q$ ) be a realization of $D$ (resp. $\Omega$ ) as a relatively compact domain on a complex manifold $M$ (resp. $Q)$ such that the Bergman kernel form $\mathcal{K}_{D}(z, w)\left(\right.$ resp. $\left.\mathcal{K}_{\Omega}(\zeta, \xi)\right)$ extends meromorphically in $(z, \bar{w})$ to $M \times D$ (resp. in $(\zeta, \bar{\xi})$ to $Q \times \Omega)$. Then the analogue of Theorem 2.1.2 holds true with $M$ replacing $D^{\sharp}$ and $Q$ replacing $\Omega^{\sharp}$.

Proof. Let $\mu$ be a square-integrable holomorphic $n$-form on $D$ such that $\mu\left(x_{0}\right) \neq 0$, and $v$ be a square-integrable holomorphic $N$-form on $\Omega$ such that $v\left(f\left(x_{0}\right)\right) \neq 0$. For $m>0$, write $\epsilon_{m}=(\sqrt{-1})^{m^{2}}$ so that $\epsilon_{m} \alpha \wedge \bar{\alpha} \geq 0$ for any $(m, 0)$-covector $\alpha$ on an $m$-dimensional complex manifold. Define $K_{D}^{\mathrm{b}}(z, w)$ on $D \times D$, resp. $K_{\Omega}^{\mathrm{b}}(\zeta, \xi)$ on $\Omega \times \Omega$, by

$$
\mathcal{K}_{D}(z, w)=K_{D}^{b}(z, w)\left(\epsilon_{n} \mu(z) \wedge \overline{\mu(w)}\right), \quad \mathcal{K}_{\Omega}(\zeta, \xi)=K_{\Omega}^{b}(\zeta, \xi)\left(\epsilon_{N} v(\zeta) \wedge \overline{v(\xi)}\right) .
$$

Using $K_{D}^{b}(z, w)$, resp. $K_{\Omega}^{b}(\zeta, \xi)$, in place of $K_{D}^{\prime}(z, w)$, resp. $K_{\Omega}^{\prime}(\zeta, \xi)$, Theorems 2.1 .1 and 2.1.2 generalize as follows. Let $\sigma_{0} \in H^{2}\left(D, \omega_{D}\right)$ be such that the $(n, n)$-vector $\epsilon_{n} \sigma\left(x_{0}\right) \wedge \overline{\sigma\left(x_{0}\right)}$ is maximized among square-integrable holomorphic $n$-forms of unit norm by $\sigma=\sigma_{0}$. Then $\sigma\left(x_{0}\right)=0$ for any $\sigma \perp \sigma_{0}$. Complete $\sigma_{0}$ to an orthonormal basis $\left(\sigma_{i}\right)_{i=0}^{\infty}$ of $H^{2}\left(D, \omega_{D}\right)$. Choosing $\mu=\sigma_{0}$, we have

$$
K_{D}^{\mathrm{b}}(z, z)=\sum_{i=0}^{\infty}\left|\frac{\sigma_{i}(z)}{\sigma_{0}(z)}\right|^{2}=1+\sum_{i=1}^{\infty}\left|\frac{\sigma_{i}(z)}{\sigma_{0}(z)}\right|^{2} .
$$


Similarly let $\left(\tau_{i}\right)_{i=0}^{\infty}$ be an orthonormal basis of $H^{2}\left(\Omega, \omega_{\Omega}\right)$ adapted to $y_{0}=f\left(x_{0}\right)$ defined in exactly the same way. Choosing $v=\tau_{0}$, we have

$$
K_{\Omega}^{\mathrm{b}}(\zeta, \zeta)=\sum_{i=0}^{\infty}\left|\frac{\tau_{i}(\zeta)}{\tau_{0}(\zeta)}\right|^{2}=1+\sum_{i=1}^{\infty}\left|\frac{\tau_{i}(\zeta)}{\tau_{0}(\zeta)}\right|^{2}
$$

Then $K_{D}^{\mathrm{b}}(z, w)$, resp. $K_{\Omega}^{\mathrm{b}}(\zeta, \xi)$, plays the role of $K_{D}^{\prime}(z, w)$, resp. $K_{\Omega}^{\prime}(\zeta, \xi)$, in Theorem 2.1.1, and by the analogues of (2) and (3) in the proof of Theorem 2.1.1 we have

$$
\begin{aligned}
\sqrt{-1} \partial \bar{\partial} \log K_{\Omega}^{\mathrm{b}}(f(z), f(z)) & =\lambda \sqrt{-1} \partial \bar{\partial} \log K_{D}^{\mathrm{b}}(z, z), \\
\log K_{\Omega}^{\mathrm{b}}(f(z), f(z)) & =\lambda \log K_{D}^{\mathrm{b}}(z, z),
\end{aligned}
$$

and the proofs there carry over with minor modifications to yield Theorem 2.2.1.

Remarks. For a bounded symmetric domain $G \subset N$ embedded in its compact dual $N$ by the Borel embedding, $\mathcal{K}_{G}(z, w)$ extends meromorphically in $(z, \bar{w})$ to $N$ (cf. Lemma 1.3.1). Thus, Theorem 2.2.1 implies Theorem 1.3.1.

\section{Examples of holomorphic isometries with respect to the Bergman metric}

\subsection{Totally geodesic examples on bounded symmetric domains}

The first examples of non-equidimensional holomorphic isometric embeddings $f$ : $D \rightarrow \Omega$ up to normalizing constants with respect to the Bergman metric are given by holomorphic totally geodesic embeddings from an irreducible bounded symmetric domain into any bounded symmetric domain, such as the embedding of the Poincaré disk into the complex unit ball $B^{n}, n \geq 2$, given by $f(z)=(z, 0)$, or the diagonal map into the polydisk $\Delta^{n}, n \geq 2$, given by $f_{n}(z)=(z, \ldots, z)$. More generally, if $\Omega$ is a bounded symmetric domain of rank $r \geq 1$, then, up to automorphisms of $\Omega$, there are exactly $r$ such maps, obtained from a maximal polydisk $P \subset \Omega$, where $P \cong \Delta^{r}$, and $f: \Delta \rightarrow \Omega$ is given by composing the diagonal map $f_{k}: \Delta \rightarrow \Delta^{k}$ with the standard embedding $\Delta^{k} \times\{0\} \subset \Delta^{r} \cong P \subset \Omega, 1 \leq k \leq r$.

Totally geodesic holomorphic embeddings $f: D \rightarrow \Omega$ from irreducible bounded symmetric domains into bounded symmetric domains have been classified by Satake [Sa] and Ihara [Ih]. As higher-dimensional examples write $M(p, q)$ for the complex vector space of $p$-by- $q$ matrices with complex entries, and recall that the domain $D_{p, q}^{\mathrm{I}} \subset$ $M(p, q)$ consists of matrices $Z$ satisfying $I-\bar{Z}^{t} Z>0$. Let $M_{a}(n) \subset M(n, n)$, resp. $M_{S}(n) \subset M(n, n)$, be the complex vector subspace consisting of skew-symmetric, resp. symmetric, matrices. Define $D_{n}^{\mathrm{II}}:=D_{n, n}^{\mathrm{I}} \cap M_{a}(n)$ and $D_{n}^{\mathrm{III}}:=D_{n, n}^{\mathrm{I}} \cap M_{s}(n)$. Then $D_{p, q}^{\mathrm{I}} \Subset M(p, q)$, resp. $D_{n}^{\mathrm{II}} \Subset M_{a}(n)$, resp. $D_{n}^{\mathrm{III}} \Subset M_{s}(n)$, are classical symmetric domains of type I, resp. II, resp. III, in their Harish-Chandra realizations, and the inclusions $D_{n}^{\mathrm{II}} \subset D_{n, n}^{\mathrm{I}}$ and $D_{n}^{\mathrm{III}} \subset D_{n, n}^{\mathrm{I}}$ are totally geodesic. They extend to holomorphic embeddings $M_{a}(n) \subset M(n, n)$ and $M_{s}(n) \subset M(n, n)$. More generally, using the characterization of totally geodesic submanifolds on a Riemannian symmetric manifold in terms of 
Lie triple systems (cf. Helgason [He, §7, p. $224 \mathrm{ff}$.$] ), the Borel embedding between dual$ pairs of Hermitian symmetric spaces, and Harish-Chandra coordinates (cf. Wolf [Wo]), we have the following summary of basic facts for which the proof is omitted.

Proposition 3.1.1. Let $(D, h)$ and $(\Omega, g)$ be Hermitian symmetric manifolds of the noncompact type and denote by $\left(M, h_{c}\right)$, resp. $\left(Q, g_{c}\right)$, the compact dual of $D$, resp. $\Omega$. Identify $D$ and $\Omega$ as bounded symmetric domains $D \Subset \mathbb{C}^{n}, \Omega \Subset \mathbb{C}^{N}$ in their HarishChandra realizations, so that $D \Subset \mathbb{C}^{n} \subset M$ and $\Omega \Subset \mathbb{C}^{N} \subset Q$, where $D \subset M$ and $\Omega \subset Q$ are given by the Borel embedding. Let $F: D \rightarrow(\Omega, g)$ be a holomorphic totally geodesic embedding. Then $F$ extends to a holomorphic totally geodesic embedding $\Phi: M \rightarrow\left(Q, g_{c}\right)$. As a consequence, $\operatorname{Graph}(F) \subset D \times \Omega$ extends to a complex submanifold $S \subset M \times Q$. When $D$ is irreducible, $F$ is a holomorphic isometry up to a normalizing constant. If $F(0)=0$, then $F$ is the restriction of a linear map $\Lambda: \mathbb{C}^{n} \rightarrow \mathbb{C}^{N}$.

Let $D \Subset \mathbb{C}^{n}$ be an irreducible bounded symmetric domain in its Harish-Chandra realization. Denote by $\pi: L \rightarrow D$ the anti-canonical line bundle on $D$. Writing $\left(z_{1}, \ldots, z_{n}\right)$ for the Harish-Chandra coordinates on $D$, for $t \in \mathbb{C}$ the $n$-vector $t \frac{\partial}{\partial z_{1}} \wedge \cdots \wedge \frac{\partial}{\partial z_{n}}$ at any $z \in D$ is identified with $(z, t)$, giving a trivialization $L \cong D \times \mathbb{C}$. The action of $\operatorname{Aut}(D)$ on $D$ induces an action on $L$, and $\pi: L \rightarrow D$ is equipped with an $\operatorname{Aut}(D)$-invariant Hermitian metric $h$. Thus, given any $z \in D$ and $\gamma \in \operatorname{Aut}(D)$ we have $f_{*}\left(\frac{\partial}{\partial z_{1}} \wedge \cdots \wedge \frac{\partial}{\partial z_{n}}\right)=J_{\gamma}(z) \cdot \frac{\partial}{\partial z_{1}} \wedge \cdots \wedge \frac{\partial}{\partial z_{n}}$, where $J_{\gamma}(z)=\operatorname{det}(d \gamma(z))$ is the Jacobian determinant of $\gamma$, and the action of $\operatorname{Aut}(D)$ on $L$ is given by $\Phi(\gamma)(z, t)=\left(\gamma(z), J_{\gamma}(z) \cdot t\right)$. On $L$ we have the open subset $\Omega \subset L$ consisting of all $n$-vectors $\eta$ of length $<1$ with respect to $h$. By the Schwarz Lemma, the volume form of the Bergman metric $d s_{D}^{2}$ is bounded from below by a constant multiple of the Euclidean volume form, so that $\Omega \subset D \times \Delta(R) \Subset \mathbb{C}^{n+1}$ for some $R>0, \Delta(R)$ being the disk of radius $R$ centered at 0 . Let now $\alpha$ be a positive real number. We define $L^{\alpha}:=D \times \mathbb{C}$ set-theoretically to be the same as $L$, but regard $\pi: L^{\alpha} \rightarrow D$ as being equipped with the Hermitian metric $h^{\alpha}$, where, writing $e$ for the basis of $L \cong D \times \mathbb{C}$ corresponding to $D \times\{1\}$, and writing $e^{\alpha}$ for the basis of $L^{\alpha} \cong D \times \mathbb{C}$ corresponding to $D \times\{1\}$, we have $\left\|e^{\alpha}\right\|_{h^{\alpha}}=\|e\|_{h}^{\alpha}$. We define $\Omega_{\alpha} \subset L^{\alpha}$ to consist of vectors $\eta$ of length $<1$ with respect to $h_{\alpha}, \Omega_{\alpha} \Subset \mathbb{C}^{n+1}$. Thus, $\Omega_{\alpha} \subset L^{\alpha}$ is the unit disk bundle of $\pi: L^{\alpha} \rightarrow D$ with respect to a Hermitian metric of strictly negative curvature on $L^{\alpha}=D \times \mathbb{C}$, so that every boundary point $b \in \partial \Omega_{\alpha}-\partial D$ is strictly pseudoconvex ( $D$ being identified with $D \times\{0\}$ ). With this set-up we prove

Proposition 3.1.2. Let $\alpha>0$ and $f: D \rightarrow \Omega_{\alpha}$ be the embedding given by $f(z)=$ $(z, 0)$. Then $f:\left(D, \lambda d s_{D}^{2}\right) \rightarrow\left(\Omega_{\alpha}, d s_{\Omega_{\alpha}}^{2}\right)$ is a totally geodesic holomorphic isometric embedding for $\lambda=1+\alpha$. Furthermore, $\left(\Omega_{\alpha}, d s_{\Omega_{\alpha}}^{2}\right)$ is a complete Kähler manifold.

Proof. Since $D$ is simply connected, for $\gamma \in \operatorname{Aut}(D)$ a holomorphic $\log$ arithm $\log J_{\gamma}(z)$ can be defined for the Jacobian determinant $J_{\gamma}(z)=\operatorname{det}(d \gamma(z))$, and the mapping $\Psi_{\gamma}(z, \eta)=\left(\gamma(z), \exp \left(\alpha \log J_{\gamma}(z)\right)\right.$ defines an automorphism of $\pi: L^{\alpha} \rightarrow D$ as a holomorphic line bundle which preserves the Hermitian metric $h^{\alpha}$. Identify $D$ as the zero section of $\pi: L^{\alpha} \rightarrow D$ and denote by $H \subset \operatorname{Aut}\left(\Omega_{\alpha}\right)$ the subgroup which leaves $D$ invariant as a set. Since $H$ acts transitively on $D \subset L^{\alpha}$ by means of $\Psi_{\gamma}, \gamma \in \operatorname{Aut}(D)$, the 
restriction of the Bergman kernel $\Omega_{\alpha}$ to $D$ can be computed from a single point, giving

$$
K_{\Omega_{\alpha}}((z, 0),(z, 0))=|\operatorname{det}(d \gamma(0))|^{-2(1+\alpha)} K_{\Omega_{\alpha}}(0,0),
$$

where $\gamma$ is an automorphism of $D$ such that $\gamma(0)=z$. On the other hand,

$$
K_{D}(z, z)=|\operatorname{det}(d \gamma(0))|^{-2} K_{D}(0,0) .
$$

Comparing (1) and (2) we conclude that

$$
K_{\Omega_{\alpha}}((z, 0),(z, 0))=c_{\alpha} \cdot K_{D}(z, z)^{1+\alpha}
$$

for $c_{\alpha}>0$. Writing $\varphi_{D}(z):=K_{D}(z, z)$ and $\varphi_{\Omega_{\alpha}}(\zeta)=K_{\Omega_{\alpha}}(\zeta, \zeta)$, from (3) we deduce

$$
\begin{aligned}
\left.\sqrt{-1} \partial \bar{\partial} \log \varphi_{\Omega_{\alpha}}\right|_{D} & =(1+\alpha) \sqrt{-1} \partial \bar{\partial} \log \varphi_{D}, \quad \text { i.e. }, \\
f^{*} d s_{\Omega_{\alpha}}^{2} & =(1+\alpha) d s_{D}^{2},
\end{aligned}
$$

as desired. Since $D \subset \Omega_{\alpha}$ is the fixed point set of the circle group $S^{1}$ acting by $\left(e^{i \theta} ;(z, t)\right) \mapsto\left(z, e^{i \theta} t\right), D \subset \Omega_{\alpha}$ is totally geodesic with respect to $d s_{\Omega_{\alpha}}^{2}$. It remains to prove that $\left(\Omega_{\alpha}, d s_{\Omega_{\alpha}}^{2}\right)$ is complete, for which it suffices to show that, given any sequence $\left(x_{j}\right)_{j=1}^{\infty}$ of points approaching $b \in \partial \Omega_{\alpha}, d\left(0, x_{j}\right)$ must diverge to $\infty$ as $j \rightarrow \infty$. Let $x \in \Omega_{\alpha}$ be any point and $\gamma:[0,1] \rightarrow \Omega_{\alpha}$ be a piecewise $\mathcal{C}^{1}$-curve joining 0 to $x$. Then $\pi \circ \gamma:[0,1] \rightarrow D$ is a piecewise $\mathcal{C}^{1}$-curve joining 0 to $\pi(x) \in D$. Denote by $d_{D}(\cdot, \cdot)$, resp. $d_{\Omega_{\alpha}}(\cdot, \cdot)$, the distance function for the Kähler manifold $\left(D, d s_{D}^{2}\right)$, resp. $\left(\Omega_{\alpha}, d s_{\Omega_{\alpha}}^{2}\right)$. For a complex manifold $X$ we denote by $\kappa_{X}$ its Carathéodory pseudo-metric, which is an $\operatorname{Aut}(X)$-invariant continuous complex Finsler pseudo-metric, and by $\delta_{X}(\cdot, \cdot)$ the pseudodistance function of $\left(X, \kappa_{X}\right)$. When $X$ is a bounded domain, $\kappa_{X}$ is a metric, and $\delta_{X}(\cdot, \cdot)$ is a distance function. Since $D$ is homogeneous, any two $\operatorname{Aut}(D)$-invariant continuous complex Finsler metrics are equivalent to each other, in particular $\delta_{D}(\cdot, \cdot) \geq c \cdot d_{D}(\cdot, \cdot)$ for some constant $c>0$. By the distance-decreasing property of the Carathéodory metric, $\delta_{D}(\pi(x), 0) \leq \delta_{\Omega_{\alpha}}(x, 0)$. Since the Bergman metric on any bounded domain dominates the Carathéodory metric, $d_{\Omega_{\alpha}}(x, 0) \geq \delta_{\Omega_{\alpha}}(x, 0) \geq \delta_{D}(\pi(x), 0) \geq c \cdot d_{D}(\pi(x), 0)$. Let $\left(x_{j}\right)_{j=1}^{\leq \infty}$ be a discrete sequence on $\Omega_{\alpha}$ converging to $b \in \partial D \subset \partial \bar{\Omega}_{\alpha}$. Then $d_{\Omega_{\alpha}}\left(x_{j}, 0\right) \geq$ $c \cdot d_{D}\left(\pi\left(x_{j}\right), 0\right) \rightarrow \infty$ since $\left(D, d s_{D}^{2}\right)$ is complete. On the other hand, if $b \in \partial \Omega_{\alpha}-\partial D$, then $b$ is a smooth strictly pseudoconvex boundary point of $\Omega_{\alpha}$. By a standard localization argument, $\delta_{\Omega_{\alpha}}\left(x_{j}, 0\right) \rightarrow \infty$ as $j \rightarrow \infty$, and $d_{\Omega_{\alpha}}\left(x_{j}, 0\right) \geq \delta_{\Omega_{\alpha}}\left(x_{j}, 0\right) \rightarrow \infty$, proving that $\left(\Omega_{\alpha}, d s_{\Omega_{\alpha}}^{2}\right)$ is complete, as desired.

\subsection{Examples of holomorphic isometric embeddings of the Poincare disk into the polydisk}

Motivated by Clozel-Ullmo [CU], our first aim was to study germs of holomorphic isometries $f:(D ; 0) \rightarrow(\Omega ; 0)$ between bounded symmetric domains. In particular, in relation to the case where $D$ is the unit disk $\Delta$ and $\Omega$ is the polydisk $\Delta^{p}$, it was conjectured in [CU, Conjecture 2.2] that for any positive integer $q$, every germ of holomorphic isometry 
$f:\left(\Delta, q d s_{\Delta}^{2} ; 0\right) \rightarrow\left(\Delta^{p}, d s_{\Delta^{p}}^{2} ; 0\right)$ is necessarily totally geodesic. We can a priori allow the normalizing (positive) real constant $\lambda$ to be arbitrary. By Theorem 1.3.1, $f$ necessarily extends to a proper holomorphic embedding $F: \Delta \rightarrow \Delta^{p}$ whose graph extends to an irreducible affine-algebraic subvariety $S^{\sharp} \subset \mathbb{C} \times \mathbb{C}^{p}$. It follows readily that $\lambda$ is necessarily a positive integer $q$. (This can be seen by comparing Bergman kernels via a local holomorphic extension $F^{b}$ across a general boundary point $b \in \partial \Delta$.)

Let $D$ and $\Omega$ be bounded symmetric domains, and $F, \widetilde{F}: D \rightarrow \Omega$ be holomorphic maps. We say that $F$ and $\widetilde{F}$ are congruent whenever there exists $\varphi \in \operatorname{Aut}(D)$ and $\psi \in$ $\operatorname{Aut}(\Omega)$ such that $\widetilde{F}=\psi \circ F \circ \varphi$, and incongruent otherwise. Concerning holomorphic isometric embeddings $F:\left(\Delta, q d s_{\Delta}^{2}\right) \rightarrow\left(\Delta^{p}, d s_{\Delta^{p}}^{2}\right)$, we have

Theorem 3.2.1. For every positive integer $p>1$ there exists a holomorphic isometric embedding $F:\left(\Delta, d s_{\Delta}^{2}\right) \rightarrow\left(\Delta^{p}, d s_{\Delta^{p}}^{2}\right), F=\left(F_{1}, \ldots, F_{p}\right)$, where each component $F_{k}, 1 \leq k \leq p$, is nonconstant, such that $F$ is not totally geodesic. In particular, Conjecture 2.2 of Clozel-Ullmo [CU] is false. Furthermore, for $p \geq 3$ there exists a realanalytic 1-parameter family of mutually incongruent holomorphic isometric embeddings $F_{t}:\left(\Delta, d s_{\Delta}^{2}\right) \rightarrow\left(\Delta^{p}, d s_{\Delta^{p}}^{2}\right), t \in \mathbb{R}$.

We start with an example of a holomorphic isometric embedding of the Poincaré disk into the bi-disk. The unit disk is conformally equivalent to the upper half-plane $\mathcal{H}$. For $\tau \in \mathcal{H}$, $\tau=\rho e^{i \varphi}$, where $\rho>0,0<\varphi<\pi$, write $\sqrt{\tau}=\sqrt{\rho} e^{i \varphi / 2}$. Then we have

Lemma 3.2.1. Equip $\mathcal{H}$ with the Poincaré metric $d s_{\mathcal{H}}^{2}=2 \operatorname{Re} \frac{d \tau \otimes d \bar{\tau}}{2(\operatorname{Im} \tau)^{2}}$ of constant Gaussian curvature -1 , and $\mathcal{H}^{2}$ with the product metric. Then the proper holomorphic map $f: \mathcal{H} \rightarrow \mathcal{H}^{2}$ given by $f(\tau)=(\sqrt{\tau}, i \sqrt{\tau})$ is a holomorphic isometric embedding.

Proof. Let $\omega_{\mathcal{H}}$, resp. $\omega_{\mathcal{H}}$, be the Kähler forms of the chosen canonical Kähler metrics on $\mathcal{H}$, resp. $\mathcal{H}^{2}$. Writing $\tau=s+i t, \sqrt{\tau}=\alpha+i \beta$, where $s, t, \alpha$ and $\beta$ are real, we have

$$
\begin{aligned}
\omega_{\mathcal{H}} & =\sqrt{-1} \partial \bar{\partial}(-2 \log t)=\sqrt{-1} \frac{d \tau \wedge d \bar{\tau}}{2 t^{2}}, \\
f^{*} \omega_{\mathcal{H}^{2}} & =-2 \sqrt{-1} \partial \bar{\partial}(\log (\operatorname{Im}(\sqrt{\tau}))+\log (\operatorname{Im}(i \sqrt{\tau}))) \\
& =-2 \sqrt{-1} \partial \bar{\partial} \log (\operatorname{Im}(\sqrt{\tau}) \cdot \operatorname{Im}(i \sqrt{\tau})), \\
\operatorname{Im}(\sqrt{\tau}) \cdot \operatorname{Im}(i \sqrt{\tau}) & =\beta \alpha=\frac{1}{2} \operatorname{Im}\left(\left(\alpha^{2}-\beta^{2}\right)+2 i \alpha \beta\right)=\frac{1}{2} \operatorname{Im}(\tau)=\frac{t}{2}, \\
f^{*} \omega_{\mathcal{H}^{2}} & =-2 \sqrt{-1} \partial \bar{\partial} \log (t / 2)=\sqrt{-1} \partial \bar{\partial}(-2 \log t)=\omega_{\mathcal{H}} .
\end{aligned}
$$

In other words, $f:\left(\mathcal{H}, d s_{\mathcal{H}}^{2}\right) \rightarrow\left(\mathcal{H}, d s_{\mathcal{H}}^{2}\right) \times\left(\mathcal{H}, d s_{\mathcal{H}}^{2}\right)$ is a holomorphic isometry. It is an embedding since the function $\sqrt{\tau}$ is already injective on $\mathcal{H}$.

For $\tau \in \mathcal{H}, \tau=\rho e^{i \varphi}$, and an integer $p \geq 2$, write $\tau^{1 / p}=\rho^{1 / p} e^{i \varphi / p}$. Then we have

Proposition 3.2.1. Let $p \geq 2$ be a positive integer and $\gamma=e^{\pi i / p}$. Then the proper holomorphic mapping $f:\left(\overline{\mathcal{H}}, d s_{\mathcal{H}}^{2}\right) \rightarrow\left(\mathcal{H}, d s_{\mathcal{H}}^{2}\right)^{p}$ defined by

$$
f(\tau)=\left(\tau^{1 / p}, \gamma \tau^{1 / p}, \ldots, \gamma^{p-1} \tau^{1 / p}\right)
$$

is a holomorphic isometric embedding. 
Proof. Write $\tau^{1 / p}=r e^{i \theta}, 0<\theta<\pi / p$. Thus, $r^{p}=\rho, p \theta=\varphi$, and, for $0 \leq k \leq p-1$, $\operatorname{Im}\left(\gamma^{k} \tau^{1 / p}\right)=r \cdot \operatorname{Im}\left(e^{i(k \pi / p+\theta)}\right)$. Let $\tau_{k}$ be the standard coordinate of the $k$-th direct factor of $\mathcal{H}^{p}$, and write $\tau_{k}=s_{k}+i t_{k}$ with $s_{k}, t_{k}$ real. Then to prove the proposition it suffices to check that $f^{*}\left(\log t_{1}+\cdots+\log t_{p}\right)=a_{p}+\log t$ for some constant $a_{p}$. Now

$$
\begin{aligned}
f^{*}\left(\log t_{1}+\cdots+\log t_{p}\right) & =\log \left(\prod_{k=0}^{p-1} \operatorname{Im}\left(e^{i(k \pi / p+\theta)}\right)\right)+p \log r \\
& =\log \left(\prod_{k=0}^{p-1} \sin \left(\frac{k \pi}{p}+\theta\right)\right)+\log \rho .
\end{aligned}
$$

Writing $t=\operatorname{Im}(\tau)=\rho \sin \varphi=\rho \sin (p \theta)$, it remains to verify the following identity.

Lemma 3.2.2. Let $p \geq 2$ be a positive integer. Then the trigonometric identity

$$
\sin \theta \sin \left(\frac{\pi}{p}+\theta\right) \cdots \sin \left(\frac{(p-1) \pi}{p}+\theta\right)=c_{p} \sin (p \theta)
$$

holds true for some positive constant $c_{p}$.

Proof. Both sides of the displayed equation are trigonometric polynomials with exactly the same zero sets in $\theta$ consisting only of simple zeros. Hence, they must agree for some nonzero constant $c_{p}$, which is positive by substitution of some $\theta \in(0, \pi / p)$.

Proof of Theorem 3.2.1. The $p$-th root map as in Proposition 3.2.1 gives via the Cayley transform a holomorphic isometry $f_{p}:\left(\Delta, d s_{\Delta}^{2}\right) \rightarrow\left(\Delta^{p}, d s_{\Delta^{p}}^{2}\right)$. Here for the domain disk we use the Cayley transform $\iota: \mathcal{H} \rightarrow \Delta$ given by $z=\iota(\tau)=(\tau-i) /(\tau+i)$, and likewise the same map for each component of the target polydisk $\Delta^{p}$. This gives examples proving the first half of Theorem 3.2.1. We have $f_{p}(0)=0$, and $f_{p}$ is singular exactly at two points $1,-1 \in \partial \Delta$ on the boundary circle, with images $f_{p}(1)=(1, \ldots, 1)$ and $f_{p}(-1)=(-1, \ldots,-1)$. An example of a real-analytic 1-parameter family of holomorphic isometries $F_{t}:\left(\Delta, d s_{\Delta}^{2}\right) \rightarrow\left(\Delta^{p}, d s_{\Delta^{p}}^{2}\right)$ which are mutually incongruent to each other can be constructed from $f_{p-1}$ and $f_{2}$ as follows. Write $f_{2}(z)=(\alpha(z), \beta(z))$, $f_{p-1}(z)=\left(\gamma_{1}(z), \ldots, \gamma_{p-1}(z)\right)$, and let $\varphi \in \operatorname{Aut}(\Delta)$ be an arbitrary automorphism. Define $h: \Delta \rightarrow \Delta^{p}$ by $h(z):=\left(\alpha\left(\varphi\left(\gamma_{1}(z)\right)\right), \beta\left(\varphi\left(\gamma_{1}(z)\right), \gamma_{2}(z), \ldots, \gamma_{p-1}(z)\right)\right.$. Then $h=$ $g \circ f_{p-1}$, where $g: \Delta^{p-1} \rightarrow \Delta^{p}$ is given by $g\left(z_{1}, \ldots, z_{p-1}\right)=\left(f_{2}\left(\varphi\left(z_{1}\right)\right) ; z_{2}, \ldots, z_{p-2}\right)$. Thus, $g$ and hence $h$ are holomorphic isometries with respect to Bergman metrics. Observe that $\gamma_{1}(z)$, which corresponds to taking the $p$-th root in the coordinate $\tau=s+i t$ of the upper half-plane $\mathcal{H}$ (cf. Proposition 3.2.1), maps the lower semi-circle $S_{-}^{1}:=$ $\left\{e^{i \theta}:-\pi<\theta<0\right\}$ bijectively onto itself. (Note that the positive $s$-axis is mapped via $z=\iota(\tau)=(\tau-i) /(\tau+i)$ to $S_{-}^{1}$ since $\iota(1)=-i$.) Given any two distinct points $a, b \in S_{-}^{1}$, we can choose $\varphi \in \operatorname{Aut}(\Delta)$ such that $\varphi\left(\gamma_{1}(a)\right)=1$ and $\varphi\left(\gamma_{1}(b)\right)=-1$. Then, noting that in fact each component $\gamma_{k}, 1 \leq k \leq p-1$, of $f_{p-1}: \Delta \rightarrow \Delta^{p-1}$ can be analytically continued to no neighborhood of either 1 or $-1, h$ is singular precisely at the four distinct points $1,-1, a, b$. If we fix $a$ and let $b$ vary, we get holomorphic isometries $h_{b}:\left(\Delta, d s_{\Delta}^{2}\right) \rightarrow\left(\Delta^{p}, d s_{\Delta^{p}}^{2}\right)$ depending on $b$. For $b_{1} \neq b_{2}, h_{b_{1}}$ cannot be congruent 
to $h_{b_{2}}$ since the two sets $\left\{1,-1, a, b_{1}\right\}$ and $\left\{1,-1, a, b_{2}\right\}$ cannot be transformed to each other by any automorphism of $\Delta$. Letting $b$ vary on a connected component of $S_{-}^{1}-\{a\}$, we thus obtain a real-analytic 1-parameter family of mutually incongruent holomorphic isometries $F_{t}:\left(\Delta, d s_{\Delta}\right) \rightarrow\left(\Delta^{p}, d s_{\Delta p}^{2}\right)$, as desired.

3.3. An example of holomorphic isometric embedding of the unit disk into a Siegel upper half-plane

In this section we construct an example of a holomorphic isometric embedding from the Poincaré disk into some Siegel upper half-plane which does not arise from the examples given in 3.2. For a positive integer $g$, recall that $M_{S}(g)$ stands for the vector space of symmetric $g$-by- $g$ complex matrices, and $\mathcal{H}_{g} \subset M_{S}(g)$ for the Siegel upper half-plane of genus $g, \mathcal{H}_{g}:=\left\{\mathfrak{T} \in M_{s}(g): \operatorname{Im} \mathfrak{T}>0\right\}$. We have

Proposition 3.3.1. For $\zeta=\rho e^{i \varphi}, \rho>0,0<\varphi<\pi, n$ a positive integer, we write $\zeta^{1 / n}:=\rho^{1 / n} e^{i \varphi / n}$. Then the holomorphic mapping $G: \mathcal{H} \rightarrow M_{s}(3)$ defined by

$$
G(\tau)=\left[\begin{array}{ccc}
e^{\pi i / 6} \tau^{2 / 3} & \sqrt{2} e^{-\pi i / 6} \tau^{1 / 3} & 0 \\
\sqrt{2} e^{-\pi i / 6} \tau^{1 / 3} & i & 0 \\
0 & 0 & e^{\pi i / 3} \tau^{1 / 3}
\end{array}\right]
$$

maps $\mathcal{H}$ into $\mathcal{H}_{3}$, and $G:\left(\mathcal{H}, 2 d s_{\mathcal{H}}^{2}\right) \rightarrow\left(\mathcal{H}_{3}, d s_{\mathcal{H}_{3}}^{2}\right)$ is a holomorphic isometry.

Proof. Write $\tau^{1 / 3}=\alpha+i \beta$. We have $\tau=(\alpha+i \beta)^{3}=\left(\alpha^{3}-3 \alpha \beta^{2}\right)+i\left(3 \alpha^{2} \beta-\beta^{3}\right)$. In particular, $\operatorname{Im}(\tau)=3 \alpha^{2} \beta-\beta^{3}=\beta\left(3 \alpha^{2}-\beta^{2}\right)$. Note that $\tau \in \mathcal{H}$ if and only if $0<\operatorname{Arg}\left(\tau^{1 / 3}\right)<\pi / 3$, i.e., $0<\beta<\sqrt{3} \alpha$. We compute

$$
\begin{gathered}
e^{\pi i / 6} \tau^{2 / 3}=\left(\frac{\sqrt{3}}{2}+\frac{i}{2}\right)\left(\left(\alpha^{2}-\beta^{2}\right)+2 i \alpha \beta\right), \text { hence } \\
\operatorname{Im}\left(e^{\pi i / 6} \tau^{2 / 3}\right)=\frac{1}{2}\left(\alpha^{2}-\beta^{2}\right)+\sqrt{3} \alpha \beta \\
\sqrt{2} e^{-\pi i / 6} \tau^{1 / 3}=\sqrt{2}\left(\frac{\sqrt{3}}{2}-\frac{i}{2}\right)(\alpha+i \beta), \text { hence } \operatorname{Im}\left(\sqrt{2} e^{-\pi i / 6} \tau^{1 / 3}\right)=\frac{\sqrt{6}}{2} \beta-\frac{\sqrt{2}}{2} \alpha ; \\
e^{\pi i / 3} \tau^{1 / 3}=\left(\frac{1}{2}+\frac{\sqrt{3}}{2} i\right)(\alpha+i \beta), \text { hence } \operatorname{Im}\left(e^{\pi i / 3} \tau^{1 / 3}\right)=\frac{\sqrt{3}}{2} \alpha+\frac{\beta}{2} .
\end{gathered}
$$

Thus,

$$
\begin{aligned}
& \operatorname{det}(\operatorname{Im} G)=\operatorname{det}\left[\begin{array}{ccc}
\frac{1}{2}\left(\alpha^{2}-\beta^{2}\right)+\sqrt{3} \alpha \beta & \frac{\sqrt{6}}{2} \beta-\frac{\sqrt{2}}{2} \alpha & 0 \\
\frac{\sqrt{6}}{2} \beta-\frac{\sqrt{2}}{2} \alpha & 1 & 0 \\
0 & 0 & \frac{\sqrt{3}}{2} \alpha+\frac{\beta}{2}
\end{array}\right] \\
& =\left(-2 \beta^{2}+2 \sqrt{3} \alpha \beta\right)\left(\frac{\sqrt{3}}{2} \alpha+\frac{\beta}{2}\right)=\beta(\sqrt{3} \alpha-\beta)(\sqrt{3} \alpha+\beta)=\beta\left(3 \alpha^{2}-\beta^{2}\right)=\operatorname{Im} \tau .
\end{aligned}
$$


Write $\lambda:=\beta / \alpha, 0<\lambda<\sqrt{3}$. From the above, the determinant of the upper 2-by-2 matrix of $\operatorname{Im} G$ is positive. To check positivity of $\operatorname{Im} G$ it suffices to note that the entry $\frac{1}{2}\left(\alpha^{2}-\beta^{2}\right)+\sqrt{3} \alpha \beta=\frac{\alpha^{2}}{2}(1+\lambda(\sqrt{3}-\lambda))$ is positive whenever $0<\lambda<\sqrt{3}$. Noting that the Bergman kernel of $\mathcal{H}_{3}$ is of the form $c(\operatorname{det}(\operatorname{Im} \mathfrak{T}))^{-4}$ we have

$$
G^{*} \omega_{\mathcal{H}}=-4 \sqrt{-1} \partial \bar{\partial} \log (\operatorname{det}(\operatorname{Im} G(\tau)))=-4 \sqrt{-1} \partial \bar{\partial} \log (\operatorname{Im} \tau)=2 \omega_{\mathcal{H}},
$$

proving that $G:\left(\mathcal{H}, 2 d s_{\mathcal{H}}^{2}\right) \rightarrow\left(\mathcal{H}_{3}, d s_{\mathcal{H}_{3}}^{2}\right)$ is a holomorphic isometry, as desired.

Recall the cube-root map $\rho_{3}: \mathcal{H} \rightarrow \mathcal{H} \times \mathcal{H} \times \mathcal{H}$. Realizing the latter as a totally geodesic complex submanifold in $\mathcal{H}_{3}$ via a standard embedding $\iota: \mathcal{H} \times \mathcal{H} \times \mathcal{H} \rightarrow \mathcal{H}_{3}$ where the image consists precisely of all diagonal matrices in $\mathcal{H}_{3}$, we have a holomorphic isometry $F:=\iota \circ \rho_{3}:\left(\mathcal{H}, 2 d s_{\mathcal{H}}^{2}\right) \rightarrow\left(\mathcal{H}_{3}, d s_{\mathcal{H}_{3}}^{2}\right)$. Note that $\iota: \mathcal{H} \times \mathcal{H} \times \mathcal{H} \rightarrow \mathcal{H}_{3}$ is a holomorphic isometric embedding with respect to the Bergman metric with normalizing constant $\lambda=2$. For the holomorphic isometry $G: \mathcal{H} \rightarrow \mathcal{H}_{3}$, a priori it is not evident that $F$ and $G$ are incongruent to each other. They can however be distinguished by examining the nature of the branch points on $\partial \mathcal{H}_{3}$. More precisely, we have

Proposition 3.3.2. The two holomorphic isometric embeddings $F, G:\left(\mathcal{H}, 2 d s_{\mathcal{H}}^{2}\right) \rightarrow$ $\left(\mathcal{H}_{3}, d s_{\mathcal{H}_{3}}^{2}\right), F:=\iota \circ \rho_{3}$, are not congruent to each other. In fact, for any holomorphic isometric embedding $h: \mathcal{H} \rightarrow \mathcal{H} \times \mathcal{H} \times \mathcal{H}$, and for $H:=\iota \circ h$, the two holomorphic embeddings $G, H:\left(\mathcal{H}, 2 d s_{\mathcal{H}}^{2}\right) \rightarrow\left(\mathcal{H}_{3}, d s_{\mathcal{H}_{3}}^{2}\right)$ are incongruent to each other.

Proof. Regard $\mathcal{H} \times \mathcal{H} \times \mathcal{H}$ as an open subset of $\mathbb{P}^{1} \times \mathbb{P}^{1} \times \mathbb{P}^{1}$ and likewise the Siegel upper half-plane $\mathcal{H}_{3}$ canonically (via the Borel embedding) as an open subset of the compact dual $M$ of $\mathcal{H}_{3}$. The map $F: \mathcal{H} \rightarrow \mathcal{H}_{3}$ has two branch points on $\partial(\mathcal{H} \times \mathcal{H} \times \mathcal{H})$, viz. 0 and a point at infinity, both of which lie on the Shilov boundary of $\mathcal{H} \times \mathcal{H} \times \mathcal{H}$ and hence on the Shilov boundary $\operatorname{Sh}\left(\mathcal{H}_{3}\right)$ of $\mathcal{H}_{3}$. The branch point at infinity corresponds to the point 0 on the boundary of the image of $\widehat{F}:=-F(\tau)^{-1}$. Likewise the map $G: \mathcal{H} \rightarrow \mathcal{H}_{3}$ has two branch points on $\partial \mathcal{H}_{3}$, viz., the point $F(0)=\left[\begin{array}{lll}0 & 0 & 0 \\ 0 & i & 0 \\ 0 & 0 & 0\end{array}\right]$ and the branch point at infinity correspond to the branch point $\left[\begin{array}{lll}0 & 0 & 0 \\ 0 & i & 0 \\ 0 & 0 & 0\end{array}\right]$ of the map $\widehat{G}:=-G(\tau)^{-1}$. The finite part of $\operatorname{Sh}\left(\mathcal{H}_{3}\right)$ consists precisely of the real (symmetric) matrices lying on $\partial \mathcal{H}_{3}$. Thus the two branch points of $G$ on $\partial \mathcal{H}_{3}$ do not belong to the Shilov boundary, which implies that $F$ and $G$ are incongruent to each other.

For the general case of $H=\iota \circ h$ in place of $F$, according to [Ng, Theorem 8.1], the set of all holomorphic isometries $h: \mathcal{H} \rightarrow \mathcal{H} \times \mathcal{H} \times \mathcal{H}$ up to normalizing constants are completely determined. In particular, when the normalizing constant is $\lambda=1, h$ is either congruent to the cube-root map $\rho_{3}$, or to $(\rho(\sqrt{\tau}), i \sqrt{\tau})$, where $\rho: \mathcal{H} \rightarrow \mathcal{H} \times \mathcal{H}$ is a holomorphic map congruent to the square-root map $\rho_{2}$. The map $\rho$ is singular exactly at two distinct points $b_{1}, b_{2} \in \partial \mathcal{H} \cup\{\infty\}$. If $H=\iota \circ h$ and $G$ are congruent as maps from $\mathcal{H}$ to $\mathcal{H}_{3}$, then we must have $\left\{b_{1}, b_{2}\right\}=\{0, \infty\}$, and in this case $\rho=\psi \circ \mu$, where $\mu(\tau)=(\sqrt{\tau}, i \sqrt{\tau})$, and $\psi \in \operatorname{Aut}(\mathcal{H} \times \mathcal{H})$. In this case $H$ is congruent to the map $S(\tau)=\left(\tau^{1 / 4}, i \tau^{1 / 4}, i \tau^{1 / 2}\right)$. The latter $S$ has exactly two branch points, the point 0 and an infinite point corresponding to the branch point 0 of the map $\widehat{S}: \mathcal{H} \rightarrow \mathcal{H}_{3}$ defined 
by $\widehat{S}(\tau)=-S(\tau)^{-1}$. In particular, both branch points of $H$ lie on $\operatorname{Sh}\left(\mathcal{H}_{3}\right)$, implying that $G, H: \mathcal{H} \rightarrow \mathcal{H}_{3}$ are not congruent to each other.

\section{Bona fide holomorphic isometries between complete circular domains}

\subsection{From holomorphic isometries to norm-preserving extensions of square-integrable holomorphic functions}

In this section we explore the meaning of holomorphic isometries in a special case, viz., bona fide holomorphic isometries between bounded complete circular domains. Here a holomorphic mapping between two Bergman manifolds is said to be a bona fide isometry if it is an isometry with respect to the Bergman metric, i.e., the normalizing constant is $\lambda=1$. We will show that they lead to norm-preserving extensions of square-integrable functions which can be expressed explicitly in terms of the Bergman kernel.

For a Hilbert space $H$ we denote by $H^{*}$ its dual space. For any vector subspace $S \subset H$ we denote by $S^{\perp}$ the orthogonal complement of $S$ in $H$, and by $S^{\text {Ann }} \subset H^{*}$ the annihilator of $S$ consisting of continuous linear functionals on $H$ vanishing on $S$.

For a bounded Euclidean domain $G$, we write $\Psi_{G}: G \hookrightarrow \mathbb{P}\left(H^{2}(G)^{*}\right)$ for the canonical embedding on $G, G^{\natural} \subset \mathbb{P}\left(H^{2}(G)^{*}\right)$ for its image $\Psi_{G}(G)$, to be called the canonical image. For $z \in G$, we denote by $\widehat{z} \in H^{2}(G)^{*}$ the continuous linear function on $H^{2}(G)$ given by $\widehat{z}(f)=f(z)$ for any $f \in H^{2}(G)$. Fixing an orthonormal basis $\left(h_{i}\right)_{i=0}^{\infty}$ and denoting by $\mathbb{H}$ the Hilbert space of square-integrable sequences of complex numbers, we also write $\Phi_{G}(z)=\left(h_{0}(z), h_{1}(z), \ldots\right) \in \mathbb{H}$, and write $\Psi_{G}(z)=\left[\Phi_{G}(z)\right] \in \mathbb{P}(\mathbb{H})$.

Lemma 4.1.1. $G^{\natural} \subset \mathbb{P}\left(H^{2}(G)^{*}\right)$ is topologically linearly non-degenerate, i.e., denoting by $\operatorname{Span}\left(G^{\natural}\right) \subset \mathbb{P}\left(H^{2}(G)^{*}\right)$ the projective linear span of $G^{\natural}$, we have $\overline{\operatorname{Span}\left(G^{\natural}\right)}=$ $\mathbb{P}\left(H^{2}(G)^{*}\right)$ for its topological closure.

Proof. Let $\left(a_{0}, a_{1}, \ldots\right)$ be a square-integrable sequence of complex numbers orthogonal to the image of $\Phi_{G}$. Then, writing $h:=\overline{a_{0}} h_{0}+\overline{a_{1}} h_{1}+\cdots \in H^{2}(G)$ we have $h(z)=0$ for every $z \in G$, which is absurd unless $a_{i}=0$ for $0 \leq i<\infty$, as desired.

Let now $D \Subset \mathbb{C}^{n}$ and $\Omega \Subset \mathbb{C}^{N}$ be bounded complete circular domains. Suppose $F$ : $D \rightarrow \Omega$ is a bona fide holomorphic isometric embedding with respect to the Bergman metric. Identifying $D$, resp. $\Omega$, with its canonical image $D^{\natural} \subset \mathbb{P}\left(H^{2}(D)^{*}\right)$, resp. $\Omega^{\natural} \subset$ $\mathbb{P}\left(H^{2}(\Omega)^{*}\right), F: D \rightarrow \Omega$ corresponds to a holomorphic isometry $F^{\natural}: D^{\natural} \rightarrow \Omega^{\natural}$. Since $D^{\natural} \subset \mathbb{P}\left(H^{2}(D)^{*}\right)$ and $\Omega^{\natural} \subset \mathbb{P}\left(H^{2}(\Omega)^{*}\right)$ are topologically linearly non-degenerate, by Calabi [Ca], $F^{*}$ is induced by some linear isometry $\Theta: H^{2}(D)^{*} \rightarrow H^{2}(\Omega)^{*}$. Identifying a Hilbert space with its dual by a conjugate linear map, $\Theta$ is equivalently given by a linear isometry $\mu: H^{2}(D) \rightarrow H^{2}(\Omega)$ onto a Hilbert subspace. In the case at hand, we determine $\mu$ in terms of the Bergman kernels, as follows.

Theorem 4.1.1. Let $D \Subset \mathbb{C}^{n}$ and $\Omega \Subset \mathbb{C}^{N}$ be complete circular domains, and assume that $t D \subset D$ and $t \Omega \subset \Omega$ for $0<t<1$. Let $F:\left(D, d s_{D}^{2}\right) \rightarrow\left(\Omega, d s_{\Omega}^{2}\right)$ be a holomorphic isometric embedding with $F(0)=0$. Write $Z:=F(D) \subset \Omega$, and denote by $F^{-1}: Z \rightarrow D$ the inverse of $F: D \rightarrow Z$. Define $J:=\left\{g \in H^{2}(\Omega):\left.g\right|_{Z} \equiv 0\right\}$. Then, for 
the canonical embedding $\Psi_{\Omega}: \Omega \hookrightarrow \mathbb{P}\left(H^{2}(\Omega)^{*}\right)$, we have $\overline{\operatorname{Span}\left(\Psi_{\Omega}(Z)\right)}=\mathbb{P}\left(J^{\mathrm{Ann}}\right)$. Moreover, the holomorphic isometry $F$ is induced by a linear isometry $\mu: H^{2}(D) \rightarrow$ $H^{2}(\Omega)$ such that $\mu(s) \mid z=s \circ F^{-1}$ for any $s \in H^{2}(D)$ and $E:=\operatorname{Im} \mu=J^{\perp}$.

We write $K_{D, w}(z):=K_{D}(z, w)$ and $K_{\Omega, \xi}(\zeta):=K_{\Omega}(\zeta, \xi)$. For $J \subset H^{2}(\Omega)$ we have

Lemma 4.1.2. For the Hilbert subspace $J \subset H^{2}(\Omega)$ consisting of square-integrable holomorphic functions vanishing on $Z$, we have $J^{\perp}=\overline{\operatorname{Span}\left(\left\{K_{\Omega, \zeta}: \zeta \in Z\right\}\right)}$.

Proof. By the reproducing property of $K_{\Omega}$, we have $h(\zeta)=\int_{\Omega} K_{\Omega}(\zeta, \xi) h(\xi) d V(\xi)$ for any $h \in H^{2}(\Omega)$, where $d V$ denotes the Euclidean volume form. Thus, for any $\zeta \in \Omega$, $h(\zeta)=0$ whenever $h \perp K_{\Omega, \zeta}$, hence $h \in J$ whenever $h \perp K_{\Omega, \zeta}$ for every $\zeta \in Z$. It follows that $J^{\perp}$ is the minimal Hilbert subspace of $H^{2}(\Omega)$ containing $K_{\Omega, \zeta}$ for each $\zeta \in Z$, i.e., the topological closure of the linear span of $\left\{K_{\Omega, \zeta}: \zeta \in Z\right\}$, as desired.

Proof of Theorem 4.1.1. Without loss of generality we may assume that both $D \Subset \mathbb{C}^{n}$ and $\Omega \Subset \mathbb{C}^{N}$ are of Euclidean volume 1, so that $K_{D}(z, 0)=1$ for $z \in D$ and $K_{\Omega}(\zeta, 0)=1$ for $\zeta \in \Omega$. By Proposition 1.1.1, $K_{\Omega}(F(z), F(w))=K_{D}(z, w)$ for any $z, w \in D$. By the reproducing property of $K_{D}(z, w)$, for $s \in H^{2}(D)$ we have

$$
s(z)=\int_{D} K_{D}(z, w) s(w) d V(w),
$$

where $d V$ denotes the Euclidean volume form. For $0<t<1$ and $s \in H^{2}(D)$ define

$$
\mu_{t}(s)(\zeta)=\int_{D} K_{\Omega}(\zeta, F(t w)) s(w) d V(w),
$$

noting that for $0<t<1$ the right-hand side is well-defined since in fact $t D \Subset D$, so that $K_{\Omega}(\zeta, F(t w))$ is bounded as a function of $w \in D$, and we have $\mu_{t}(s) \in H^{2}(\Omega)$ since $\left\|K_{\Omega, F(t w)}\right\|_{H^{2}(\Omega)}$ is uniformly bounded for $w \in D$. On the other hand, the right-hand side of (1) is a priori undefined when $t=1$ since the holomorphic function $\varphi(w):=$ $K_{\Omega}(F(w), \zeta)$ is not known to be in $H^{2}(D)$. We are going to show nonetheless that, as $t \rightarrow 1^{-}, \mu_{t}: H^{2}(D) \rightarrow H^{2}(\Omega)$ converges weakly to some linear isometry $\mu$ : $H^{2}(D) \rightarrow H^{2}(\Omega)$. For $s \in H^{2}(D)$ and $0<t<1$, write $h_{t}=\mu_{t}(s)$. Then

$$
\begin{aligned}
\left\|h_{t}\right\|_{H^{2}(\Omega)}^{2} & \\
& =\int_{\Omega}\left(\int_{D} K_{\Omega}\left(\zeta, F\left(t w^{\prime}\right)\right) s\left(w^{\prime}\right) d V\left(w^{\prime}\right)\right)\left(\overline{\int_{D} K_{\Omega}(\zeta, F(t w)) s(w) d V(w)}\right) d V(\zeta) \\
& =\int_{D}\left(\int_{D}\left(\int_{\Omega} K_{\Omega}(F(t w), \zeta) K_{\Omega}\left(\zeta, F\left(t w^{\prime}\right)\right) d V(\zeta)\right) s\left(w^{\prime}\right) d V\left(w^{\prime}\right)\right) \overline{s(w)} d V(w) .
\end{aligned}
$$

Since $t \Omega \subset \Omega$, by Lemma 1.1.1 it follows that $K_{\Omega}(\zeta, \xi)$ extends holomorphically in $(\zeta, \bar{\xi})$ to some neighborhood of $\bar{\Omega} \times t \Omega$. Hence $K_{\Omega}(\zeta, F(t w))$ is uniformly bounded on 
$\Omega \times D$, which justifies the change of order of integration by Fubini's theorem in (2). By the reproducing property of $K_{\Omega}(\zeta, \xi)$ applied to $\left.\theta(\zeta):=K_{\Omega}\left(\zeta, F\left(t w^{\prime}\right)\right)\right)$ on $\Omega$, we have

$$
\begin{aligned}
\int_{\Omega} K_{\Omega}(F(t w), \zeta) K_{\Omega}\left(\zeta, F\left(t w^{\prime}\right)\right) d V(\zeta) & =\int_{\Omega} K_{\Omega}(F(t w), \zeta) \theta(\zeta) d V(\zeta) \\
& =\theta(F(t w))=K_{\Omega}\left(F(t w), F\left(t w^{\prime}\right)\right)
\end{aligned}
$$

Thus,

$$
\begin{aligned}
\int_{\Omega}\left|h_{t}(\zeta)\right|^{2} d V(\zeta) & =\int_{D}\left(\int_{D} K_{\Omega}\left(F(t w), F\left(t w^{\prime}\right)\right) s\left(w^{\prime}\right) d V\left(w^{\prime}\right)\right) \overline{s(w)} d V(w) \\
& =\int_{D}\left(\int_{D} K_{D}\left(t w, t w^{\prime}\right) s\left(w^{\prime}\right) d V\left(w^{\prime}\right)\right) \overline{s(w)} d V(w) \\
& =\int_{D}\left(\int_{D} K_{D}\left(t^{2} w, w^{\prime}\right) s\left(w^{\prime}\right) d V\left(w^{\prime}\right)\right) \overline{s(w)} d V(w) \\
& =\int_{D} s\left(t^{2} w\right) \overline{s(w)} d V(w) .
\end{aligned}
$$

By exactly the same arguments, for $0<t_{1}, t_{2}<1$ we have

$$
\begin{aligned}
\left\langle\mu_{t_{1}}(s), \overline{\mu_{t_{2}}(s)}\right\rangle_{H^{2}(\Omega)} & =\int_{\Omega} h_{t_{1}}(\zeta) \overline{h_{t_{2}}(\zeta)} d V(\zeta)=\int_{D} s\left(t_{1} t_{2} w\right) \overline{s(w)} d V(w), \\
\left\|\mu_{t_{1}}(s)-\mu_{t_{2}}(s)\right\|_{H^{2}(\Omega)}^{2} & =\int_{D}\left(s\left(t_{1}^{2} w\right)+s\left(t_{2}^{2} w\right)-2 s\left(t_{1} t_{2} w\right)\right) \overline{s(w)} d V(w) .
\end{aligned}
$$

As $t_{1}, t_{2} \rightarrow 1^{-}$, the function $\delta_{t_{1}, t_{2}}(s): s\left(t_{1}^{2} w\right)+s\left(t_{2}^{2} w\right)-2 s\left(t_{1} t_{2} w\right)$ tends to 0 in $H^{2}(D)$, hence $\left\|\mu_{t_{1}}(s)-\mu_{t_{2}}(s)\right\|_{H^{2}(\Omega)}$ converges to 0 . As a consequence, the weak limit $\mu$ of $\mu_{t}$ : $H^{2}(D) \rightarrow H^{2}(\Omega)$ exists. By (3), $\|\mu(s)\|_{H^{2}(\Omega)}=\|s\|_{H^{2}(D)}$, i.e., $\mu: H^{2}(D) \rightarrow H^{2}(\Omega)$ is a Hilbert space isomorphism onto some Hilbert subspace $E \subset H^{2}(\Omega)$, which we proceed to identify. From the definition of $\mu_{t}$ for $0<t<1$ in $(2), \mu_{t}(s)$ is a limit in $H^{2}(\Omega)$ of linear combinations of $K_{\Omega, F(t w)}$ as $w$ ranges over $D$. Now $f \in H^{2}(\Omega)$ is orthogonal to $\operatorname{Im} \mu_{t}=: E_{t} \subset H^{2}(\Omega)$ whenever it vanishes at every point of $F(t w), w \in D$ (cf. proof of Lemma 4.1.2). Since $J=\left\{f \in H^{2}(\Omega):\left.f\right|_{Z} \equiv 0\right\}$, for $0<t<1$ we have $J \subset E_{t}^{\perp}$, and hence $J \subset E^{\perp}$ when one passes to the limit as $t \rightarrow 1^{-}$, i.e., $E \subset J^{\perp}$. From (1) and the reproducing property of $K_{D}(z, w)$, for $\zeta \in \Omega$ and $w \in D$ we have

$$
\begin{aligned}
\mu_{t}\left(K_{D, w}\right)(\zeta) & =\overline{\left.\int_{D} K_{D}\left(w, w^{\prime}\right) K_{\Omega}\left(F\left(t w^{\prime}\right), \zeta\right)\right) d V\left(w^{\prime}\right)} \\
& =\overline{K_{\Omega}(F(t w), \zeta)}=K_{\Omega}(\zeta, F(t w))=K_{\Omega, F(t w)}(\zeta)
\end{aligned}
$$

Hence,

$$
\mu\left(K_{D, w}\right)=\lim _{t \rightarrow 1^{-}} \mu_{t}\left(K_{D, w}\right)=\lim _{t \rightarrow 1^{-}} K_{\Omega, F(t w)}=K_{\Omega, F(w)}
$$

As a result, $E$ contains $\overline{\operatorname{Span}\left(\left\{K_{\Omega, \zeta}: \zeta \in Z\right\}\right)}$, which is precisely $J^{\perp}$. Thus, $E \supset J^{\perp}$ and hence $E=J^{\perp}$. Recall that for $z \in D, \widehat{z} \in H^{2}(D)^{*}$ is identified with $\Phi_{D}(z)$, and 
similarly for $\zeta \in \Omega, \widehat{\zeta} \in H^{2}(\Omega)^{*}$ is identified with $\Phi_{\Omega}(\zeta)$. Denoting by $v: E \rightarrow H^{2}(D)$ the inverse isomorphism of $\mu: H^{2}(D) \rightarrow E$, for the adjoint operator $\nu^{*}: H^{2}(D)^{*} \rightarrow E^{*}$ we have

$$
v^{*}(\widehat{z})\left(K_{\Omega, F(w)}\right)=\widehat{z}\left(K_{D, w}\right)=K_{D}(z, w)=K_{\Omega}(F(z), F(w))=\widehat{F(z)}\left(K_{\Omega, F(w)}\right),
$$

which gives $\Theta: H^{2}(D)^{*} \rightarrow H^{2}(\Omega)^{*}$ inducing the holomorphic isometry $F^{\natural}: D^{\natural} \rightarrow \Omega^{\natural}$ when we define $\Theta(\lambda)(f)=0$ for any $\lambda \in H^{2}(D)^{*}$ and $f \in J$. Then $\overline{\Theta\left(H^{2}(D)^{*}\right)}$ is precisely $J^{\text {Ann }}$, and $\overline{\operatorname{Span}\left(F^{\natural}\left(D^{\natural}\right)\right)}=\mathbb{P}\left(\Theta\left(H^{2}(D)^{*}\right)\right)=\mathbb{P}\left(J^{\text {Ann }}\right)$. Finally, for $0<t<1$,

$$
\begin{aligned}
\mu_{t}(s)(F(z)) & =\int_{D} K_{\Omega}(F(z), F(t w)) s(w) d V(w)=\int_{D} K_{D}(z, t w) s(w) d V(w) \\
& =\int_{D} K_{D}(t z, w) s(w) d V(w)=s(t z), \\
\mu(s)(F(z)) & =\lim _{t \rightarrow 1^{-}} \mu_{t}(s)(F(z))=\lim _{t \rightarrow 1^{-}} s(t z)=s(z),
\end{aligned}
$$

hence $\left.\mu(s)\right|_{Z}=s \circ F^{-1}$, completing the proof of Theorem 4.1.1.

Acknowledgments. The author would like to thank Laurent Clozel for posing to him the circle of differential-geometric problems arising from his work with Ullmo on modular correspondences. He would like to thank Sui-Chung $\mathrm{Ng}$ for discussions concerning examples of holomorphic isometries, especially for the simplification of an example of such a map from the upper half-plane to a Siegel upper half-plane explained here in (3.3), and for helpful observations and comments on the article. $\mathrm{He}$ also wishes to thank the referees for many helpful comments for the improvement of the article. The research work leading to the current article was partially supported by the GRF grant 7018/03 of Hong Kong Research Grants Council.

\section{References}

[Al] Alexander, H.: Holomorphic mappings from the ball and the polydisc. Math. Ann. 209, 249-256 (1974) Zbl 0272.32006 MR 0352531

[Ca] Calabi, E.: Isometric imbedding of complex manifolds. Ann. of Math. 58, 1-23 (1953) Zbl 0051.13103 MR 0057000

[CU] Clozel, L., Ullmo, E.: Modular correspondences and invariant measures. J. Reine Angew. Math. 558, 47-83 (2003) Zbl 1042.11027

[FK] Faraut, J., Korányi, A.: Function spaces and reproducing kernels on bounded symmetric domains. J. Funct. Anal. 88, 64-89 (1990) Zbl 0718.32026 MR 1033914

[He] Helgason, S.: Differential Geometry, Lie Groups, and Symmetric Spaces. Academic Press, Orlando (1978) Zbl 0451.53038 MR 0514561

[Ih] Ihara, S.: Holomorphic embeddings of symmetric domains. J. Math. Soc. Japan. 19, 261302 (1967); Supplement, ibid., 543-544 Zbl 0159.11201 MR 0222335

[Mk1] Mok, N.: Uniqueness theorems of Hermitian metrics of seminegative curvature on locally symmetric spaces of negative Ricci curvature. Ann. of Math. 125, 105-152 (1987) Zbl 0616.53040 MR 0873379

[Mk2] Mok, N.: Metric Rigidity Theorems on Hermitian Locally Symmetric Manifolds. Ser. Pure Math. 6, World Sci. (1989) Zbl 0912.32026 MR 1081948 
[Mk3] Mok, N.: Local holomorphic isometric embeddings arising from correspondences in the rank-1 case. In: Contemporary Trends in Algebraic Geometry and Algebraic Topology, S.-S. Chern et al. (eds), World Sci., 155-166 (2002) Z Zbl 1083.32019 MR 1945359

[Mk4] Mok, N.: On the asymptotic behavior of holomorphic isometries of the Poincaré disk into bounded symmetric domains. In: Special Volume in honor of Prof. Wu Wenjun on the occasion of his 90th birthday, Acta Math. Sci. 29B, 881-902 (2009) Zbl 1212.53027 MR 2509997

[Mk5] Mok, N.: Geometry of holomorphic isometries and related maps between bounded domains. In: Geometry and Analysis, Vol. II, Adv. Lect. Math. 18, Higher Education Press and Int. Press, Beijing-Boston, 225-270 (2011) MR 2882446

[Ng] Ng, S.-C.: On holomorphic isometric embeddings of the unit disk into polydisks. Proc. Amer. Math. Soc. 138, 2907-2922 (2010) Zbl 1207.32018 MR 2644903

[Sa] Satake, I.: Holomorphic embeddings of symmetric domains into a Siegel space. Amer. J. Math. 90, 425-461 (1965) Zbl 0144.08202 MR 0196134

[Wo] Wolf, J. A.: Fine structure of Hermitian symmetric spaces. In: Symmetric Spaces, W. M. Boothby and G. L. Weiss (eds.), Dekker, New York, 271-357 (1972) Zbl 0257.32014 MR 0404716 